\title{
Endocytic vacuole formation by WASH-mediated endocytosis
}

2

3 Pia Brinkert (1), Lena Krebs (1), Pilar Samperio Ventayol (1), Lilo Greune (2), Carina

4 Bannach (1), Delia Bucher (3), Jana Kollasser (5), Petra Dersch (2), Steeve Boulant

$5 \quad(3,4)$, Theresia E.B. Stradal $(5)$, Mario Schelhaas $\left(1,6,{ }^{*}\right)$

6

7 (1) Institute of Cellular Virology, ZMBE, University of Münster, 48149 Münster, Germany

8 (2) Institute of Infectiology, ZMBE, University of Münster, 48149 Münster, Germany

9 (3) Department of Infectious Diseases, Virology, Heidelberg University Hospital, 69120 Heidelberg, Germany

10 (4) German Cancer Research Center (DKFZ), 69120 Heidelberg, Germany

11 (5) Department of Cell Biology, Helmholtz Centre for Infection Research, 38124 Braunschweig, Germany

12 (6) Cells in Motion Interfaculty Center, University of Münster, 48149 Münster, Germany

13 * Lead contact: schelhaas@uni-muenster.de

14

15

16 


\section{Summary}

18 Endocytosis of extracellular or plasma membrane material is a fundamental process.

19 A variety of endocytic pathways exist, several of which are barely understood in 20 terms of mechanistic execution and biological function. Importantly, some 21 mechanisms have been identified and characterized by following virus internalization 22 into cells. This includes a novel endocytic pathway exploited by human 23 papillomavirus type 16 (HPV16). However, its cellular role and mechanism of 24 endocytic vacuole formation remain unclear. Here, HPV16 was used as a tool to 25 examine the mechanistic execution of vesicle formation by combining systematic 26 perturbation of cellular processes with electron and video microscopy. Our results 27 indicate cargo uptake by uncoated, inward-budding pits facilitated by the membrane 28 bending retromer protein SNX2. Actin polymerization-driven vesicle scission is 29 promoted by $\mathrm{WASH}$, an actin regulator typically not found at the plasma membrane.

30 Uncovering a novel role of WASH in endocytosis, we propose to term the new 31 pathway WASH-mediated endocytosis (WASH-ME). 


\section{Introduction}

34 Cells internalize extracellular ligands, fluids, plasma membrane lipids and receptors

35 by endocytosis. This is crucial for cellular and organismal homeostasis, signal

36 transduction, intercellular communication, immune response, development and

37 neurotransmission (Schmid and Conner, 2003). Several endocytic mechanisms exist

38 that are distinguished by diverse criteria. Morphologically, cargo is engulfed either by

39 outward membrane protrusions (macropinocytosis, phagocytosis) or by inward

40 budding pits that may or may not display a visible coat in electron microscopy (EM)

41 (e.g. clathrin-mediated endocytosis (CME), glycosphingolipid enriched endocytic

42 carriers (GEEC)) (Heuser and Evans, 1980; Kirkham et al., 2005). The cellular

43 machinery executing endocytic vacuole formation and the destination of endocytic

44 cargo further define the identity of endocytic pathways (Doherty and McMahon,

45 2009). For instance, macropinocytosis occurs by growth factor-induced, actin-driven

46 protrusions that form large vacuoles upon backfolding with help of the

47 Bin1/Amphiphysin/Rvs (BAR) protein C-terminal binding protein 1 (CtBP1) (Liberali et

48 al., 2008; Lim and Gleeson, 2011). Of the inward budding mechanisms, CME occurs

49 by sequential recruitment of adaptor proteins (AP) such as AP2 and the clathrin coat.

50 Dynamin-2 mediated scission then leads to vacuole formation (McMahon and

51 Boucrot, 2011; Merrifield and Kaksonen, 2014). In contrast, caveolar endocytosis

52 occurs by preassembled coat structures (Pelkmans et al., 2004; Hayer et al., 2010;

53 Stoeber et al., 2016). Another criterium is cargo specificity. For instance,

54 macropinocytosis and CME are responsible for bulk fluid uptake or specific

55 membrane receptor internalization, respectively. A subset of GEEC is specifically

56 responsible for lectin uptake (Lakshminarayan et al., 2014). In addition to the well-

57 established pathways, a number of less well understood endocytic mechanisms have

58 emerged. Only little information is available on which cellular processes they 
59 regulate, or on how vesicles are formed, as they are defined by their independence

60 of classical endocytic regulators such as clathrin, caveolin, dynamin-2, or cholesterol

61 (Doherty and McMahon, 2009). Importantly, several of these mechanisms have been

62 identified by following the internalization of viruses such as lymphocytic

63 choriomeningitis virus (LCMV), influenza A virus (IAV), and human papillomavirus

64 type 16 (HPV16) (Rust et al., 2004; Quirin et al., 2008; Mercer, Schelhaas and

65 Helenius, 2010; Schelhaas et al., 2012).

66

67 As intracellular parasites, viruses depend on host cells for their life-cycle. This is 68 particularly important during the initial phase of infection termed entry, during which 69 viruses deliver their genome to the site of replication within cells. As virus particles 70 lack locomotive abilities, they strictly rely on cellular transport mechanisms to cross 71 the plasma membrane, the cytosol, or the nuclear envelope. Following viral particles 72 en route to the site of replication thus allows to study cellular mechanisms exploited 73 by viruses (Marsh and Helenius, 2006). Most viruses make use of endocytosis to 74 overcome the plasma membrane. Among them is HPV16, a small non-enveloped 75 DNA virus and the leading cause of cervical cancer (de Sanjose et al., 2010; 76 Mirabello et al., 2017). HPV16 initially infects basal keratinocytes of squamous 77 mucosal epithelia, whereas completion of its life cycle requires suprabasal 78 keratinocyte differentiation (Doorbar, 2005). For entry, it utilizes a unique endocytic 79 pathway for which little to no mechanistic information exists (Schelhaas et al., 2012).

80 As viruses strictly hijack existing endocytic mechanisms, the intriguing question 81 remains which cellular cargo targets this pathway. Uptake of HPV16 into cells is slow 82 and asynchronous, with individual virus internalization events occurring over a period 83 of many hours after binding (Schelhaas et al., 2012; Becker et al., 2018). Binding to 84 heparan sulfate proteoglycans (HSPGs) initiates crucial structural changes in the 
85 virus capsid that allow transfer to an internalization receptor (complex) to induce 86 uptake (Giroglou et al., 2001; Richards et al., 2006; Selinka et al., 2007; Cerqueira et 87 al., 2013, 2015; Becker et al., 2018). Uptake involves growth factor receptors 88 (Schelhaas et al., 2012; Surviladze, Dziduszko and Ozbun, 2012; Bannach et al., 89 2020), integrin $\alpha 6$ (Evander et al., 1997; Yoon et al., 2001; Scheffer et al., 2013), 90 tetraspanins cluster of differentiation (CD) 151 and CD63 (Scheffer et al., 2013; Fast 91 et al., 2018), and annexin A2 heterotetramer (Woodham et al., 2012; Dziduszko and 92 Ozbun, 2013). These proteins are thought to constitute specifically functionalized tetraspanin-enriched microdomains termed HPV16 entry platforms that induce 94 uptake and co-internalize with the virus (Raff et al., 2013; Florin and Lang, 2018). 95 After endocytosis, HPV16 traffics through the endosomal system, accompanied by 96 partial capsid disassembly (Smith et al., 2008; Spoden et al., 2008; Bienkowska97 Haba et al., 2012). From endosomes, the virus is routed to the trans-Golgi network 98 by retrograde transport (Day et al., 2013; Lipovsky et al., 2013) and reaches its site 99 of replication, the nucleus, after nuclear envelope breakdown during mitosis (Pyeon 100 et al., 2009; Aydin et al., 2014).

102 HPV16 exploits an endocytic pathway independent of a long list of prominent 103 endocytic regulators including clathrin, caveolin, dynamin and cholesterol. Instead, it 104 depends on sodium/proton exchange, actin polymerization and signaling via epidermal growth factor receptor (EGFR), phosphatidylinositol-4,5-bisphosphate 3-kinase, p21-activated kinase 1, protein kinase C, and Abelson tyrosine-protein

107 kinase 2 (Abl2) (Schelhaas et al., 2012; Bannach et al., 2020). It remains largely 108 elusive how these factors contribute to endocytic vesicle formation. We know, 109 however, that EGFR and Abl2 regulate endocytosis induction and pit maturation, 110 respectively (Bannach et al., 2020). As such, the requirements for HPV16 uptake are 
111 somewhat reminiscent of macropinocytosis, during which sodium/proton exchange

112 regulates large actin-driven membrane protrusions to engulf bulk extracellular

113 material (Doherty and McMahon, 2009; Mercer, Schelhaas and Helenius, 2010).

114 However, HPV16 endocytosis typically involves small vesicles of about 60-100 nm

115 diameter that contain single virus particles, and that are generated independently of

116 cholesterol and the classical Rho-like GTPases cell division cycle 42 (Cdc42), rat

117 sarcoma (Ras)-related C3 botulinum toxin substrate 1 (Rac1), and Ras homolog

118 family member 1 (RhoA) (Schelhaas et al., 2012; Bannach et al., 2020). Perhaps, it

119 represents a small-scale version of macropinocytosis, i.e. "micropinocytosis"

120 (Helenius, 2018, 2020).

122 Here, we used HPV16 as cargo of this unique endocytic pathway to address how

123 vesicles would be mechanistically formed. We demonstrated that HPV16

124 endocytosis occurred via uncoated vesicles severed from the plasma membrane by

125 actin-related proteins (Arp) 2/3 complex-dependent branched actin polymerization.

126 Interestingly, Arp2/3 complex activation did not involve nucleation promoting factors

127 (NPFs) typically found at the plasma membrane, but the Wiskott-Aldrich syndrome 128 protein (WASP) and suppressor of cAMP receptor (Scar) homologue (WASH), a well-

129 known regulator of endosomal cargo sorting. WASH was recruited to endocytic sites

130 at the same time as sorting nexin 2 (SNX2), a BAR domain protein of the endosomal

131 retromer complex, which supposedly assists in membrane bending during endocytic

132 vesicle formation. Providing insights into the mechanism of a unique endocytic

133 pathway, our findings for the first time conclusively demonstrated a direct

134 involvement of WASH in endocytosis and implicated retromer components in 135 endocytic cargo uptake. 


\section{Results}

138 HPV16 endocytosis occurred via uncoated, inward budding vesicles

139 To assess how this unique mechanism generates endocytic vesicles, we employed

140 HPV16 as trackable cargo in correlative light and EM (CLEM). Our goal was to

141 visualize different stages of endocytic vacuole formation and to address whether

142 potential coat structures were associated with these vacuoles, similar to clathrin-

143 coated pits or caveolae. For this, fluorescent virus particles were correlated with

144 structures on the cytosolic leaflet of plasma membrane sheets analyzed by

145 transmission EM (TEM) of metal replicas (Heuser and Evans, 1980; Sochacki et al.,

146 2014; Bucher et al., 2018). As expected, different stages of clathrin-coated pit

147 formation were readily observable at sites distinct from virus localizations (Figure

148 1A). For HPV16, about $20 \%$ of viruses were associated with no obvious structure,

149 likely representing virus binding to HSPGs prior to engagement of HPV16 entry

150 platforms and endocytosis induction. A considerable proportion (31\%) was correlated

151 with dense actin network patches (Figure 1B). These may constitute anchoring

152 structures for tetraspanin microdomains/HPV entry platforms, either prior to or during

153 induction of endocytosis (Schelhaas et al., 2008; Ménager and Littman, 2016).

154 Importantly, inward-budding structures at virus sites were readily observed and

155 classified into stages of vesicle formation in analogy to CME (Figures 1A and 1B).

156 Designated as early stage, $14 \%$ of virions were associated with roughened plasma

157 membrane patches representing initial curvature formation (Figures $1 \mathrm{~A}$ and 1B).

158 Small invaginations (100-150 $\mathrm{nm}$ in diameter) were assigned as intermediate stage,

159 and represented pit maturation (13\%). Fully rounded invaginations of $80-100 \mathrm{~nm}$

160 (22\%) were classified as late-stage endocytic pits ready for scission (Figures $1 \mathrm{~A}$ and

161 1B). Notably, all invaginations were devoid of a discernable coat or other regular

162 structures (Figure 1A). Thus, HPV16 endocytosis occurred by a stepwise, inward 
163 budding process, in which endocytic vacuoles were formed without observable

164 contribution of a coat structure.

165

166 Actin polymerization facilitates HPV16 endocytosis (Schelhaas et al., 2012).

167 Accordingly, perturbation of actin assembly by cytochalasin $D$ (cytoD) reduced

168 HPV16 infection (Figure 1C). To analyze how defective actin assembly impacts the

169 steps of endocytic pit formation, we used CLEM of cytoD-treated cells. As expected,

170 the actin network population was no longer detectable (Figure 1B). However, notably

171 all stages of membrane invaginations were present to a similar extend in cytoD- and

172 untreated samples (Figure 1B). This indicated not only that pit formation was

173 independent of actin polymerization, but also that actin polymerization was

174 expendable for the induction of endocytic pits responsible for HPV16 uptake. In

175 addition, virus-correlated tubular structures devoid of an apparent coat newly

176 appeared in cytoD-treated cells (Figure 1B). In fact, these tubular membrane

177 invaginations were filled with virus particles (Figure 1D). This suggested a fully

178 functional formation of endocytic structures, but a failure in vesicle scission from the

179 plasma membrane. In conclusion, scission but not induction and membrane

180 invagination were facilitated by polymerized or polymerizing actin filaments during

181 HPV16 endocytosis.

182

\section{Actin polymerization coincided with HPV16 endocytosis}

184 Consistent with actin dynamics aiding vesicle scission in HPV16 uptake, we 185 observed actin signals at the neck of constricted endocytic pits in immunogold 186 labelling EM (Figure 2A). To gain insights into how the dynamics of actin at virus 187 entry sites may facilitate vesicle scission, we employed live cell TIRF microscopy 188 (TIRF-M). In TIRF-M, only the basal plasma membrane is observable. Hence, HPV16 
189 endocytosis into the cell interior was denoted by a rapid loss of virus signal (Figures

190 2B and 2C, Suppl. Movie 1). Virus signal loss correlated with an increase of

191 filamentous actin signals (Figures 2B and 2C, Suppl. Movie 1) indicating actin

192 polymerization at the time of scission. Detailed analysis of intensity profiles revealed

193 that virus signal loss occurred for about $4 \mathrm{~s}$ (Figures 2C and 2D, Suppl. Movie 1).

194 The onset of actin polymerization was somewhat variable. However, the increase in

195 actin signals was on average initiated $7 \mathrm{~s}$ prior to virus internalization, peaked just

196 before virus uptake, and decreased thereafter (Figures 2C and 2D, Suppl. Movie 1).

197 The dynamics of actin polymerization resembled dynamin recruitment during scission

198 in CME, starting about $20 \mathrm{~s}$ prior to loss of the clathrin signal from the plasma

199 membrane (Figures S1A-S1C, Suppl. Movie 2) (Merrifield et al., 2002). Since

200 dynamin is dispensable for HPV16 endocytosis (Spoden et al., 2008; Schelhaas et

201 al., 2012), similar recruitment profiles suggested that actin functionally replaced

202 dynamin as a scission factor. Moreover, the dynamics of polymerization indicated

203 that polymerized actin did not merely serve as anchor for other scission factors, but

204 was specifically induced for and actively contributed to endocytic vesicle scission as

205 reported for other endocytic mechanisms (Merrifield et al., 2002; Pelkmans, Püntener

206 and Helenius, 2002; Mooren, Galletta and Cooper, 2012).

\section{Branched actin polymerization regulated HPV16 endocytosis}

209 To understand the mechanism of actin polymerization for HPV16 endocytosis, we

210 investigated the involvement of branched versus unbranched actin filaments. Formin-

211 mediated unbranched actin polymerization was perturbed by SMIFH2, a small

212 molecule inhibitor binding the formin homology 2 domain in formins (Rizvi et al.,

213 2009). SMIFH2 treatment dose dependently decreased vesicular stomatitis virus

214 (VSV) infection, confirming the role of formins in actin-dependent CME of VSV 
215 (Figure 3A) (Cureton et al., 2009). In contrast, SMIFH2 did not affect HPV16 infection

216 (Figure 3A). Furthermore, HPV16 infection was largely unaffected by siRNA-

217 mediated depletion of individual formins (Figure S2). However, sequestering the

218 Arp2/3 complex as key regulator of branched actin polymerization by overexpression

219 of the Arp2/3 binding domain of WASP and WASP-family verprolin-homologous

220 protein (WAVE) (Scar-WA) (Machesky and Insall, 1998) strongly reduced HPV16

221 infection compared to cells expressing a control lacking the Arp2/3 binding domain

222 (Scar-W) (Figure 3B). In line with a requirement of branched actin polymerization,

223 RNA interference (RNAi) with Arp3 expression reduced infection by about $80 \%$

224 (Figure 3C) compared to cells transfected with a non-targeting control (ctrl.).

225 Similarly, vaccinia virus (VV) infection by macropinocytosis (Mercer and Helenius,

226 2008; Mazzon and Mercer, 2014) was reduced upon Arp3 depletion, whereas Semliki

227 Forest virus (SFV) uptake by actin-independent CME (Marsh and Helenius, 1980;

228 Marsh, Kielian and Helenius, 1984; DeTulleo and Kirchhausen, 1998) was even

229 increased (Figure 3C). Taken together, active branched but not unbranched actin

230 polymerization was crucial for HPV16 infection.

232 To verify the impact on HPV16 uptake, we employed a bona fide virus endocytosis 233 assay. For this, HPV16 covalently labelled with the $\mathrm{pH}$-sensitive dye pHrodo was 234 used, which gives rise to fluorescence only upon delivery to acidic endosomal 235 organelles (Figure 3D) (Ventayol and Schelhaas, 2015; Becker et al., 2018).

236 Depletion of Arp3 reduced virus internalization by about 70\% (Figure 3D). Thus, 237 branched actin polymerization was essential for HPV16 endocytosis. 
240 Since the Arp2/3 complex is inherently inactive, how is it activated for virus uptake?

241 Several NPFs with distinct cellular functions and localizations regulate Arp2/3

242 complex activity (Stradal and Scita, 2006; Rottner, Hänisch and Campellone, 2010).

243 To identify, which NPF acted during HPV16 infection, we employed RNAi. Depletion

244 of the most prominent actin regulators in endocytic processes, neuronal WASP (N-

245 WASP) and WAVE isoforms 1 or 2, (Qualmann and Kelly, 2000; Suetsugu et al.,

246 2003; Chadda et al., 2007), did not alter HPV16 infection (Figures S3A-S3C and

247 S3F). N-WASP RNAi results were confirmed in cells stably expressing a short hairpin

248 RNA (data not shown). Taken together, N-WASP, WAVE1 and 2 were dispensable

249 for HPV16 uptake. Consequently, a potential involvement of NPFs typically not found

250 at the plasma membrane was assessed. Neither depletion of junction mediating and

251 regulatory protein, p53 cofactor (JMY), a regulator of DNA damage response and cell

252 migration (Shikama et al., 1999; Zuchero et al., 2009), nor depletion of WASP

253 homolog associated with actin, Golgi membranes and microtubules (WHAMM), which

254 is involved in the secretory pathway (Campellone et al., 2008), impaired infection with

255 HPV16 (Figures S3D-S3F). However, depletion of WASH, a well-known actin

256 regulator of endosomal cargo sorting (Linardopoulou et al., 2007; Derivery et al.,

257 2009; Duleh and Welch, 2010), strongly reduced HPV16 infection, whereas VV

258 uptake by macropinocytosis was only mildly affected (Figures 4A and S3F). In

259 conclusion, WASH was the major NPF during HPV16 infection rather than NPFs

260 typically found at the plasma membrane.

261

\section{WASH acted during late stages of HPV16 endocytic vesicle formation}

263 To date, no evidence exists that WASH facilitates endocytic vesicle formation.

264 However, since it was the only NPF important for HPV16 infection, we hypothesized

265 a novel role of WASH in regulating actin polymerization for endocytic vacuole 
266 formation. Consistently, silencing of WASH resulted in a strong decrease of HPV16

267 uptake (Figure 4B). In corroboration, HPV16 infection was completely abrogated in

268 CRISPR/Cas9-derived mouse fibroblast WASH knock-out (KO) cells (NIH-3T3,

269 Figures 4C and S4C). Ectopic expression of wild type WASH rescued HPV16

270 infection in these cells (Figure 4C and S4A), whereas expression of WASH lacking

271 the WASP-homology 2, central and acidic (WCA) domain crucial for Arp2/3 complex

272 activation did not (Figure S4A). This indicated that WASH was an essential actin

273 regulator during HPV16 uptake. In support, HPV16 infection was also strongly

274 reduced in human osteosarcoma WASH KO cells (U2OS) (Figures S4B and S4C),

275 which not only supported the importance of WASH for HPV16 infection, but also

276 suggested the functional existence of WASH-dependent endocytosis in cells derived

277 from different tissues and species.

279 After confirming that HPV16 binding to cells was unaffected in WASH KO cells 280 (Figures S4D and S4E), we investigated how loss of WASH would interfere with 281 HPV16 endocytosis by analyzing the morphology of virus-containing pits in ultra-thin 282 section TEM. Compared to wild type cells, the endocytic pits were morphologically 283 unaltered in WASH KO cells, i.e., they were fully formed and partially constricted at 284 the neck (Figure 4D). Thus, early stages of endocytic vesicle formation such as 285 induction and membrane invagination were independent of WASH. Strikingly, the 286 average number of virus containing plasma membrane invaginations more than 287 doubled in the absence of WASH (Figures 4D). Hence, HPV16 endocytosis was 288 stalled at a late stage in WASH KO cells. Taken together, the roles of actin 289 polymerization in vesicle scission, of WASH as the single NPF to promote actin 290 polymerization, and the loss of WASH stalling HPV16 endocytosis at a late stage of 
291 vesicle formation strongly suggested that WASH regulated endocytic vesicle scission

292 but not endocytic pit formation.

293

294 To directly stimulate vesicle scission, WASH would have to be present at virus 295 endocytosis sites. To probe recruitment of WASH to HPV16 entry platforms marked 296 by HA-CD151 (Scheffer et al., 2013), we employed the proximity ligation assay (PLA)

297 (Söderberg et al., 2006). A small fraction of virus particles co-localized with the PLA 298 signal of WASH and CD151 (Figure S5), indicating that WASH indeed acted at virus 299 entry sites. A limited association of HPV16 with WASH/CD151 structures was 300 expected due to the highly asynchronous virus uptake over many hours, during which 301 only few viruses interact with entry platforms at any given time (Schelhaas et al., 302 2012; Becker et al., 2018). To more conclusively demonstrate recruitment of WASH 303 to virus entry sites and to gain information on recruitment dynamics, WASH 304 association in relation to uptake of HPV16 was analyzed by live cell TIRF-M. WASH 305 was detected together with virus signals for more than 50 seconds prior to HPV16 306 uptake and co-internalized with the virus (Figures 4E and 4F, Suppl. Movie 3). Thus,

307 WASH was recruited already early during vesicle formation, although it likely exerted 308 its NPF function only during scission (Figure 4D). To further investigate the stage of 309 WASH recruitment to virus endocytic sites, we performed immunogold labelling of 310 WASH. WASH was observed in close proximity to virus particles bound to flat plasma 311 membrane regions (binding), slightly curved membranes associated with virus 312 particles (early pit), and with fully formed HPV16 endocytic pits (late pit) (Figure 4G).

313 In summary, our data supports a model in which WASH acts in a novel function 314 during endocytosis: WASH was recruited to the plasma membrane early during pit 315 formation, stayed associated during all stages of pit maturation (Figure 4G), and 
316 acted at a late stage (Figure 4D) to initiate actin polymerization for vesicle scission

317 during HPV16 endocytosis.

318

\section{The retromer BAR protein SNX2 facilitated HPV16 endocytosis}

320 How is WASH then recruited to virus entry sites? On endosomes, WASH recruitment

321 occurs by interaction of the WASH regulatory complex with the retromer complex

322 (Gomez and Billadeau, 2009; Harbour, Breusegem and Seaman, 2012; Jia et al.,

323 2012). In line with its well established function in endosomal cargo sorting, the

324 retromer has also been implicated in HPV16 retrograde transport to the Golgi

325 (Lipovsky et al., 2013; Popa et al., 2015; Zhang et al., 2018).

327 To analyze whether retromer components would play an additional role in HPV16 328 uptake, we initially used retro-2, a selective inhibitor of retrograde trafficking of e.g. 329 Shiga toxin B from endosomes to the Golgi (Bujny et al., 2007; Popoff et al., 2007;

330 Stechmann et al., 2010). As expected, retro-2 dose-dependently reduced HPV16

331 infection (Figure 5A) (Lipovsky et al., 2013), while herpes simplex virus 1 (HSV-1)

332 infection was unaffected (Nicola et al., 2005) (Figure 5A). HPV16 binding was

333 unchanged in presence of retro-2 (Figure S6A). Hence, decreased infection was not

334 a consequence of perturbed recycling and reduced receptor presentation. Next, we

335 dissected possible effects of retro-2 on HPV16 endocytosis and retrograde

336 trafficking, respectively. First, we selectively targeted trafficking in an inhibitor swap

337 experiment. HPV16 uptake was allowed in presence of $\mathrm{NH}_{4} \mathrm{Cl}$, a weak base that

338 raises the endosomal $\mathrm{pH}$ and prevents onward trafficking of HPV16 (Day, Lowy and

339 Schiller, 2003; Schelhaas et al., 2012). At $12 \mathrm{~h}$ post infection (p.i.)., when a large

340 fraction of virus had reached endosomes, $\mathrm{NH}_{4} \mathrm{Cl}$ was swapped for retro-2 to prevent

341 retrograde transport. HPV16 infection was dose-dependently decreased (Figure 5B), 
342 in line with the requirement for HPV16 endosome-to-Golgi transport (Lipovsky et al.,

343 2013). To assess potential additional effects of retro-2 on HPV16 endocytosis, we

344 analyzed virus uptake in an infectious internalization assay. Cells were infected in

345 presence of retro-2 for $12 \mathrm{~h}$, when extracellular virions were inactivated by a high $\mathrm{pH}$

346 wash and infection by already internalized virions was allowed to continue in absence

347 of retro-2. Uptake of infectious HPV16 was dose-dependently reduced by about $70 \%$

348 in presence of retro-2 (Figure 5C). In conclusion, retro-2 inhibited both retrograde

349 trafficking (Figure 5B) and HPV16 uptake by endocytosis (Figure 5C). To determine

350 the stage of pit formation affected by retro-2 treatment, we performed ultra-thin

351 section TEM. Since retro-2 decreased the number of virus-containing endocytic pits

352 by $90 \%$, it is likely that retro- 2 inhibited the initiation of pit formation (Figure 5D). In

353 conclusion, components of the retrograde trafficking machinery already acted during

354 early steps of HPV16 endocytosis.

356 To specifically address a role for the retromer in either initiation of pit formation or

357 membrane curvature formation, we targeted retromer components by RNAi. The

358 retromer is composed of a cargo-selective complex (CSC) of vacuolar protein sorting

359 (Vps) 26, Vps29, and Vps35, and a dimeric membrane-bending subunit consisting of

360 sorting nexin BAR proteins SNX1/SNX2 and SNX5/SNX6 (Seaman, 2012). As

361 previously observed, RNAi against Vps26, Vps29 and SNX2 strongly reduced

362 infection and suggested that both retromer subunits were required for HPV16

363 infection (Figures 6A and S6B) (Lipovsky et al., 2013; Siddiqa et al., 2018). As

364 control and to differentiate HPV16 endocytosis from macropinocytosis, depletion of

365 CtBP1, a prominent BAR domain containing regulator of macropinocytosis (Liberali et

366 al., 2008; Valente, Luini and Corda, 2013), did not impair HPV16 infection (Figure $3676 \mathrm{~A})$. 
369 Since previous work indicates that depletion of Vps29 is expendable for HPV16

370 uptake but demonstrates an essential role of the CSC in HPV16 endosomal

371 trafficking (Lipovsky et al., 2013, 2015; Popa et al., 2015; Xie et al., 2020), the CSC

372 is unlikely to facilitate HPV16 endocytosis. Recent evidence suggests that retromer

373 BAR proteins can act independent of the CSC on endosomes (Kvainickas et al.,

374 2017; Simonetti et al., 2017; Yong et al., 2020). Hence, we focused our attention on

375 the role of SNX2. While binding of HPV16 to cells was unaffected upon knockdown of

376 SNX2 (Figures S6C and S6D), HPV16 uptake was significantly reduced thereby

377 strengthening a potential role of SNX2 in endocytosis (Figure 6B).

378

379 Similar to WASH, a direct involvement of SNX2 in endocytosis necessitates its 380 presence at endocytic sites. During live cell TIRF-M, SNX2 and HPV16 signals co-

381 localized for more than 50 seconds prior to virus uptake and co-internalized

382 (Figures 6C and 6D, Suppl. Movie 4). Thus, SNX2 resembled WASH dynamics 383 during endocytosis. Immunogold labeling confirmed that SNX2 was indeed present at 384 the same endocytic stages than WASH, namely from virus binding to late stages of 385 pit formation (Figure 6E). Thus, these findings indicated a novel function of the 386 retromer protein SNX2 in facilitating HPV16 endocytosis, likely through membrane 387 bending and/or recruitment of WASH to endocytic sites. 


\section{Discussion}

389 HPV16 uptake occurs by a unique endocytic mechanism, which is distinguished from

390 other mechanisms predominantly in negative terms, i.e., clathrin-, caveolin-,

391 dynamin-, cholesterol-independent and morphologically distinct from

392 macropinocytosis. This work provides evidence for a mechanistic model, in which

393 HPV16 is internalized by inward budding of uncoated endocytic vesicles. Pit

394 formation is facilitated by recruitment of the membrane bending retromer unit and

395 WASH, the latter of which stimulates branched actin polymerization for scission

396 (Figure 7). Hence, our work not only describes an unexpected direct function of

397 SNX2 and WASH in endocytosis, but also delineates that this mechanism follows a

398 stepwise itinerary similar to CME and distinct from macropinocytosis (Figure 7).

399 Since WASH has neither been described at the plasma membrane nor been

400 attributed to any other endocytic pathway, it defines the molecular identity of the

401 unique endocytic mechanism. Thus, we propose to call this pathway WASH-

402 mediated endocytosis (WASH-ME).

403

404 The endocytic landscape includes a variety of pathways, in which endocytic vesicle

405 formation is achieved by different machineries. These pathways typically differ in

406 morphology, key cargos, and molecular features (Doherty and McMahon, 2009).

407 WASH-ME falls in the category of pathways that form endocytic vacuoles by inward

408 budding of the plasma membrane in distinguishable steps. Thus, vesicles are formed

409 by de novo assembly of the endocytic machinery in a modular manner, a mode

410 comparable to CME (Figure 7). Vesicle formation is completed by a scission event.

411 In many endocytic pathways, this is mediated by the large GTPase dynamin. During

412 CME, dynamin polymerizes to form a collar that compresses the vesicle neck by an

413 extensively discussed mechanism leading to membrane fission (Hinshaw and 
414 Schmid, 1995; Takei et al., 1995; Sweitzer and Hinshaw, 1998; Morlot and Roux,

415 2013). In contrast, WASH-ME occurs independently of dynamin (Spoden et al., 2008;

416 Schelhaas et al., 2012) but involves actin polymerization, which coincided with

417 vesicle scission in a timely fashion that resembled dynamin recruitment during CME

418 (Merrifield et al., 2002; Merrifield, 2004). Hence, actin most likely facilitates vesicle

419 scission by a force-driven mechanism rather than as anchoring structure for other

420 scission factors. How actin polymerization creates the force for vesicle scission

421 remains elusive. However, in analogy to observations from CME or endocytosis in

422 Xenopus oocytes, actin polymerization towards the vesicle neck may serve to

423 constrict and propel the vesicle away from the plasma membrane (Bement, Sokac

424 and Mandato, 2003; Sokac et al., 2003; Collins et al., 2011).

426 Endocytic vesicle scission is tightly coupled to membrane remodeling (Dawson, Legg 427 and Machesky, 2006; Shin et al., 2008). This is often coordinated by BAR domain 428 proteins, which sense and stabilize membrane bending by intrinsic curvature (Gallop 429 et al., 2006; Jao et al., 2010; Qualmann, Koch and Kessels, 2011). For instance, 430 SNX9 not only participates in dynamin recruitment (Soulet et al., 2005), but also 431 recruits and directly activates the NPF N-WASP for vesicle scission during CME 432 (Yarar, Waterman-Storer and Schmid, 2007; Shin et al., 2008). Since we 433 demonstrated similar dynamics of SNX2 and WASH at virus entry sites during 434 WASH-ME, perhaps SNX2 aids WASH function in a similar fashion. However, 435 whereas SNX9 and N-WASP are recruited at late stages of CME (Taylor, Perrais and 436 Merrifield, 2011; Kaksonen and Roux, 2018), SNX2 and WASH are already present 437 much prior to vesicle scission. Despite the early recruitment, our findings indicated 438 that WASH promoted actin polymerization only late during vesicle scission. Hence, in 439 contrast to CME, recruitment and activation of the NPF are likely uncoupled in 
440 WASH-ME. How SNX2 recruitment and WASH activation are induced for WASH-ME

441 is unclear. In analogy to their function on endosomes, however, binding of SNX2 to 442 specific phosphoinositides (Zhong et al., 2002; Carlton et al., 2005) and 443 posttranslational modifications of WASH (Hao et al., 2013) are likely involved in their 444 function during WASH-ME. In line with this notion, WASH-ME is controlled by several 445 protein and lipid kinases (Schelhaas et al., 2012; Bannach et al., 2020).

447 How WASH-ME is induced by ligand-receptor interactions is an intriguing question. In addition to mechanistic insights, it would point to the cellular role of WASH-ME and 449 the cargo it internalizes. Likely, WASH-ME is induced only during specific cell states, as WASH is infrequently observed at the plasma membrane. It is reasonable to assume that HPV16 exploits a pathway that is highly active during infection of target cells. These are basal keratinocytes of the mucosal epidermis that become accessible to the virus upon epidermal wounding (Doorbar, 2005; Roberts et al., 454 2007; Aksoy, Gottschalk and Meneses, 2017). Wounding and other cellular responses stimulate epithelial to mesenchymal transition and cell migration, which are accompanied by the remodeling of cell-matrix adhesion complexes, such as focal adhesions and hemidesmosomes (HDs) (Jones, Hopkinson and Goldfinger, 1998; Borradori and Sonnenberg, 1999; Webb, Parsons and Horwitz, 2002; Ezratty, 459 Partridge and Gundersen, 2005; Walko, Castañón and Wiche, 2015). To date, little is 460 known about the dynamics of HD remodeling during cell migration, but HD containing plasma membrane domains can be rapidly endocytosed upon detachment from the 462 underlying extracellular matrix (ECM) (Owaribe et al., 1990). Since HDs contain 463 integrin $\alpha 6$ and CD151, which are part of the HPV16 entry platform (Scheffer et al., 464 2013; Walko, Castañón and Wiche, 2015; Mikuličić et al., 2019), WASH-ME may 465 promote HD uptake during wound healing to aid cell migration and thereby wound 
466 closure. Another essential process during wound healing is matrix remodeling.

467 Interestingly, WASH was previously implicated in apical endocytosis of extracellular

468 material in the Drosophila airway epithelium during embryogenesis (Tsarouhas et al.,

469 2019). Hence, WASH-ME could also contribute to matrix remodeling as a specialized

470 mechanism for internalization of extracellular material.

472 While extensive future research will have to address the cellular role of WASH-ME,

473 its role in pathogen invasion may be of considerable interest as well. For instance,

474 WASH is recruited to the plasma membrane during Salmonella infection presumably

475 serving as one of several entry pathways (Hänisch et al., 2010). Moreover, WASH

476 regulatory complex protein family with sequence similarity 21 co-localizes with a 477 subset of VV particles in plasma membrane lipid rafts (Huang et al., 2008). Thus, 478 WASH-ME may constitute an important entry route for pathogens. Accordingly, 479 viruses such as IAV and LCMV exploit pathways with endocytic vacuole morphology 480 and mechanistic requirements similar yet not identical to HPV16 endocytosis 481 (Sieczkarski and Whittaker, 2002; Quirin et al., 2008; Rojek, Perez and Kunz, 2008; 482 de Vries et al., 2011).

484 The identification of SNX2 and WASH marks the beginning of a deeper 485 understanding of the mechanism underlying WASH-ME. Future research will aim to 486 reveal its cellular function, to identify additional components and the functional 487 interplay of the machinery in this uncharacterized new endocytic pathway to better 488 understand the mechanism of WASH-ME. 


\section{Limitations of the study}

494 This work describes a novel function of the NPF WASH in regulating actin 495 polymerization for vesicle scission, thereby defining a molecular identity of a unique 496 endocytic pathway exploited by HPV16 for infection. Based on the fact that HPV16

497 uptake through WASH-ME occurs highly asynchronously over many hours, only very

498 few endocytic events are observable per cell at any given time post infection. Thus, 499 identification of endocytic structures proves to be challenging. For example, 500 colocalization analysis with marker proteins leads to statistically inconclusive results. 501 While dynamic association of cellular proteins with HPV16 during uptake delineated 502 by TIRF-M as well as identification of endocytic pits by EM together with immunogold 503 labelling of cellular proteins demonstrated WASH and SNX2 localization and

504 recruitment to endocytic pits events, the sample size of these experiments is limited if 505 compared to typical colocalization experiments. 


\section{Acknowledgements}

507 We thank I. Fels (Institute of Cellular Virology, Münster, Germany) for technical

508 support during virus production and U. Westerkamp as well as M. Dominguez (both

509 Institute of Cellular Virology, Münster, Germany) for assistance with experiments. We

510 are indebted to numerous individuals for sharing valuable reagents as indicated in

511 material and methods. We acknowledge the Infectious Diseases Imaging Platform at

512 the University Hospital Heidelberg, K. Richter from the Core Facility Unit Electron

513 Microscopy at the DKFZ Heidelberg and S. Hillmer from the Electron Microscopy

514 Core Facility of the University Heidelberg for their technical support. This work was

515 supported by funding of the German Research Foundation (DFG) to MS (grants

516 SCHE 1552 6-2 and INST211/817A09), and to SB (grant BO 4340/2-1).

\section{Author contributions}

519 Conceived and designed experiments: PB, LK, PSV, CB, TS, MS; Performed 520 experiments: PB, LK, PSV, LG, CB, DB, JK; Analyzed data: PB, LK, PSV, LG, CB,

521 DB, JK, SB, TS, MS; Resources: PD, SB, TS, MS; Writing: PB, LK, MS with input 522 from the other authors.

\section{Declaration of interests}

525 The authors declare no competing interests. 


\section{Figure legends}

527 Figure 1 HPV16 endocytosis as an uncoated, inward budding mechanism

528 (A) HaCaT cells were seeded on HPV16-AF568 bound to ECM, treated with $10 \mu \mathrm{g} / \mathrm{ml}$ 529 cytoD or DMSO and unroofed. Fluorescent virus particles imaged by spinning disk 530 microscopy and depicted in the small insets were correlated with structures identified

531 in TEM images of platinum/carbon replicas of unroofed membranes. Arrowheads

532 indicate endocytic pits. (B) Depicted is the percentage of particles associated with the 533 indicated structure \pm variation between the membranes (DMSO: 134 viruses/

5347 membranes; cytoD: 101 viruses/5 membranes). Of note, about $20-25 \%$ of particles 535 were not associated with any prominent structure. (C) HeLa ATCC cells were 536 infected with HPV16-GFP in presence of cytoD. Infection was scored by flow 537 cytometry and normalized to DMSO treated controls. The mean of three experiments $538 \pm$ SD is shown. (D) HeLa ATCC cells treated with $10 \mu \mathrm{g} / \mathrm{ml}$ CytoD or left untreated 539 (ctrl.) were infected with HPV16-GFP and processed for ultra-thin section TEM at $6 \mathrm{~h}$ 540 p.i.. All scale bars are $100 \mathrm{~nm}$.

542 Figure 2 Actin polymerization coincided with HPV16 uptake

543 (A) HeLa ATCC cells were infected with HPV16-GFP, subjected to immunogold

544 labeling of actin on ultra-thin cryosections, and analyzed by TEM. Asterisks indicate 545 HPV16 in endocytic pits. Scale bars are $100 \mathrm{~nm}$. (B) HeLa ATCC cells were 546 transfected with lifeact-EGFP, infected with HPV16-AF594 and imaged by live cell 547 TIRF-M at $1 \mathrm{~h}$ p.i.. Movies were acquired with $0.5 \mathrm{~Hz}$ frame rate for $5 \mathrm{~min}$. HPV16 548 entry events were identified manually after background subtraction and filtering. The 549 yellow box indicates the virus entry event shown as a kymograph (right) and intensity 550 profile (C). Scale bar is $2 \mu \mathrm{m}$. (C) Plotted are the intensity profiles of HPV16 and 551 lifeact (light red/green) as well as moving averages (intense red/green). Values are 
552 depicted relative to the half time of virus loss $(t=0)$. The time points of the onset of

553 actin polymerization (1) and of virus signal loss (2) from the cell surface, as well as of

554 actin peak intensity (3) and of completion of virus uptake (4) were determined

555 manually. (D) Time points were determined for 21 virus entry events as indicated in

556 (C), averaged and are depicted as box plots.

558 Figure 3 Branched actin polymerization mediated HPV16 endocytosis

559 (A) HeLa ATCC cells were infected with HPV16-GFP or VSV-GFP in presence of the

560 formin inhibitor SMIFH2. Infection was scored by flow cytometry, normalized to

561 solvent treated controls and is depicted as mean \pm SD (HPV16: $n=3$, VSV: $n=4)$.

562 (B) HeLa ATCC cells transfected with Scar-W-GFP or Scar-WA-GFP were infected

563 with HPV16-RFP. Infection of transfected cells was analyzed by microscopy,

564 normalized to Scar-W-GFP control cells and is depicted as the mean of three

565 experiments \pm SD. (C) HeLa Kyoto cells were depleted of Arp3 and infected with

566 HPV16-GFP, VV-GFP or SFV. Infection was scored by automated microscopy or

567 flow cytometry, normalized to cells transfected with a non-targeting control siRNA

568 (ctrl.) and is depicted as mean \pm SD (HPV16/SFV: $n=3$, VV: $n=4)$. (D) Arp3

569 depletion was followed by infection with HPV16-pHrodo and live cell spinning disk

570 microscopy at $6 \mathrm{~h}$ p.i.. Shown are average intensity projections of the HPV16-pHrodo

571 signal with cell outlines (yellow), scale bar is $25 \mu \mathrm{m}$. Virus signal intensities per cell

572 were measured using a CellProfiler pipeline and normalized to ctrl. ( $\mathrm{n}=4$, mean

$573 \pm$ SD). Knock down levels were analyzed by Western blotting against Arp3.

574

575 Figure 4 WASH associated early, but acted late during HPV16 uptake

576 (A) HeLa Kyoto cells were depleted of WASH and infected with HPV16-GFP or VV-

577 GFP. Infection was determined by automated microscopy and flow cytometry and 
578 normalized to cells transfected with a control siRNA (ctrl.) ( $n=4$, mean \pm SD.) (B)

579 After depletion of WASH or Arp3, cells were infected with HPV16-pHrodo and

580 imaged live by spinning disk microscopy at $6 \mathrm{~h}$ p.i.. Shown are average intensity 581 projections of the HPV16-pHrodo signal with cell outlines (yellow), scale bar is 25

$582 \mu \mathrm{m}$. The intensity of virus signal per cell was normalized to ctrl. and is depicted as the 583 mean of three experiments \pm SD. (C) EGFP (ctrl.) or EGFP-WASH were expressed 584 in NIH-3T3 wild type and WASH KO cells. Infection with HPV16-RFP was scored by 585 flow cytometry and normalized to wild type cells expressing EGFP (ctrl.). Values are 586 depicted as the mean \pm SD $(n=3)$. (D) NIH-3T3 wild type and WASH KO infected 587 with HPV16-GFP were subjected to ultra-thin section TEM at $6 \mathrm{~h} \mathrm{p.i..} \mathrm{The} \mathrm{number} \mathrm{of}$ 588 virus filled plasma membrane invaginations was determined for 43 cells per cell line 589 in two independent experiments. Total pit numbers were normalized to wild type cells 590 and are depicted as mean \pm SD. Scale bars are $100 \mathrm{~nm}$. (E) HeLa ATCC cells

591 transfected with EGFP-WASH were infected with HPV16-AF647. Cells were imaged 592 by live cell TIRF-M at $1 \mathrm{~h}$ p.i.. Movies were acquired with $0.5 \mathrm{~Hz}$ frame rate for $5 \mathrm{~min}$. 593 HPV16 entry events were identified manually after background subtraction and 594 filtering. Shown is a kymograph of the virus entry event highlighted by the yellow box, 595 and the corresponding EGFP-WASH signal. Scale bar is $2 \mu \mathrm{m}$. (F) Intensity profiles 596 of HPV16 and WASH (light red/green) as well as moving averages (intense $597 \mathrm{red} / \mathrm{green}$ ) of the virus entry event shown in (E) depicted relative to the half time of 598 virus loss $(\mathrm{t}=0) .(\mathrm{G})$ HeLa ATCC cells were transfected with EGFP-WASH and 599 infected with HPV16. At 6 h p.i., cells were subjected to immunogold labeling of GFP 600 (WASH, $15 \mathrm{~nm}$ gold) and actin (10 nm gold) on ultra-thin cryosections analyzed by 601 TEM. Asterisks indicate HPV16 particles, black and white arrowheads indicate 602 WASH staining and the membrane, respectively. Scale bars are $100 \mathrm{~nm}$. 
604 Figure 5 Components of the retrograde trafficking pathway acted early during 605 HPV16 endocytosis

606 (A) HeLa Kyoto cells were treated with retro-2 and infected with HPV16-GFP or

607 HSV-1-GFP. Infection was scored by flow cytometry and normalized to solvent

608 treated controls $(n=3$, mean \pm SD). (B) HeLa Kyoto cells were infected with HPV16-

609 GFP in presence of $10 \mathrm{mM} \mathrm{NH}_{4} \mathrm{Cl}$. At $12 \mathrm{~h}$ p.i., $\mathrm{NH}_{4} \mathrm{Cl}$ was washed out and infection

610 was continued in presence of $100 \mu \mathrm{M}$ retro-2. Infection was scored by flow cytometry

611 at $48 \mathrm{~h}$ p.i. and normalized to solvent treated controls. The mean of three

612 experiments \pm SD is shown. (C) HeLa Kyoto cells were treated with $100 \mu \mathrm{M}$ retro-2

613 and infected with HPV16-GFP. At $12 \mathrm{~h}$ p.i., extracellular virus was inactivated by a

614 basic $\mathrm{pH}$ wash. Infection was continued in absence of the inhibitor and scored by

615 flow cytometry. Depicted is the fraction of infected cells after the basic wash

616 normalized to DMSO treated cells washed with PBS as the mean of three

617 experiments \pm SD. (D) HeLa Kyoto cells were infected with HPV16-GFP in presence

618 of $100 \mu \mathrm{M}$ retro-2 and subjected to ultra-thin section TEM at $6 \mathrm{~h}$ p.i.. Scale bars are

$619100 \mathrm{~nm}$. The number of virus filled plasma membrane invaginations was determined

620 for 34 and 32 cells in untreated and retro-2 treated cells, respectively, in two

621 independent experiments, normalized to control cells and is depicted as the mean \pm 622 SD.

623

624 Figure 6 The retromer protein SNX2 resembled WASH dynamics

625 (A) HeLa Kyoto cells were depleted of Vps26a, Vps29, SNX2 or CtBP1 and infected 626 with HPV16-GFP. Infection was scored by automated microscopy and normalized to 627 ctrl. ( $n=3$, mean \pm SD). (B) Cells were depleted of Arp3 or SNX2 and infected with 628 HPV16-pHrodo followed by live cell imaging at $6 \mathrm{~h}$ p.i.. Shown are average intensity 629 projections of the HPV16-pHrodo signal acquired by spinning disk microscopy. Cell 
630 outlines in yellow were manually created with brightfield images, scale bar is $25 \mu \mathrm{m}$.

631 The intensity of virus signal per cell was normalized to ctrl. and is shown as mean

$632 \pm$ SD (SNX2: $n=3$, Arp3: $n=2$ ). (C) HeLa ATCC cells were transfected with EGFP-

633 SNX2, infected with HPV16-AF647 and imaged live by TIRF-M at $1 \mathrm{~h}$ p.i.. Movies

634 were acquired with $0.5 \mathrm{~Hz}$ frame rate for $5 \mathrm{~min}$. HPV16 entry events were identified

635 manually after background subtraction and filtering. Shown is a kymograph of the

636 virus entry event highlighted with the yellow box and the corresponding EGFP-SNX2

637 signal. Scale bars is $2 \mu \mathrm{m}$. (D) Intensity profiles of HPV16 and SNX2 (light red/green)

638 as well as moving averages (intense red/green) of the virus entry event shown in (C)

639 depicted relative to the half time of virus loss $(t=0)$. (E) HeLa ATCC cells were 640 transfected with EGFP-SNX2, infected with HPV16 and subjected to immunogold

641 labeling of EGFP-SNX2 on ultra-thin cryosections analyzed by TEM at $6 \mathrm{~h}$ p.i.. Black

642 and white arrowheads indicate SNX2 staining and the membrane, respectively, 643 asterisks mark virus particles. Scale bars are $100 \mathrm{~nm}$.

644

645 Figure 7 Model

646 Schematic model of the mechanistic regulation of endocytic vesicle formation during 647 WASH-ME in comparison to CME and macropinocytosis. Additional regulators 648 involved in the latter mechanisms were omitted for clarity. 


\section{Supplemental figure legends}

650 Figure S1 Kinetics of dynamin recruitment during CME resembled actin 651 dynamics during HPV16 uptake (Related to Figure 2)

652 (A) HeLa ATCC cells were co-transfected with mRFP-clathrin light chain (CLC) and 653 EGFP-dynamin 2 (Dyn2). Cells were imaged by live cell TIRF-M. Movies were 654 acquired with $0.5 \mathrm{~Hz}$ frame rate for $5 \mathrm{~min}$. CME events denoted by CLC signal loss 655 were identified manually after background subtraction and filtering. The yellow box 656 indicates the CME event shown as kymograph. Scale bar is $2 \mu \mathrm{m}$. (B) Plotted are the 657 intensity profiles of CLC and Dyn (light red/green) as well as moving averages 658 (intense red/green). Note that due to its additional role in vesicle maturation, Dyn2 659 was already present early during vesicle formation (Loerke et al., 2009; Taylor, 660 Lampe and Merrifield, 2012). A second wave of recruitment was observed for 661 scission and quantified in (C). (C) The onset of Dyn2 recruitment for scission (1) 662 relative to the half time of CLC loss from the cell surface $(t=0)$ as well as the 663 timepoint of the completion of CLC (3) and Dyn2 (2) signal loss were manually 664 determined from intensity profiles. Values from 10 profiles were plotted.

665

666 Figure S2 Unbranched actin polymerization regulated by formins was not 667 required for HPV16 infection (Related to Figure 3)

668 HeLa Kyoto cells were depleted of individual formins and infected with HPV16-GFP. 669 Infection was scored by automated microscopy and normalized to ctrl., siRNAs that 670 reduced cell numbers by more than $80 \%$ were considered cytotoxic $(\dagger)$ and excluded

671 from the analysis. Depicted is the mean of three experiments \pm SD.

672

673 
674 Figure S3 The NPFs N-WASP, WAVE, WHAMM and JMY were dispensable for

675 HPV16 infection (Related to Figure 4)

676 HeLa Kyoto cells were infected with HPV16-GFP after siRNA mediated depletion of

677 N-WASP (A), WAVE1 (B), WAVE2 (C), JMY (D), WHAMM (E). Infection was scored

678 by automated microscopy, normalized to ctrl. and is depicted as the mean \pm SD $(n=$

679 3). (F) Protein expression levels were determined by Western blotting against the 680 siRNA target proteins.

681

682 Figure S4 The WASH WCA domain was essential for HPV16 infection (Related to 683 Figure 4)

684 (A) EGFP (ctrl.), YFP-WASH or YFP-WASH lacking the WCA domain (dWCA) were 685 expressed in NIH-3T3 wild type and WASH KO cells. Cells were infected with 686 HPV16-RFP. Infection was scored by flow cytometry and normalized to wild type 687 cells transfected with EGFP(ctrl.). Values are depicted as mean \pm SD of three 688 independent experiments. (B) U2OS wild type and WASH KO cells were infected 689 with HPV16-GFP. Infection was analyzed by flow cytometry and normalized to wild 690 type cells (mean $\pm S D, n=8$ ). (C) WASH KO in NIH-3T3 and U2OS cells was 691 confirmed by immunoblotting against WASH. (D) HPV16-AF488 was bound to NIH692 3T3 WASH KO cells. Virus pre-incubated with $1 \mathrm{mg} / \mathrm{ml}$ heparin was used as a non693 binding control. At 2 h p.i., virus binding was measured by flow cytometry. Values are 694 shown as relatives to wild type cells and depicted as mean of three experiments $695 \pm$ SD. (E) Binding of HPV16-AF568 was performed as in (D). After fixation, the actin 696 cytoskeleton was stained with phalloidin-Atto488. Shown are maximum intensity 697 projections of z-stacks acquired by spinning disk microscopy, scale bars are $10 \mu \mathrm{m}$. 698 
699 Figure S5 PLA indicated WASH recruitment to virus entry sites (Related to

$700 \quad$ Figure 4)

701 HPV16-AF488 was bound to ECM. HaCaT cells transfected with the entry platform

702 protein HA-CD151 were seeded on top. Close proximity between WASH and HA-

703 CD151 was detected using a PLA and plasma membrane localization was analyzed

704 by TIRF-M. Scale bars are $2 \mu \mathrm{m}$ and $0.5 \mu \mathrm{m}$.

705

706 Figure S6 Retro-2 treatment and SNX2 knock down did not affect HPV16

707 binding (Related to Figures 5 and 6)

708 (A) Confluent HeLa Kyoto cells were infected with HPV16-AF488 or -AF594 for 2 h in

709 presence of $100 \mu \mathrm{M}$ retro-2. Pre-incubation of the virus with $1 \mathrm{mg} / \mathrm{ml}$ heparin was

710 used as a non-binding control. The intensities of fluorescent HPV16 were determined

711 by spinning disk microscopy and normalized to solvent treated control cells. Depicted

712 are mean values from three experiments \pm SD. (B) Depletion of siRNA target proteins

713 was confirmed by Western blotting. (C) HPV16-AF488 was bound to HeLa Kyoto

714 cells depleted of SNX2 for $2 \mathrm{~h}$. Virus binding was determined by flow cytometry.

715 Values are shown as relatives to crtl. (mean of three independent experiments \pm SD).

716 (D) HPV16-AF568 was bound to HeLa Kyoto cells depleted of SNX2 as above.

717 Spinning disk microscopy was employed after actin staining with phalloidin-Atto488.

718 Shown are maximum intensity projections of z-stacks, scale bar is $10 \mu \mathrm{m}$. 


\section{STAR methods}

\section{KEY RESOURCES TABLE}

\begin{tabular}{|c|c|c|}
\hline REAGENT or RESOURCE & SOURCE & IDENTIFIER \\
\hline \multicolumn{3}{|l|}{ Antibodies } \\
\hline mouse anti actin (4F7) & BRICS & bsbs300470 \\
\hline rabbit polyclonal anti Arp3 & (Steffen et al., 2006) & $\mathrm{N} / \mathrm{A}$ \\
\hline mouse monoclonal (11G5a) anti CD151 & Bio Rad & MCA1856GA \\
\hline mouse monoclonal (3) anti CtBP1 & Becton Dickinson & 612042 \\
\hline rabbit polyclonal anti ERK2 (C-14) & Santa Cruz Biotechnology & sc-154 \\
\hline rabbit polyclonal anti GFP & Abcam & ab290 \\
\hline mouse monoclonal anti HA (16B12) & Covance & MMS-101R \\
\hline goat polyclonal anti JMY (L-16) & Santa Cruz Biotechnology & sc-10027 \\
\hline primary rabbit antiserum "Lady Di" against SFV E1/2 & $\begin{array}{l}\text { kind gift from A. Helenius } \\
\text { (ETH Zurich, Switzerland), } \\
\text { (Singh and Helenius, 1992) }\end{array}$ & $\mathrm{N} / \mathrm{A}$ \\
\hline mouse monoclonal (13) anti SNX2 & Becton Dickinson & 611308 \\
\hline mouse monoclonal (B-5-1-2) anti tubulin & Sigma-Aldrich & T5168 \\
\hline rabbit polyclonal anti Vps26a & Abcam & Ab137447 \\
\hline rabbit polyclonal anti Vps29 & Abcam & Ab98929 \\
\hline rabbit polyclonal anti WASH & $\begin{array}{l}\text { kind gift from A. Gautreau } \\
\text { (Institut Polytechnique De } \\
\text { Paris, Paris, France), } \\
\text { (Derivery et al., 2009) }\end{array}$ & $\mathrm{N} / \mathrm{A}$ \\
\hline rabbit polyclonal anti WASH & Atlas Antibodies & HPA002689 \\
\hline rabbit polyclonal anti WAVE1 & Sigma-Aldrich & W2142 \\
\hline rabbit polyclonal anti WAVE2 $(\mathrm{H}-110)$ & Santa Cruz Biotechnology & sc-33548 \\
\hline rabbit polyclonal anti WHAMM (K-13) & Santa Cruz Biotechnology & sc-136951 \\
\hline goat anti-mouse AF488 lgG & Thermo Fisher Scientific & A-11029 \\
\hline goat anti-mouse AF647 lgG & Thermo Fisher Scientific & A-21235 \\
\hline goat anti-rabbit AF488 $\lg G$ & Thermo Fisher Scientific & A-11034 \\
\hline rabbit anti mouse $\lg G+\lg M$ & Dianova & $315-005-048$ \\
\hline sheep anti-mouse HRP linked IgG & GE Healthcare & NA931 \\
\hline donkey anti-rabbit HRP linked IgG & GE Healthcare & NA934 \\
\hline Duolink In Situ PLA Probe anti-mouse PLUS & Sigma-Aldrich & DUO92001 \\
\hline Duolink In Situ PLA Probe anti-rabbit MINUS & Sigma-Aldrich & DUO92005 \\
\hline Protein A gold $10 \mathrm{~nm}$ & CMC, UMC Utrecht & $\mathrm{N} / \mathrm{A}$ \\
\hline Protein A gold $15 \mathrm{~nm}$ & CMC, UMC Utrecht & $\mathrm{N} / \mathrm{A}$ \\
\hline \multicolumn{3}{|l|}{ Bacterial and Virus Strains } \\
\hline E. coli DH5a & $\begin{array}{l}\text { kind gift from A. Helenius } \\
\text { (ETH Zurich, Switzerland) }\end{array}$ & $\mathrm{N} / \mathrm{A}$ \\
\hline HPV16-GFP pseudoviruses (PsVs) & $\begin{array}{l}\text { (Buck and Thompson, } \\
\text { 2007) }\end{array}$ & $\mathrm{N} / \mathrm{A}$ \\
\hline HPV16-RFP PsVs & (Johnson et al., 2009) & $\mathrm{N} / \mathrm{A}$ \\
\hline HSV-1-EGFP $\left(17\right.$ syn $\left.^{+}\right)$ & $\begin{array}{l}\text { kind gift from W. Hafezi } \\
\text { (University of Münster, } \\
\text { Germany) (Hafezi et al., } \\
\text { 2012) }\end{array}$ & $\mathrm{N} / \mathrm{A}$ \\
\hline SFV (prototype strain) & $\begin{array}{l}\text { kind gift from A. Helenius } \\
\text { (ETH Zurich, Switzerland), } \\
\text { (Marsh and Helenius, 1980) }\end{array}$ & $\mathrm{N} / \mathrm{A}$ \\
\hline VSV-GFP (Indiana) & $\begin{array}{l}\text { kind gift from A. Helenius } \\
\text { (ETH Zurich, Switzerland) } \\
\text { (Johannsdottir et al., 2009) }\end{array}$ & $\mathrm{N} / \mathrm{A}$ \\
\hline
\end{tabular}




\begin{tabular}{|c|c|c|}
\hline VV-GFP (Western Reserve) & $\begin{array}{l}\text { kind gift from J. Mercer } \\
\text { (UCL London, UK), (Mercer } \\
\text { and Helenius, 2008) }\end{array}$ & $\mathrm{N} / \mathrm{A}$ \\
\hline \multicolumn{3}{|c|}{ Chemicals, Peptides, and Recombinant Proteins } \\
\hline AGAR 100 Resin kit (Epoxy resin) & Agar Scientific & R1031 \\
\hline Alexa Fluor 488 succinimidyl ester & Thermo Fisher Scientific & A20000 \\
\hline Alexa Fluor 568 succinimidyl ester & Thermo Fisher Scientific & A20003 \\
\hline Alexa Fluor 594 succinimidyl ester & Thermo Fisher Scientific & A20004 \\
\hline $\begin{array}{l}\text { Alexa Fluor } 647 \text { succinimidyl ester } \\
\text { bafilomycin } \mathrm{A} 1\end{array}$ & $\begin{array}{l}\text { Thermo Fisher Scientific } \\
\text { Applichem }\end{array}$ & $\begin{array}{l}\text { A20006 } \\
\text { A7823 }\end{array}$ \\
\hline Brij-58 & Sigma-Aldrich & P5884 \\
\hline cytochalasin D & Sigma-Aldrich & C8273 \\
\hline glutaraldehyde, EM grade & Polysciences & 01909 \\
\hline heparin & Sigma-Aldrich & H4784 \\
\hline Lipofectamine 2000 & Invitrogen & 11668019 \\
\hline Lipofectamine RNAi max & Invitrogen & 13778075 \\
\hline osmium tetroxide & $\begin{array}{l}\text { Electron Microscopy } \\
\text { Sciences }\end{array}$ & 19100 \\
\hline paraformaldehyde, EM grade & Polysciences & 00380 \\
\hline phalloidin-Atto488 & Sigma-Aldrich & 49409 \\
\hline pHrodo Red succinimidyl ester & Thermo Fisher Scientific & P36600 \\
\hline retro-2 & ChemBridge & 5374762 \\
\hline RedDot2 & VWR & $40061-1$ \\
\hline ROCK inhibitor & Becton Dickinson & Y-27632 \\
\hline SMIFH2 & Sigma-Aldrich & S4826 \\
\hline uranyl acetate & Polysciences & 21447 \\
\hline X-tremeGENE ${ }^{1 \mathrm{In}} 9$ & Merck & XTG9-RO \\
\hline \multicolumn{3}{|l|}{ Critical Commercial Assays } \\
\hline Duolink In Situ Detection Reagents Red & Sigma-Aldrich & DU092008 \\
\hline \multicolumn{3}{|l|}{ Experimental Models: Cell Lines } \\
\hline Hamster: BHK-21-Helsinki & $\begin{array}{l}\text { kind gift from A. Helenius } \\
\text { (ETH Zurich, Switzerland) } \\
\text { (Johannsdottir et al., 2009) }\end{array}$ & $\bar{N} / \mathrm{A}$ \\
\hline Hamster: BHK-21 & $\begin{array}{l}\text { kind gift from W. Hafezi } \\
\text { (University of Münster, } \\
\text { Germany) (Hafezi et al., } \\
\text { 2012) }\end{array}$ & $\bar{N} / \mathrm{A}$ \\
\hline African green monkey: BSC-40 & $\begin{array}{l}\text { kind gift from J. Mercer } \\
\text { (UCL London, UK), } \\
\text { (Brockman and Nathans, } \\
\text { 1974) }\end{array}$ & $\mathrm{N} / \mathrm{A}$ \\
\hline Human: HaCaT & (Boukamp et al., 1988) & $\mathrm{N} / \mathrm{A}$ \\
\hline Human: HeLa ATCC & ATCC (Manassas, USA) & $\mathrm{N} / \mathrm{A}$ \\
\hline Human: HeLa Kyoto & $\begin{array}{l}\text { kind gift from L. Pelkmans } \\
\text { (ETH Zurich, Switzerland), } \\
\text { (Landry et al., 2013) }\end{array}$ & $\mathrm{N} / \mathrm{A}$ \\
\hline Mouse: NIH 3T3 & ATCC (Manassas, USA) & $\mathrm{N} / \mathrm{A}$ \\
\hline $\begin{array}{l}\text { Mouse: NIH 3T3 WASH KOs } \\
\text { Human: U2OS }\end{array}$ & $\begin{array}{l}\text { this study } \\
\text { German collection of } \\
\text { Microorganisms and Cell } \\
\text { Cultures (DSMZ, } \\
\text { Braunschweig, Germany) }\end{array}$ & $\begin{array}{l}\text { N/A } \\
\text { N/A }\end{array}$ \\
\hline Human: U2OS WASH KOs & this study & $\mathrm{N} / \mathrm{A}$ \\
\hline Non-human, primate: Vero & ATCC (Manassas, USA) & $\mathrm{N} / \mathrm{A}$ \\
\hline
\end{tabular}




\begin{tabular}{|c|c|c|}
\hline Human: 293 TT & $\begin{array}{l}\text { kind gift from J. Schiller } \\
\text { (NIH, Bethesda, USA) } \\
\text { (Buck et al., 2005) }\end{array}$ & $\mathrm{N} / \mathrm{A}$ \\
\hline \multicolumn{3}{|l|}{ Oligonucleotides } \\
\hline \multicolumn{3}{|l|}{ refer to supplemental table 1 for siRNAs } \\
\hline $\begin{array}{l}\text { single guide RNA (sgRNA) WASH exon } 2 \text { (murine, } \\
\text { top strand) }\end{array}$ & $\begin{array}{l}\text { GCGACGAGAGGAGGCAA } \\
\text { TCC }\end{array}$ & \\
\hline sgRNA WASH exon 2 (murine, bottom strand) & $\begin{array}{l}\text { GGATTGCCTCCTCTCGTC } \\
\text { GC }\end{array}$ & \\
\hline sgRNA WASH exon 4 (human, top strand) & $\begin{array}{l}\text { TCTTCACGGGCGCCCAG } \\
\text { GAC }\end{array}$ & \\
\hline sgRNA WASH exon 4 (human, bottom strand) & $\begin{array}{l}\text { GTCCTGGGCGCCCGTGA } \\
\text { AGA }\end{array}$ & \\
\hline sgRNA WASH exon 5 (human, top strand) & $\begin{array}{l}\text { GTGTGCGTGAGCACCAA } \\
\text { GCC }\end{array}$ & \\
\hline sgRNA WASH exon 4 (human, bottom strand) & $\begin{array}{l}\text { GGCTTGGTGCTCACGCA } \\
\text { CAC }\end{array}$ & \\
\hline murine WASH sequencing primer (forward) & $\begin{array}{l}\text { ATAGGCAGAGGGGTGAG } \\
\text { TGT }\end{array}$ & \\
\hline murine WASH sequencing primer (reverse) & $\begin{array}{l}\text { ACACTGGGCATTAGTTG } \\
\text { GGT }\end{array}$ & \\
\hline M13r primer for TOPO vector & CAGGAAACAGCTATGAC & \\
\hline \multicolumn{3}{|l|}{ Recombinant DNA } \\
\hline Plasmid: pSpCas9(BB)-2A-GFP (pX458) & Addgene & 48138 \\
\hline Plasmid: pEGFP C1_CD151 & $\begin{array}{l}\text { kind gift from L. Florin } \\
\text { (Johannes Gutenberg } \\
\text { University Mainz, Germany) } \\
\text { (Liu et al., 2007) }\end{array}$ & $\mathrm{N} / \mathrm{A}$ \\
\hline Plasmid: pcDNA3 HA_CD151 & this study & $\mathrm{N} / \mathrm{A}$ \\
\hline Plasmid: pmRFP C3_Clc & $\begin{array}{l}\text { kind gift from A. Helenius } \\
\text { (ETH Zurich, Switzerland) }\end{array}$ & $\mathrm{N} / \mathrm{A}$ \\
\hline Plasmid: pEGFP-Dyn2 & $\begin{array}{l}\text { kind gift from M. A. McNiven } \\
\text { (Mayo Clinic, Rochester, } \\
\text { USA) } \\
\text { (Cao, Garcia and Mcniven, } \\
\text { 1998) }\end{array}$ & $\mathrm{N} / \mathrm{A}$ \\
\hline Plasmid: pClneoEGFP & $\begin{array}{l}\text { kind gift from C. Buck (NIH, } \\
\text { Bethesda, USA) (Buck and } \\
\text { Thompson, 2007) }\end{array}$ & $\mathrm{N} / \mathrm{A}$ \\
\hline Plasmid: Lifeact_EGFP-N1 & $\begin{array}{l}\text { kind gift from R. Wedlich- } \\
\text { Söldner (University of } \\
\text { Münster, Germany) (Riedl } \\
\text { et al., 2008) }\end{array}$ & $\mathrm{N} / \mathrm{A}$ \\
\hline Plasmid: pEGFP C1 & $\begin{array}{l}\text { kind gift from A. Helenius } \\
\text { (ETH Zurich, Switzerland) }\end{array}$ & $\mathrm{N} / \mathrm{A}$ \\
\hline Plasmid: $p R w B$ & $\begin{array}{l}\text { kind gift from C. Buck (NIH, } \\
\text { Bethesda, USA) (Johnson } \\
\text { et al., 2009) }\end{array}$ & $\mathrm{N} / \mathrm{A}$ \\
\hline Plasmid: pEGFP C1_Scar-W-GFP & (Machesky and Insall, 1998) & $\mathrm{N} / \mathrm{A}$ \\
\hline Plasmid: pEGFP-C1_Scar-WA-GFP & (Machesky and Insall, 1998) & $\mathrm{N} / \mathrm{A}$ \\
\hline Plasmid: pcDNA5_RT-PC-EGFP-SNX2 & $\begin{array}{l}\text { kind gift from A. Gautreau } \\
\text { (Institut Polytechnique De } \\
\text { Paris, France), (Helfer et } \\
\text { al., 2013) }\end{array}$ & N/A \\
\hline Plasmid: pcDNA5_EGFP-WASH & (Derivery et al., 2009) & $\mathrm{N} / \mathrm{A}$ \\
\hline Plasmid: $\mathrm{pcCM} 3 \mathrm{H} 1 \mathrm{p}$ YFP WASH wild type & $\begin{array}{l}\text { kind gift from D. Billadeau } \\
\text { (Mayo Clinic, Rochester, } \\
\text { USA)), (Gomez and } \\
\text { Billadeau, 2009) }\end{array}$ & $\mathrm{N} / \mathrm{A}$ \\
\hline
\end{tabular}




\begin{tabular}{|c|c|c|}
\hline Plasmid: pCMS3H1p YFP WASH dWCA & $\begin{array}{l}\text { kind gift from D. Billadeau } \\
\text { (Mayo Clinic, Rochester, } \\
\text { USA)), (Gomez and } \\
\text { Billadeau, 2009) }\end{array}$ & $\mathrm{N} / \mathrm{A}$ \\
\hline Plasmid: p16SheLL & $\begin{array}{l}\text { kind gift from C. Buck (NIH, } \\
\text { Bethesda, USA) (Buck et } \\
\text { al., 2005) }\end{array}$ & $\mathrm{N} / \mathrm{A}$ \\
\hline \multicolumn{3}{|l|}{ Software and Algorithms } \\
\hline Adobe Illustrator & Adobe Inc. & version 16.0.4 \\
\hline Adobe Photoshop & $\begin{array}{l}\text { Adobe } \\
\text { Inc. }\end{array}$ & $\begin{array}{l}\text { C4, extended } \\
\text { version } 11.0\end{array}$ \\
\hline Affinity Designer & Serif (Europe) Ltd & version 1.7.3 \\
\hline ApE - A plasmid Editor & $\begin{array}{l}\text { M. Wayne Davis } \\
\text { (https://jorgensen.biology.ut } \\
\text { ah.edu/wayned/ape/) }\end{array}$ & version 1.11 \\
\hline BD CellQuest Pro & Becton Dickinson & version 5.2 \\
\hline CellProfiler & (Kamentsky et al., 2011) & version 2.1 .1 \\
\hline CellSens Dimension & Olympus & version 2.3 \\
\hline FACSDiva & Becton Dickinson & version 6.1 \\
\hline Fiji (ImageJ) & (Schindelin et al., 2012) & $\begin{array}{l}\text { versions } \\
2.0 .0 / 1.50 \mathrm{~g} \text { and } \\
2.1 .0 / 1.53 \mathrm{c}\end{array}$ \\
\hline FlowJo & Becton Dickinson & $\begin{array}{l}\text { versions } 8.8 .6 \text { and } \\
10.6 .1\end{array}$ \\
\hline guavaSoft & Merck & version 3.1.1 \\
\hline iTEMFEl & FEl/Olympus & version 5.2 \\
\hline $\begin{array}{l}\text { MATLAB } \\
\text { MATLAB InfectionCounter Program }\end{array}$ & $\begin{array}{l}\text { MathWorks } \\
\text { (Snijder et al., 2012) }\end{array}$ & $\begin{array}{l}\text { version R2015a, } \\
8.5 .0 .197613 \\
\text { version blue, } \\
\text { release B3 }\end{array}$ \\
\hline Metamorph (Spinning disk) & Molecular Devices & version 7.7 .2 \\
\hline Metamorph (IX71 TIRF) & Molecular Devices & version 7.7 .1 \\
\hline Microsoft Excel & Microsoft Corporation & $\begin{array}{l}\text { versions } 15.41 .0 \\
\text { and } 16.40\end{array}$ \\
\hline Prism & GraphPad Software, Inc. & version $6.0 f$ \\
\hline SnapGene Viewer & SnapGene & version 2.6 .2 \\
\hline Tecnai software & $\begin{array}{l}\text { FEl/Thermo Fisher } \\
\text { Scientific }\end{array}$ & version 3.1 .3 \\
\hline Volocity software & PerkinElmer & version 6.3 \\
\hline
\end{tabular}

\section{RESOURCE AVAILABILITY}

\section{Lead Contact}

724 Further information and requests for resources should be directed and will be fulfilled

725 by the lead contact, Mario Schelhaas (schelhaas@uni-muenster.de). 


\section{Materials Availability}

731 Plasmids generated in this study are available from the lead contact. WASH knock

732 out cell lines described in this study are available from Theresia Stradal

733 (Theresia.Stradal@helmholtz-hzi.de) with a completed Material Transfer Agreement.

\section{Data and Code Availability}

736 All data reported in this paper will be shared by the lead contact upon request. The

737 paper does not report original code. Any additional information required to reanalyze

738 the data reported in this paper is available from the lead contact upon request.

739

\section{EXPERIMENTAL MODEL AND SUBJECT DETAILS}

\section{$741 \quad$ Cell lines}

742 HeLa ATCC, HeLa Kyoto (both female origin), HaCaT cells (male origin) and non-

743 human, primate Vero cells (female origin) were cultured in high glucose Dulbecco's

744 modified eagle medium (DMEM, D5796 Sigma-Aldrich) supplemented with 10\% fetal

745 bovine serum (FBS). 293TT cells (female origin) were grown in DMEM with 10\% FBS

746 and $400 \mu \mathrm{g} / \mu \mathrm{l}$ hygromycin B. U2OS wild type and WASH KO cells (female origin) as

747 well as murine NIH 3T3 wild type and WASH KO cells (male origin) were cultured in

748 DMEM supplemented with 10\% FBS and 1\% non-essential amino acids (NEAA).

749 Primate, non-human BSC-40 cells (sex unspecified) were grown in DMEM

750 supplemented with 10\% FBS, 5\% NEAA and 5\% sodium pyruvate. Hamster BHK-21

751 cells (male origin) were cultured in Glasgow's minimum essential medium (GMEM)

752 supplemented with $10 \%$ FBS. All cells were cultivated in a humidified atmosphere at $753 \quad 37^{\circ} \mathrm{C}$ and $5 \% \mathrm{CO}_{2}$ and routinely tested for mycoplasma contamination. 


\section{Bacteria strains}

757 Chemocompetent E. coli $\mathrm{DH} 5$ a used for plasmid preparation were grown in lysogeny

758 broth (LB) medium supplemented with antibiotics at $37^{\circ} \mathrm{C}$ and $210 \mathrm{rpm}$.

759

\section{$760 \quad$ Virus strains}

761 VV-GFP (Western Reserve) containing a fluorescent version of the core protein A5

762 was propagated and titrated on BSC-40 cells in standard growth medium and purified

763 as described previously (Mercer and Helenius, 2008). HSV-1-EGFP (17syn+)

764 expressing EGFP under control of the cytomegalovirus promoter was propagated on

765 BHK-21 cells and titrated on Vero cells, both grown in GMEM supplemented with $2 \%$

766 FBS, as described previously (Schelhaas et al., 2003; Hafezi et al., 2012). SFV

767 (prototype strain) was propagated and titrated on BHK-21-Helsinki cells as previously

768 described (Marsh, Kielian and Helenius, 1984). Infection was carried out in GMEM

769 supplemented with 10\% FBS and 10\% Tryptose Broth. VSV-GFP (Indiana)

770 expressing an additional transgene encoding GFP was propagated on BHK-21-

771 Helsinki cells grown in GMEM supplemented with $10 \mathrm{mM}$ HEPES (pH 6.5). At $1 \mathrm{~h}$

772 p.i., GMEM supplemented with $30 \mathrm{mM}$ HEPES (pH 7.3) and 10\% Tryptose

773 Phosphate Broth and 1\% FBS was added, as previously described (Johannsdottir et

774 al., 2009). The virus was titrated on BHK-21-Helsinki cells grown in RPMI

775 supplemented with $30 \mathrm{mM}$ HEPES (pH 6.5). HPV16 PsVs were produced as

776 described in the method details.

777

778 METHOD DETAILS

779 Cloning and plasmid purification

780 Cloning of HA-CD151 construct 
781 Lentiviral expression constructs of HA-tagged CD151 were a kind gift from M. Hemler

782 (Dana Farber Cancer Institute and Harvard Medical School, Boston, USA) (Hwang,

783 Takimoto and Hemler, 2019). For subcloning, HA-CD151 was cut from the lentiviral

784 vector using EcoRl and $X b a l$ for $2 \mathrm{~h}$ at $37^{\circ} \mathrm{C}$. A pcDNA3 expression vector was cut

785 using the same enzymes and conditions. For purification, both samples were run on

786 a $2 \%$ acrylamide gel. The DNA was visualized with ethidium bromide and bands

787 representing the cut pcDNA3 backbone as well as HA-CD151 (insert) were isolated

788 from the gel. Gel extraction was performed with a NucleoSpin Gel and PCR Clean-up

789 kit (Macherey-Nagel). DNA concentrations were determined with help of a $1.5 \%$

790 agarose gel by comparison to the marker bands (Gene Ruler $1 \mathrm{~kb}$ DNA ladder,

791 Thermo Scientific). Insert and backbone were ligated by incubation with the T4 ligase

792 overnight at $16^{\circ} \mathrm{C}$.

793

\section{Plasmid purification}

795 Chemocompetent E. coli DH5a were incubated with $5 \mu$ ligation product for $10 \mathrm{~min}$

796 on ice. Heat shock was performed by $60-90 \mathrm{sec}$ incubation at $42^{\circ} \mathrm{C}$. Immediately

797 afterwards, bacteria were incubated on ice for 5 min before LB medium was added.

798 Bacteria were grown at $37^{\circ} \mathrm{C}$ and $350 \mathrm{rpm}$ for $30-60 \mathrm{~min}$ and plated on LB agar

799 plates with antibiotics using inoculation loops. Plates were incubated overnight at

$80037^{\circ} \mathrm{C}$ and inspected for colony growth the next day. For HA-CD151 cloning, several

801 overnight cultures with LB supplemented with antibiotics were inoculated with one

802 colony each. Plasmids were purified using the NucleoSpin Plasmid kit (Macherey-

803 Nagel) and sent for sequencing by Eurofins Genomics (Luxembourg). Sequence

804 analysis was performed with ApE. For use in experiments, plasmids were purified

805 using the NucleoBond Xtra Maxi kit (Macherey-Nagel). 
sgRNA design and cloning

sgRNAs were provided by a CRISPR design tool (CRIPR.mit.edu or CCTop).

811 Specifically, the murine gene was disrupted by sgRNAs targeting exon 2 and the

812 human gene by simultaneously targeting exons 4 and 5 (key resources table). Top

813 and bottom strands of sgRNAs were annealed using T4 ligase for $30 \mathrm{~min}$ at $37^{\circ} \mathrm{C}$ and

814 cloned into pSpCas9(BB)-2A-GFP (pX458) by digestion with Bbsl and ligation with

815 T4 ligase $\left(6\right.$ cycles: $37^{\circ} \mathrm{C}$ for $5 \mathrm{~min}, 21^{\circ} \mathrm{C}$ for $5 \mathrm{~min}$ ). Residual linearized DNA was

816 removed by treatment with PlasmidSafe exonuclease at $37^{\circ} \mathrm{C}$ for $30 \mathrm{~min}$. Chemically

817 competent E.coli $\mathrm{DH} 5 \mathrm{a}$ were transformed with the ligation product as described 818 above.

820 Generation and characterization of WASH KO cell lines with CRISPR/Cas9

$821 \mathrm{NIH}-3 \mathrm{~T} 3$ and U2OS cells were plated in 6-well plates and maintained in DMEM 822 (4.5 g/L glucose, Invitrogen, Germany) supplemented with 10\% (v/v) FBS (Sigma, 823 Germany), $1 \mathrm{mM}$ sodium pyruvate, $1 \mathrm{x}$ non-essential amino acids and $2 \mathrm{mM} \mathrm{L-}$ 824 glutamine at $37^{\circ} \mathrm{C}$ in a humidified $7.5 \%$ CO2-atmosphere overnight. Cells were 825 genome edited using the CRISPR/Cas9 technology (Ran et al., 2013) to generate 826 WASH KO cell lines. Selected sgRNAs (key resources table) were cloned into pX458 827 allowing simultaneous expression of sgRNA, Cas9 and selection via EGFP 828 expression as described above. The resulting constructs were transfected into $\mathrm{NIH}$ 8293 T3 or U2OS cells, respectively. Plasmids $(1 \mu \mathrm{g})$ and X-tremeGene ${ }^{\mathrm{TM}} 9(3 \mu \mathrm{l})$ were 830 diluted in $100 \mu \mathrm{l}$ optiMEM, incubated for $30 \mathrm{~min}$ at room temperature and added to 831 cells for $16-24 \mathrm{~h}$. Transfection efficiency was monitored using an EVOS® FL Cell 832 Imaging System (Thermo Fisher, Germany). Cells were grown to confluence and 
833 subsequently single, GFP-positive cells were sorted into 96-well plates by flow

834 cytometry using a FACSAria II instrument (BD Biosciences) and FACSDiva software.

835 Sorted cells were maintained in growth medium supplemented with penicillin (50

836 Units $/ \mathrm{ml}) /$ streptomycin $(50 \mathrm{mg} / \mathrm{ml}$ ) (Thermo Fisher Scientific) and containing $30 \%$

837 conditioned medium and $10 \mu \mathrm{M}$ ROCK inhibitor (BD Biosciences). After

838 approximately 10 days, clones were picked from single wells and expanded. Derived

839 cell clones were screened for the absence of WASH expression by Western blotting

840 and NIH-3T3 clones lacking detectable amounts of WASH were subjected to

841 genomic sequencing as described (Kage et al., 2017). Cells from confluent $6 \mathrm{~cm}$

842 dishes were trypsinized, pelleted and lysed by overnight incubation in lysis buffer

843 (100 mM Tris pH 8.5, 5 mM EDTA, 0.2\% SDS, $200 \mathrm{mM} \mathrm{NaCl,} 20 \mathrm{mg} / \mathrm{ml}$ proteinase

$844 \mathrm{~K})$ at $55^{\circ} \mathrm{C}$. Nucleic acid extraction was performed by ethanol precipitation. Addition

845 of $700 \mu \mathrm{l} 100 \%$ ice cold ethanol was followed by centrifugation at $16,000 \times \mathrm{g}$ at $4^{\circ} \mathrm{C}$

846 for $30 \mathrm{~min}$. The pellet was washed with $400 \mu \mathrm{l} 70 \%$ ice-cold ethanol and samples

847 were dried at $45^{\circ} \mathrm{C}$ for $20 \mathrm{~min}$. DNA was dissolved in $100 \mu$ l deionized water at $4^{\circ} \mathrm{C}$

848 overnight and served as template in PCR using GoTaq G2 flexi DNA polymerase.

849 The selected primer pair (key resources table) revealed a PCR product of 330 base

850 pairs. PCR products were examined on $2 \%$ agarose gels and appropriate samples

851 purified with a NucleoSpin Gel and PCR clean-up kit (Macherey-Nagel). DNA

852 fragments were cloned into a zero blunt TOPO vector (Zero Blunt TOPO Cloning Kit

853 for Sequencing, Invitrogen) for $5 \mathrm{~min}$ at room temperature. After transformation as

854 above, single bacterial colonies were inoculated overnight and plasmid DNA purified

855 using NucleoSpin Plasmid kit (Macherey-Nagel). Sequencing of isolated plasmid

856 DNA was carried out by MWG-Biotech using a standard M13r sequencing primer

857 (key resources table). Clones were examined for frameshift mutations and mono- or

858 biallelic deletions/insertions using SnapGene Viewer software. Mutations or deletions 
859 generating stop codons shortly downstream of the target site were defined as 'null'

860 alleles. Cell populations exclusively harboring such alleles out of more than 50

861 sequencing reactions were selected for further analyses. For WASH targeted clones

862 of murine NIH-3T3 cells, three clones were identified that did not display a wild type

863 allele in more than ten sequencing reactions. All clones showed similar effects on

864 HPV16 infection, thus only one clone is shown in this study. For WASH targeted

865 clones of human U2OS cells no clear sequencing result was obtained probably due

866 to pseudogenes being targeted by sequencing primers.

867

868 Western blotting

869 For analysis of protein amounts after siRNA treatment or CRISPR/Cas9 mediated

$870 \mathrm{KO}$, lysates were prepared in 2x sample buffer (4\% SDS, $20 \%$ glycerol, $0.01 \%$

871 bromophenol blue, $100 \mathrm{mM}$ Tris $\mathrm{HCl}(\mathrm{pH}$ 6.8), $200 \mathrm{mM}$ DTT). Samples were

872 denatured for $5 \mathrm{~min}$ at $95^{\circ} \mathrm{C}$ and loaded on polyacrylamide gels. For stacking gels,

$8735 \%$ polyacrylamide was used and separating cells were 6 or $8 \%$ for JMY and

874 WHAMM, respectively. All other proteins were separated on $10 \%$ gels.

875 Electrophoresis was performed in Laemmli running buffer $(0.1 \%$ SDS, $25 \mathrm{mM}$ Tris,

$876192 \mathrm{mM}$ glycine). Proteins were transferred from gels to nitrocellulose membranes for

$87750 \mathrm{~min}$ at $400 \mathrm{~mA}$ in pre-cooled transfer buffer (192 mM glycine, $25 \mathrm{mM}$ Tris, 10\%

878 methanol). After transfer, membranes were blocked in 5\% milk powder (MP) in Tris-

879 buffered saline (TBS) supplemented with $0.2 \%$ Tween 20 (TBS-TMP) or in 0.2-3\%

880 bovine serum albumin (BSA) for at least $30 \mathrm{~min}$. Primary antibodies were diluted in

881 TBS-TMP or BSA and membranes were incubated for $2 \mathrm{~h}$ at room temperature or

882 overnight at $4^{\circ} \mathrm{C}$. Three washes with TBS supplemented with Tween 20 (TBS-T)

883 were followed by incubation with anti-mouse or -rabbit secondary antibodies

884 conjugated to HRP diluted in TBS-TMP or BSA. Membranes were washed twice with 
885 TBS-T and once with TBS before the signal from HRP-conjugated antibodies was

886 revealed using enhanced chemiluminescence (ECL) or ECL prime and photographic 887 films.

888

889

890 Virus production

891 Production of HPV16 PsVs

892 PsV production was performed as previously described (Buck et al., 2005). A total of $8931.8 \times 10^{7} 293$ TT cells were seeded in $145 \mathrm{~mm}$ cell culture dishes. The next day, cells

894 were co-transfected with p16sheLL and the reporter plasmid pCIneoEGFP (GFP) or $895 \mathrm{pRwB}(\mathrm{RFP})$. Both plasmids (30 $\mu \mathrm{g}$ each) as well as Lipofectamine $2000(132.5 \mu \mathrm{l})$ 896 were diluted in optiMEM and incubated for $5 \mathrm{~min}$ at room temperature. The DNA 897 dilution was added to the Lipofectamine 2000 dilution and samples were incubated 898 for $20 \mathrm{~min}$ at room temperature before the transfection mix was added to fresh 899 growth medium in the dishes. At $48 \mathrm{~h}$ post transfection, cells were harvested and 900 pelleted. For cell lysis and virus maturation, the pellet was incubated with $0.35 \%$ Brij $90158,0.2 \%$ Plasmid Safe DNase and $0.2 \%$ benzonase for $24 \mathrm{~h}$ at $37^{\circ} \mathrm{C}$ on an overhead 902 rotator. PsVs were purified using a linear 25\%-39\% OptiPrep density gradient. A PsV 903 fraction at around 30\% OptiPrep was collected with a needle and analyzed for virus 904 content and purity by Coomassie staining of SDS-PAGE gels.

905

906

Labeling of HPV16 PsVs

907 HPV16 PsVs were incubated with Alexa Fluor 488, 568, 594 or 647 succinimidyl 908 ester in virion buffer (635 mM NaCl, $0.9 \mathrm{mM} \mathrm{CaCl}_{2}, 0.5 \mathrm{mM} \mathrm{MgCl}_{2}, 2.1 \mathrm{mM} \mathrm{KCl}$ in 909 PBS, pH 7.6) using a 1:8 molar ratio of $\mathrm{L} 1$ to the dye for $1 \mathrm{~h}$ on an overhead rotator

910 (Schelhaas et al., 2008; Ventayol and Schelhaas, 2015). Free dye was removed by 
911 ultracentrifugation using a 15-25-39\% OptiPrep step gradient. The labeled virus

912 between the $25 \%$ and $39 \%$ OptiPrep fraction was collected with a needle. The PsV

913 concentration was determined by SDS-PAGE and subsequent Coomassie staining.

914 The labeled virus was characterized by binding to glass coverslips and HeLa ATCC

915 cells (Ventayol and Schelhaas, 2015). Labeling of PsVs with pHrodo was achieved

916 following the same protocol. Virus characterization was performed in citric acid buffer

917 (pH 4.4) (Ventayol and Schelhaas, 2015).

918

919 Infection experiments

920 Infection of KO cells

921 NIH-3T3 $\left(4 \times 10^{5}\right.$ cells/well) and U2OS (both $5 \times 10^{4}$ cells/well) wild type and WASH

922 KO cells were seeded in 12-well plates. The next day, the growth medium was 923 replaced with $300 \mu$ fresh growth medium and HPV16-GFP was added to result in $92420 \%$ infection in wild type cells. The virus was bound on a shaker at $37^{\circ} \mathrm{C}$. At $2 \mathrm{~h}$ p.i., 925 the inoculum was replaced by fresh growth medium and infection was continued. At $92648 \mathrm{~h}$ p.i., cells were trypsinized and fixed in 4\% PFA for $15 \mathrm{~min}$ at room temperature.

927 Cells were resuspended in FACS buffer (250 mM EDTA, 2\% FBS, $0.02 \% \mathrm{NaN}_{3}$ in 928 PBS) and analyzed for infection (percentage of GFP positive cells) by flow cytometry 929 (FACSCalibur, Becton Dickinson). Gating of infected cells was done with help of 930 uninfected controls. The percentage of infected cells was normalized to the 931 respective wild type cells using Microsoft Excel.

932

Inhibitor studies

934 HeLa ATCC cells were seeded in 12-well plates $\left(5 \times 10^{4}\right.$ cells/well) about $16 \mathrm{~h}$ prior

935 to experimentation. Retro-2 experiments were done with HeLa Kyoto cells $\left(1 \times 10^{5}\right.$ 936 cells/well). For HPV16 and HSV-1 infection experiments, small compound inhibitors 
937 and solvent controls were diluted in growth medium, while they were diluted in

938 infection medium (RPMI supplemented with $30 \mathrm{mM}$ HEPES, pH 6.5) for VSV

939 infection. Cells were pre-treated with inhibitors or solvent controls for $30 \mathrm{~min}$ at

940 indicated concentrations and infected with HPV16-GFP as described above. The

941 inoculum was replaced at $2 \mathrm{~h}$ p.i. and infection was continued in presence of the

942 inhibitor. At $12 \mathrm{~h}$ p.i., inhibitors were exchanged for $10 \mathrm{mM} \mathrm{NH}_{4} \mathrm{Cl} / 10 \mathrm{mM} \mathrm{HEPES}$ in

943 growth medium to reduce cytotoxicity (Schelhaas et al., 2012). Cells were fixed and

944 processed for flow cytometry analysis as described above. For infection with VSV-

945 GFP, the virus was added to the infection medium +/- inhibitor to result in $20 \%$

946 infection in solvent treated controls. VSV-GFP was bound for $2 \mathrm{~h}$ on a shaker at $37^{\circ} \mathrm{C}$

947 until the inoculum was replaced with $1 \mathrm{ml}$ growth medium. Cells were trypsinized and

948 fixed at 6 h p.i. as described for HPV16. HSV-1 infection was carried out in growth

949 medium containing the inhibitor or the solvent control. The virus was bound at $4^{\circ} \mathrm{C}$ for

$9501 \mathrm{~h}$, the medium was exchanged for fresh, warm medium with inhibitors and infection

951 was continued for $5 \mathrm{~h}$ at $37^{\circ} \mathrm{C}$. Cells were fixed at $6 \mathrm{~h}$ p.i. as described before.

952

953 To investigate additional effects of retro-2 treatment on HPV16 trafficking, the virus

954 was accumulated in the endolysosomal system by treatment with $10 \mathrm{mM} \mathrm{NH}_{4} \mathrm{Cl}$ for 955 the first $12 \mathrm{~h}$ of infection (Schelhaas et al., 2012). Then, the inhibitors were replaced 956 by $50 / 100 \mu \mathrm{M}$ retro- 2 and cells were fixed at $48 \mathrm{~h}$ p.i. as described above. All 957 samples were analyzed for infection (percentage of GFP positive cells) by flow 958 cytometry (FACSCalibur, Becton Dickinson). Gating of infected cells was done with 959 help of solvent treated controls to which the percentage of infected cells was 960 normalized. 961

962 Infection studies after siRNA-mediated depletion 
963 For siRNA-mediated knockdown, $2 \times 10^{3}$ or $2 \times 10^{4}$ HeLa Kyoto cells were reverse

964 transfected in 96-well optical bottom plates or 12-well plates, respectively.

965 Transfection was performed using $0.2 \mu \mathrm{l}$ (96-well) or $0.5 \mu \mathrm{l}$ (12-well) Lipofectamine

966 RNAi max per well diluted in optiMEM and siRNAs were diluted in optiMEM to reach

967 the working concentration indicated in supplemental table 1 . The following procedure

968 and incubation times were as for Lipofectamine 2000. Besides the siRNA against the

969 cellular proteins of interest, the AllStars negative siRNA (ctrl.) was included as a non-

970 targeting control, whereas the AllStars death siRNA was used to test for successful

971 transfection. Moreover, an siRNA targeting GFP was included to suppress the

972 expression of the HPV16-GFP reporter plasmid as a measure for maximal reduction

973 of infection. For RNAi against WASH, cells were transfected twice in $48 \mathrm{~h}$ intervals.

974 Cells were infected with HPV16-GFP at $48 \mathrm{~h}$ post transfection to result in $20 \%$

975 infection in ctrl. negative transfected controls. In 12-well plates, infection was

976 performed and analyzed by flow cytometry as described above. Absolute infection

977 values were normalized to ctrl. transfected controls. In 96-well plates, the virus was

978 added without prior medium exchange to reduce cell loss. At $48 \mathrm{~h}$ p.i., cells were

979 fixed in 4\% PFA in PBS and nuclei were stained with RedDot2 for 30 min after

980 permeabilization with $0.1 \%$ Triton in PBS. Infection was analyzed by automated

981 microscopy on a Zeiss Axiovert Z.1 microscope equipped with a Yokogawa CSU22

982 spinning disk module (Visitron Systems). Images were acquired using a 20x

983 objective, a CoolSnap HQ camera (Photometrics) and MetaMorph Software. Cell

984 number and infection were determined using a MATLAB-based infection scoring

985 procedure (Engel et al., 2011). The program detects nuclei and infection signal

986 individually, based on their limiting intensity edges. The edges were filled to objects,

987 which were classified by size. Binary masks of nuclei and infection signal were

988 created and cells were classified as infected if equal or greater than $5 \%$ of their 
989 nuclei overlapped with infection signal above a certain threshold. In this study, signal

990 twice above the background in the uninfected sample was considered infected. An

991 infection index was obtained for each image and averaged per well (Snijder et al., 992 2012).

993

994 Infection with VV-GFP was carried out in 96-well plates following the same protocol 995 as for HPV16 with the exception that $3 \times 10^{3}$ cells were transfected. Virus amounts 996 leading to $20 \%$ infection in ctrl. treated controls were used. Cells were fixed at $6 \mathrm{~h} \mathrm{p.i.}$ 997 and analyzed by automated microscopy, as described above.

998

999 For siRNA experiments with SFV, $5 \times 10^{4}$ HeLa Kyoto cells were reverse transfected 1000 in 12-well plates. Infection was performed by addition of the virus to infection medium 1001 (RPMI supplemented with 10\% FBS, 10 mM HEPES (pH 7.3)). The virus was bound 1002 on a shaker at $37^{\circ} \mathrm{C}$. At $2 \mathrm{~h}$ p.i., the inoculum was replaced by growth medium. Cells 1003 were trypsinized and fixed at 6 h p.i.. Since SFV did not carry a fluorescent reporter 1004 plasmid, samples were immunostained for SFV E1/E2 after fixation at $6 \mathrm{~h}$ p.i.. Cells were permeabilized with FACS perm (250 mM EDTA, 2\% FBS, 0.02\% $\mathrm{NaN}_{3}, 0.05 \%$

1006 Saponin in PBS) for 30 min at room temperature and subsequently incubated with 1007 the Lady Di antiserum (Singh and Helenius, 1992) diluted in FACS perm for $2 \mathrm{~h}$ at 1008 room temperature. Samples were washed thrice with FACS perm and incubated with 1009 an anti-rabbit AF488 secondary antibody in FACS perm for $1 \mathrm{~h}$ at room temperature.

1010 Washing with FACS perm was followed by infection scoring with FACS analysis 1011 (FACSCalibur, Becton Dickinson) as described for HPV16. Infection values were 1012 normalized to crtl. using Microsoft Excel.

1013

1014 Infection studies in transiently transfected cells 
1015 NIH-3T3 wild type and WASH KO cells were seeded in 12-well plates

1016 (4 × 10 4 cells/well). One day later, cells were transfected with plasmids $(1 \mu \mathrm{g})$

1017 encoding EGFP-WASH or EGFP, YFP-WASH or YFP-WASH dWCA using

1018 Lipofectamine 2000 (0.5 $\mu \mathrm{l} /$ well) diluted in optiMEM. Incubation times were the same

1019 as for virus preparation. At about $16 \mathrm{~h}$ post transfection, cells were infected with

1020 HPV16-RFP as described above to result in $20 \%$ infection in control cells transfected

1021 with GFP. Cells were trypsinized at $48 \mathrm{~h}$ p.i., fixed in 4\% PFA in PBS and analyzed

1022 by flow cytometry (Guava easyCyte, Merck). Final analysis was performed with

1023 FlowJo. Transfected cells were gated with help of untransfected controls. Then, the

1024 GFP positive population was gated for infection using transfected, but uninfected

1025 controls. The percentage of transfected and infected cells (GFP and RFP positive)

1026 was normalized to NIH-3T3 wild type cells transfected with the GFP control to obtain

1027 relative infection values using Microsoft Excel.

1028

1029 HeLa ATCC cells were transfected with Scar-W and -WA constructs as described

1030 above 16-24 $\mathrm{h}$ prior to infection. Cells were infected with HPV16-RFP and fixed $48 \mathrm{~h}$

1031 p.i. using 4\% PFA. Infection was scored using an Olympus IX70 inverted microscope

1032 equipped with an electron multiplier CCD camera (EDMCCD, C9100-02, Hamamatsu

1033 Photonics K. K.) and a monochromator for epifluorescence excitation. Images were

1034 thresholded manually and at least 100 cells were scored for transfection and infection

1035 using Fiji.

1036

1037 HPV16 binding assay

1038 For analysis of HPV16 binding by flow cytometry, $5 \times 10^{4} \mathrm{NIH}-3 \mathrm{~T} 3$ wild type and

1039 WASH KO cells were seeded per well of a 12-well plate. The next day, fluorescently 1040 labeled HPV16-AF488 ( 1000 particles/cell) was bound to the cells for $2 \mathrm{~h}$ on a 
1041 shaker at $37^{\circ} \mathrm{C}$. Cells treated with siRNAs were reseeded at $48 \mathrm{~h}$ post transfection (5

$1042 \times 10^{4}$ cells/well). Virus binding was performed once cells were attached, typically

1043 about $6 \mathrm{~h}$ post seeding. As a non-binding control, HPV16-AF488 was pre-incubated

1044 with $1 \mathrm{mg} / \mathrm{ml}$ heparin for $1 \mathrm{~h}$ at room temperature prior to binding to cells (Cerqueira

1045 et al., 2013). Cells were trypsinized and fixed with 4\% PFA. Virus binding was

1046 analyzed by measuring the mean fluorescence intensity (geometric mean) of cells in

1047 flow cytometry (FACSCalibur). The geometric mean of uninfected cells was

1048 subtracted from infected cells and virus binding was normalized to control cells. A

1049 similar procedure was applied to measure virus binding to HeLa Kyoto cells depleted

1050 of SNX2. Binding was qualitatively assessed for NIH-3T3 wild type and WASH KO

1051 cells as well as after SNX2 KD in HeLa Kyoto cells. For this, HPV16-AF568 was

1052 bound to these cells as described above. At $2 \mathrm{~h}$ p.i., cells were fixed with $4 \%$ PFA

1053 and stained with $0.1 \mu \mathrm{g} / \mathrm{ml}$ phalloidin-Atto488 diluted in PHEM buffer (60 mM PIPES,

105410 mM EGTA, 2 mM MgCl 2,25 mM HEPES, pH 6.9) supplemented with $0.01 \%$ Triton

$1055 \mathrm{X}-100$ for $30 \mathrm{~min}$. Cells were washed thrice with PBS and mounted on glass slides

1056 using AF1 mounting medium. Images were acquired with a Zeiss Axiovert Z.1

1057 microscope equipped with a Yokogawa CSU22 spinning disk module (Visitron

1058 Systems) using a 40x objective, a CoolSnap HQ camera (Photometrics) and

1059 MetaMorph Software. Z-stacks covering the cell volume were converted to maximum

1060 intensity projections using Fiji. Brightness and contrast were adjusted using

1061 uninfected samples.

1062

1063 For microscopy-based analysis of retro-2 treated cells, HeLa Kyoto cells were 1064 seeded to confluency in a 96-well plate. At least 6 h post seeding, HPV16-AF594 or -

1065 AF488 ( 1000 particles/cell) were bound to cells in presence or absence of the 1066 inhibitor, as above. At $2 \mathrm{~h}$ p.i., cells were fixed with 4\% PFA and binding was 
1067 analyzed using a 40x objective of a spinning disk confocal microscope described

1068 above. Z-stacks covering the cell volume were converted to sum intensity z-

1069 projections using Fiji. The mean fluorescence intensities of HPV16 AF were

1070 measured as arbitrary units per field of view. Background intensities measured in

1071 uninfected samples were subtracted from raw intensities, which were normalized to

1072 solvent treated control cells.

1073

1074

1075

1076

Infectious internalization assay

1077 One day prior to infection, $5 \times 10^{4}$ HeLa Kyoto cells were seeded per well of a 12-well

1078 plate. Cells were pre-incubated with inhibitors and infected with HPV16-GFP as

1079 described above. At 12 h p.i., extracellular virus was inactivated by washing with $1 \mathrm{ml}$

$10800.1 \mathrm{mM}$ CAPS buffer $(\mathrm{pH}$ 10.5) for $90 \mathrm{sec}$ (Schelhaas et al., 2012; Becker et al.,

1081 2018). The cells were washed twice with PBS to remove CAPS and infection was

1082 continued in fresh growth medium. To control for inhibitor reversibility, the inhibitor

1083 was washed out thrice with PBS without prior CAPS treatment and fresh growth

1084 medium was added. At 48 h p.i., cells were fixed and infection was scored by flow

1085 cytometry as described above. Infection results were normalized to inhibitor

1086 reversibility.

1087

1088 Endocytosis assay with HPV16-pHrodo

1089 Cells were reverse transfected with siRNAs as described above. At $48 \mathrm{~h}$ after the first

1090 (Arp3, SNX2) or second (WASH) siRNA transfection, HPV16-pHrodo ( 1000

1091 particles/cell) was added to $350 \mu \mathrm{l}$ growth medium and bound for $2 \mathrm{~h}$ on a shaker.

1092 The inoculum was replaced at $2 \mathrm{~h}$ p.i. and cells were imaged live at $6 \mathrm{~h}$ p.i. at $37^{\circ} \mathrm{C}$ 
1093 and $5 \% \mathrm{CO}_{2}$ in humidified atmosphere using custom made imaging chambers and

1094 DMEM high glucose without phenol red supplemented with $10 \%$ FBS, $1 \%$ L1095 glutamine and 1\% penicillin/streptomycin. Images were acquired on a Zeiss Axiovert

1096 Z.1 microscope equipped with a Yokogawa CSU22 spinning disk module (Visitron

1097 Systems) using a 40x objective, a CoolSnap HQ camera (Photometrics) and

1098 MetaMorph Software. Average intensity projections of confocal slices were generated

1099 using Fiji software. Intensity based analysis was performed with CellProfiler (Becker

1100 et al., 2018). In brief, the virus signal was enhanced by application of a white top-hat

1101 filter. Virus spots were segmented by application of a gaussian filter and maximum

1102 correlation thresholding. Virus intensity was then measured in enhanced and original

1103 images. Pivot tables (Microsoft Excel) were used to summarize the intensity of spots

1104 per condition. These values were normalized to the cell number, which was

1105 determined by manual counting from brightfield images. The virus intensity per cell

1106 was then normalized to ctrl. treated controls to obtain relative internalization values.

1107 Cell outlines were created manually for presentation purposes.

1108

1109 Electron microscopy and CLEM

1110 CLEM

1111 A circular mark used for localization of unroofed cells was generated in the center of

1112 coverslips (22 mm diameter) using a diamond knife. For ECM production, $2 \times 10^{6}$

1113 HaCaT cells were seeded onto the coverslips placed in a 6-well plate. At $48 \mathrm{~h}$ post

1114 seeding, ECM was obtained by detaching cells through incubation with $10 \mathrm{mM}$

1115 EDTA/EGTA for $45 \mathrm{~min}$ at $37^{\circ} \mathrm{C}$, subsequent clapping of the plate and washes with

1116 PBS (Culp et al., 2006). HPV16-AF568 was bound to the ECM in $1 \mathrm{ml}$ growth

1117 medium/well for $2 \mathrm{~h}$ on a shaker at $37^{\circ} \mathrm{C}$. HaCaT cells were trypsinized and $12 \times 10^{5}$

1118 cells/well were seeded onto the virus bound to ECM. At $1 \mathrm{~h}$ post seeding, $10 \mu \mathrm{g} / \mathrm{ml}$ 
1119 cytoD or DMSO (solvent control) were added. A total of $6 \mathrm{~h}$ post seeding, cells were

1120 put on ice and washed thrice with cold stabilization buffer $(70 \mathrm{mM} \mathrm{KCl}, 30 \mathrm{mM}$

1121 HEPES ( $\mathrm{pH} 7.4$ with $\mathrm{KOH}$ ), $5 \mathrm{mM} \mathrm{MgCl}_{2}$ ). For unroofing, the cells were kept on ice

1122 and $1 \mathrm{ml}$ cold $2 \%$ PFA in stabilization buffer was aspirated with a $1 \mathrm{ml}$ pipette. The

1123 pipette was positioned above the marked area in the center of the coverslip and PFA

1124 was harshly released onto the cells. The coverslip was then rapidly transferred to a

1125 new well containing cold $2 \%$ PFA in stabilization buffer to avoid sedimentation of cell

1126 debris on the unroofed membrane. Membrane sheets were fixed for 10 min at $4^{\circ} \mathrm{C}$.

1127 Samples were mounted in custom-made imaging chambers and imaged in PBS at a

1128 Nikon Ti Eclipse microscope equipped with a PerkinElmer UltraVIEW VoX spinning

1129 disk module. Images were acquired using a 60x objective, an Orca Flash 4 camera

1130 (Hamamatsu) and Volocity software (PerkinElmer, version 6.3). Montages of $10 \times 10$

1131 images and $10 \%$ overlap were acquired around the center of the marked area. The

1132 unroofed membranes were prepared for EM by fixation with $2 \%$ glutaraldehyde (GA)

1133 in PBS overnight at $4^{\circ} \mathrm{C}$. After two washes with water, samples were incubated with

$11340.1 \%$ tannic acid for $20 \mathrm{~min}$ at room temperature and subsequently washed with

1135 water. Contrasting was performed with $0.1 \%$ uranyl acetate (UAC) for 20 min at room

1136 temperature. After three washes with water, samples were dehydrated with a series

1137 of ethanol solutions $(15 \% / 30 \% / 50 \% / 70 \% / 80 \% / 90 \% / 100 \%)$. Coverslips were

1138 incubated with each solution for $5 \mathrm{~min}$, incubation with $100 \%$ ethanol was repeated

1139 thrice. Samples were dried using hexamethyldisilazane (HDMS). After 5 min

1140 incubation at room temperature, fresh HDMS was added and samples were

1141 incubated for further $30 \mathrm{~min}$ at room temperature. Coverslips were dried and coated

1142 under vacuum using a Balzers BAF301 device (former Balzers AG, Liechtenstein). A

1143 first layer of platinum was applied at an angle of $11^{\circ} \mathrm{C}$ while rotating. A second layer

1144 of carbon was applied at an angle of $90^{\circ}$ while rotating. Coverslips were cut to fit on 
1145 EM grids before 5\% hydrofluoric acid were used to separate the metal replica from

1146 the glass. Replicas were extensively washed with water prior to transfer to glow

1147 discharged, formvar coated EM grids. Replicas were imaged with a phase contrast

1148 microscope for orientation. Intact membranes associated with virus particles were

1149 manually selected based on overlays of images from fluorescence and phase

1150 contrast microscopy and imaged at a TEM (Jeol JEM-1400, Jeol Ltd., Tokyo, Japan,

1151 camera: TemCam F416; TVIPS, Gauting, Germany). Membrane sheets were imaged

1152 using montages of $5 \times 5$ images and $15 \%$ overlap. Fluorescence and EM images

1153 were initially overlaid manually using Photoshop, then the Fiji plugin Landmark

1154 Correspondences (Saalfeld and Tomancák, 2008) was used for transformation of the

1155 fluorescence image according to the EM image using three manually identified

1156 landmarks. For analysis, HPV16 was identified manually based on the fluorescent

1157 signal and classification was done by visual evaluation of associated structures in

1158 TEM images. At total of 134 and 101 membrane associated virus particles in DMSO

1159 (7 membranes) and cytoD (5 membranes) treated cells were analyzed, respectively.

1160

1161 Ultra-thin section EM

1162 Samples for ultra-thin section EM were prepared and analyzed as described

1163 previously (Bannach et al., 2020). A total of $1-2 \times 10^{5} \mathrm{NIH} 3 \mathrm{~T} 3$ wild type, WASH KO

1164 and HeLa Kyoto cells or were seeded in $3 \mathrm{~cm}$ dishes. Two days post seeding, cells

1165 were either pretreated with inhibitors for 30 min or were left untreated. Cells were

1166 infected with $40 \mu \mathrm{g} \mathrm{HPV} 16$ PsVs in $1 \mathrm{ml}$ growth medium. At $6 \mathrm{~h}$ p.i., cells were fixed

1167 in $2.5 \%$ GA in PBS ( $\mathrm{pH} \mathrm{7.2)} \mathrm{for} 10 \mathrm{~min}$ at room temperature. A second fixation was

1168 performed with cold $2.5 \% \mathrm{GA}$ at $4^{\circ} \mathrm{C}$ overnight. Cells were washed thrice with PBS,

1169 post-fixed with $1 \% \mathrm{OsO}_{4}$ in $\mathrm{ddH}_{2} \mathrm{O}$ for $1 \mathrm{~h}$ and washed twice with $\mathrm{ddH}_{2} \mathrm{O}$ at room

1170 temperature and at $4^{\circ} \mathrm{C}$ for $20 \mathrm{~min}$, respectively. Block contrasting was performed 
1171 with $0.5 \% \mathrm{UAC}$ in $\mathrm{ddH}_{2} \mathrm{O}$ at $4^{\circ} \mathrm{C}$ overnight. Cells were washed thrice with $\mathrm{ddH}_{2} \mathrm{O}$ and

1172 dehydrated using ascending graded alcohol series. Detaching and dehydrating with

1173 propylene oxide were followed by incubation with propylene oxide and epoxy resin

$1174(1: 3,1: 1,3: 1)$ for $2 \mathrm{~h}$ each, before cells were incubated with pure epoxy resin for $3 \mathrm{~h}$

1175 and embedded in BEEM capsules. The resin was allowed to polymerize at $60^{\circ} \mathrm{C}$ for

1176 three days before $60 \mathrm{~nm}$ ultra-thin sections were cut and counterstained with uranyl

1177 acetate and lead. Samples were imaged at a $80 \mathrm{kV}$ on a Tecnai 12 electron

1178 microscope (FEI) using an Olympus Veleta 4k CCD camera or Ditabis imaging

1179 plates. Images were contrast enhanced and cropped using Adobe Photoshop CS4.

1180 The total number of endocytic pits per cell was determined for 31 and 43 cells in wild

1181 type and WASH KO cells, respectively, in two independent experiments. Only

1182 endocytic pits containing virus(es) were counted, since HPV16 pits are hardly

1183 distinguishable from uncoated pits from other endocytic pathways without further

1184 staining. Pit numbers were normalized to wild type cells.

1185

1186 Immunogold labeling

1187 HeLa ATCC cells $\left(2-3 \times 10^{5}\right.$ cells $)$ were seeded in $6 \mathrm{~cm}$ dishes. The next day, cells

1188 were transfected with a plasmid encoding EGFP-WASH or EGFP-SNX2 $(7 \mu \mathrm{g})$ using

1189 Lipofectamine $2000(3.5 \mu \mathrm{l})$ according to the manufacturer's instructions or left

1190 untransfected. At $48 \mathrm{~h}$ post transfection, cells were infected with $80 \mu \mathrm{g}$ HPV16 PsVs

1191 and incubated for $6 \mathrm{~h}$ at $37^{\circ} \mathrm{C}$ until pre-fixation by addition of $4 \%$ formaldehyde in

$11920.1 \mathrm{M}$ phosphate buffer $(\mathrm{pH} 7.2)$ to the culture medium (1:1 ratio) for $5 \mathrm{~min}$. Then,

1193 cells were fixed in $2 \%$ formaldehyde, and $0.2 \%$ GA in $0.1 \mathrm{M}$ phosphate buffer

1194 (pH 7.2). Samples were processed for TEM as previously described (Humbel and

1195 Stierhof, 2009). In brief, cells were quenched by incubation in $0.1 \%$ glycine in $0.1 \mathrm{M}$

1196 PB (2 x $30 \mathrm{~min})$, washed thrice in 0.1 M PB for 30 min and scraped with $1 \%$ gelatin. 
1197 After centrifugation, the gelatin was replaced with $12 \%$ gelatin and cells were infused

1198 at $37^{\circ} \mathrm{C}$. Cells were cooled down on ice and the gelatin-cell pellet was cut into small

1199 blocks that were infused with $2.3 \mathrm{M}$ sucrose overnight at $4^{\circ} \mathrm{C}$. The blocks were

1200 mounted on specimen carriers and frozen in liquid nitrogen. Ultra-thin cryosections

1201 were prepared according to Tokuyasu (Tokuyasu, 1980). In brief, ultra-thin

1202 cryosections of $50 \mathrm{~nm}$ thickness were prepared with an EM UC6/FC6 ultramicrotome

1203 (Leica Microsystems). Sections were collected in a sucrose-methylcellulose mixture

1204 and stored on TEM grids at $4^{\circ} \mathrm{C}$ until further processing. Methylcellulose was melted

1205 and sections were washed five times with $20 \mathrm{mM}$ glycine in PBS. Quenching was

1206 followed by blocking with 1\% BSA for 3 min. Cells were then incubated with a GFP-

1207 antibody for 30 min, washed 6 times with $0.1 \%$ BSA in PBS and incubated with

1208 protein A gold $15 \mathrm{~nm}$ for 20 min. Sections were rinsed 10 times with PBS and re-fixed

1209 in 1\% GA in PBS (pH 7.2). For double labeling, the sections were quenched, blocked

1210 and immunostained with an actin antibody as described above. Then sections were

1211 incubated with a rabbit anti-mouse bridging antibody followed by 6 washes with $0.1 \%$

1212 BSA in PBS and incubated with protein A gold $10 \mathrm{~nm}$ (1:50). Sections were rinsed

121310 times with PBS and re-fixed in 1\% GA in PBS, pH 7.2. Sections were rinsed 10

1214 times with dd $\mathrm{H}_{2} \mathrm{O}$ and contrasted with uranyl acetate for 6 min $(\mathrm{pH} 7)$. After one wash

1215 with $\mathrm{ddH}_{2} \mathrm{O}$, cells were embedded in an uranyl acetate-methylcellulose mixture $(\mathrm{pH}$

1216 4) for $10 \mathrm{~min}$. After looping out with filter paper, sections were dried and images were

1217 acquired as above.

1218

1219 Fluorescence microscopy

1220 CLC and virus internalization analysis by live cell TIRF microscopy

1221 HeLa ATCC cells were seeded on coverslips in 12-well plates ( $5 \times 10^{4}$ cells/well) one 1222 day prior to transfection. Cells were transfected with plasmids encoding lifeact-EGFP, 
1223 EGFP-WASH, EGFP-SNX2, mRFP-CLC or EGFP-Dyn2 as described above. For 1224 internalization analysis, fluorescently labeled virus (HPV16-AF594/AF647) was

1225 bound at $37^{\circ} \mathrm{C}$ at about $18 \mathrm{~h}$ post transfection. At $1 \mathrm{~h}$ p.i., coverslips were mounted in 1226 custom-made imaging chambers. Cells were imaged at $37^{\circ} \mathrm{C}$ and $5 \% \mathrm{CO}_{2}$ in 1227 humidified atmosphere in DMEM without phenol red supplemented with $10 \%$ FBS, $12281 \%$ L-glutamine and $1 \%$ penicillin/streptomycin. Time lapse movies of cells 1229 expressing lifeact-EGFP were acquired with a 60x TIRF-objective on an Olympus 1230 IX70 microscope equipped with a TIRF condenser and an electron multiplier CCD 1231 camera (EDMCCD, C9100-02, Hamamatsu Photonics K. K.) using MetaMorph 1232 software (Molecular Devices) (Visitron Systems). All other time lapse movies were 1233 acquired using a 100x TIRF-objective at an Olympus IX83 microscope equipped with 1234 a four-line TIRF condenser and an EMCCD camera (iXon Ultra 888, Andor Oxford 1235 Instruments) using CellSens Dimensions software (Olympus). Movies were acquired 1236 with $0.5 \mathrm{~Hz}$ frame rate for $5 \mathrm{~min}$. HPV16 entry events were identified manually and 1237 the intensity of fluorescent proteins at virus entry sites was analyzed after rolling ball 1238 background subtraction and filtering (mean intensity filter) with Fiji. Kymographs, 1239 intensity profiles along a manually drawn line through the virus/clathrin signal over 1240 time, were generated with Fiji after background subtraction and filtering. Intensity 1241 profiles measured with Fiji using a circular region of interest, were processed by $1242 \mathrm{~min} / \mathrm{max}$ normalization and aligned by setting the half time of virus internalization to 0 1243 s. Profiles were plotted with Microsoft Excel. Moving averages of signals are shown 1244 as a trendline (period 4-20). The time points of recruitment onset, maximal signal or 1245 signal loss were manually determined relative to the half time of internalization and 1246 box plots were generated with GraphPad Prism. Cells co-transfected with CLC and 1247 Dyn2 were analyzed the same way at about $16 \mathrm{~h}$ post transfection. Movies were 1248 compressed to 20 fps and PNG. 
1250 Proximity ligation assay

1251 For ECM production on coverslips, $5 \times 10^{5} \mathrm{HaCaT}$ cells were seeded per well of a 1252 12-well plate and cultivated at $37^{\circ} \mathrm{C}$ for 2 days. Additionally, $7 \times 10^{4} \mathrm{HaCaT}$ cells 1253 were seeded per well and transfected the next day with a plasmid encoding HA1254 CD151. The procedure was the same as for infection studies with transfected cells, 1255 but $0.4 \mu \mathrm{g}$ DNA and $1 \mu \mathrm{l}$ Lipofectamine 2000 per well were used. ECM on coverslips 1256 was obtained by detaching cells with $0.5 \mathrm{ml} 10 \mathrm{mM}$ EDTA/EGTA as described above.

1257 HPV16-AF488 ( 1000 particles/cell) was bound to the ECM in $400 \mu$ growth medium 1258 on a shaker at $37^{\circ} \mathrm{C}$. At $2 \mathrm{~h}$ post binding, HaCaT cells expressing HA-CD151 were 1259 trypsinized and transferred onto the virus bound to ECM. Cells were allowed to 1260 attach for $5 \mathrm{~h}$ and fixed in $2 \%$ PFA in PBS for $10 \mathrm{~min}$ at $4^{\circ} \mathrm{C}$. Cells were 1261 permeabilized with $0.2 \%$ Brij 58 in PBS for $10 \mathrm{~min}$ at room temperature prior to 1262 blocking in $1 \% \mathrm{BSA}$ in PBS for $30 \mathrm{~min}$ at room temperature. Primary antibody 1263 staining against HA (1:10,000) and WASH (1:500) was carried out in a wet chamber 1264 overnight at $4^{\circ} \mathrm{C}$. Next, cells were incubated with anti-mouse PLUS and anti-rabbit 1265 MINUS Duolink PLA probes diluted 1:5 in $1 \%$ BSA in PBS for $1 \mathrm{~h}$ at $37^{\circ} \mathrm{C}$ in a 1266 humidity chamber. Duolink In Situ Detection Reagents Red were used for further 1267 sample processing. For ligation of PLA probes, $1.25 \mathrm{U}$ ligase per sample were added 1268 to the corresponding ligation buffer diluted in $d d \mathrm{H}_{2} \mathrm{O}$. The cells were incubated in at 1269 wet chamber for $30 \mathrm{~min}$ at $37^{\circ} \mathrm{C}$ followed by two washes with wash buffer B (2 min, 1270 room temperature). Amplification was performed with $6.25 \mathrm{U}$ polymerase and Duolink 1271 Amplification Red (1:5) diluted in $\mathrm{ddH}_{2} \mathrm{O}$ in a wet chamber at $37^{\circ} \mathrm{C}$ for 100 min. Cells 1272 were washed twice for $10 \mathrm{~min}$ with wash buffer $\mathrm{B}$ at room temperature followed by a 1273 quick wash with $0.1 \times$ wash buffer $B$ in $d_{d d H_{2}} \mathrm{O}$. As a counterstain, $\mathrm{HA}$ (CD151) was 1274 detected with an anti-mouse AF647 antibody, which was diluted 1:2000 in 1\% BSA in 
1275 PBS. Samples were transferred to custom made imaging chambers. Images were

1276 acquired with a 100x TIRF-objective at an Olympus IX83 microscope equipped with a

1277 four-line TIRF condenser and an EMCCD camera (iXon Ultra 888, Andor Oxford

1278 Instruments) using CellSens Dimension software (Olympus).

1279

1280 Quantification and statistical analysis

1281 Information on data representation (mean \pm SEM) and $n$ can be found in the figure

1282 legends. Statistical significance was determined using unpaired t-tests conducted

1283 with GraphPad Prism. A P-value below $0.05(P<0.05)$ indicated a significant

1284 difference, denoted by asterisks in the figures ( ${ }^{*} P \leq 0.05,{ }^{* *} P \leq 0.01,{ }^{* \star *} P \leq 0.001$ ).

1285 If not specifically indicated, differences were not significant.

1286 


\section{Supplemental movie legends}

1288 Supplemental movie 1 Actin polymerization coincided with HPV16 internalization

1289 (Related to Figure 2)

1290 HeLa ATCC cells transfected with lifeact-EGFP (actin, green) were infected with

1291 HPV16-AF594 (HPV16, red) and imaged by live cell TIRF-M at $1 \mathrm{~h}$ p.i.. Movies were

1292 acquired with $0.5 \mathrm{~Hz}$ frame rate for $5 \mathrm{~min}$ and processed by background subtraction

1293 and mean filtering. Shown is the virus entry event characterized in Figure 2. Depicted

1294 are 200 frames corresponding to $1: 40$ min real time at a compression rate of 20

1295 frames per seconds (fps). The virus internalizing during the movie is highlighted by a 1296 circle. Scale bar is $200 \mathrm{~nm}$.

1298 Supplemental movie 2 Dynamin recruitment during CME resembled actin 1299 dynamics during HPV16 endocytosis (Related to Figure S1)

1300 HeLa ATCC cells co-transfected with mRFP-clathrin light chain (CLC, red) and

1301 EGFP-dynamin 2 (Dyn2, green) were imaged by live cell TIRF-M. Movies, acquired 1302 with $0.5 \mathrm{~Hz}$ frame rate for $5 \mathrm{~min}$, were processed by background and mean filtering.

1303 Shown are 1:40 min (200 frames) of the CME event characterized in Figure S1 at a 1304 compression rate of $20 \mathrm{fps}$. The CLC of interest is marked by a circle. Scale bar is $1305200 \mathrm{~nm}$.

1307 Supplemental movie 3 WASH co-internalized with HPV16 (Related to Figure 4)

1308 EGFP-WASH (WASH, green) was expressed in HeLa ATCC cells, which were 1309 subsequently infected with HPV16-AF647 (HPV16, red). Movies were acquired by 1310 live cell TIRF-M at $1 \mathrm{~h}$ p.i. for $5 \mathrm{~min}$ at $0.5 \mathrm{~Hz}$ frame rate. The virus entry event 1311 highlighted by a circle was characterized in Figure 4. Shown are 1:14 min 
1312 (150 frames) with a compression rate of 20 frames per second. The movie was

1313 processed by background subtraction and mean filtering. Scale bar is $200 \mathrm{~nm}$.

1314

1315 Supplemental movie 4 SNX2 co-internalized with HPV16 (Related to Figure 6)

1316 HeLa ATCC cells were transfected with EGFP-SNX2 (SNX2, green) and infected

1317 with HPV16-AF647 (HPV16, red). Movies of live cells were acquired with $0.5 \mathrm{~Hz}$

1318 frame rate for 5 min at a TIRF-M. An excerpt of 1:14 min (150 frames) compressed to

131920 frames per second is shown after background subtraction and mean filtering. The

1320 virus particle of interest is marked with a circle. Scale bar is $200 \mathrm{~nm}$.

1321

1322 Supplemental table 1 (siRNA)

\begin{tabular}{|l|l|l|}
\hline siRNA & working concentration (nM) & Qiagen identifier \\
\hline AllStars death & 10 & SI04381048 \\
\hline AllStars negative (siCtrl.) & $10 / 20 / 50$ & SI03650318 \\
\hline Arp3 siRNA_1 & 10 & Hs_ACTR3_5 \\
\hline Arp3 siRNA_2 & 10 & Hs_ACTR3_8 \\
\hline CtBP1 siRNA_1 & 10 & Hs_CTBP1_5 \\
\hline CtBP1 siRNA_2 & 10 & Hs_CTBP1_6 \\
\hline Formin 1 siRNA_1 & 10 & Hs_FMN1_5 \\
\hline Formin 1 siRNA_2 & 10 & Hs_FMN1_6 \\
\hline Formin 1 siRNA_3 & 10 & Hs_FMN1_7 \\
\hline Formin 2 siRNA_1 & 10 & Hs_FMN2_12 \\
\hline Formin 2 siRNA_2 & 10 & Hs_FMN2_7 \\
\hline Formin 2 siRNA_3 & 10 & Hs_FMN2_9 \\
\hline FNL2 siRNA_1 & 10 & Hs_FMNL2_6 \\
\hline FNL2 siRNA_2 & 10 & Hs_FMNL2_7 \\
\hline FNL2 siRNA_3 & 10 & Hs_FMNL2_8 \\
\hline FNL3 siRNA_1 & 10 & Hs_FMNL3_1 \\
\hline FNL3 siRNA_2 & 10 & Hs_FMNL3_5 \\
\hline FNL3 siRNA_3 & 10 & Hs_FMNL3_6 \\
\hline GFP siRNA & 10 & SI04380467 \\
\hline JMY siRNA_1 & 50 & Hs_JMY_1 \\
\hline JMY siRNA_2 & 50 & Hs_JMY_5 \\
\hline DIAPH2 siRNA_1 & 10 & Hs_DIAPH2_1 \\
\hline
\end{tabular}




\begin{tabular}{|l|l|l|}
\hline DIAPH2 siRNA_2 & 10 & Hs_DIAPH2_4 \\
\hline DIAPH2 siRNA_3 & 10 & Hs_DIAPH2_6 \\
\hline N-WASP siRNA_1 & 10 & Hs_WASL_1 \\
\hline N-WASP siRNA_2 & 10 & Hs_WASL_6 \\
\hline SNX2 siRNA_1 & 10 & Hs_SNX2_1 \\
\hline SNX2 siRNA_2 & 10 & Hs_SNX2_3 \\
\hline WASH siRNA_1 & 20 & Hs_WASH1_4 \\
\hline WASH siRNA_2 & 20 & Hs_WASH1_8 \\
\hline WAVE1 siRNA_1 & 20 & Hs_WASF1_3 \\
\hline WAVE1 siRNA_2 & 50 & Hs_WASF1_4 \\
\hline WAVE2 siRNA_1 & 10 & Hs_WASF2_5 \\
\hline WAVE2 siRNA_2 & 10 & Hs_WASF2_6 \\
\hline WHAMM siRNA_1 & 50 & Hs_WHDC1_1 \\
\hline WHAMM siRNA_2 & 50 & Hs_WHDC1_2 \\
\hline Vps26 siRNA_1 & 10 & Hs_VPS26A_1 \\
\hline Vps26 siRNA_2 & 10 & Hs_VPS26A_2 \\
\hline Vps29 siRNA_1 & 10 & Hs_VPS29_6 \\
\hline Vps29 siRNA_2 & 10 & Hs_VPS29_2 \\
\hline
\end{tabular}




\section{References}

1325 Aksoy, P., Gottschalk, E. Y. and Meneses, P. I. (2017) 'HPV entry into cells',

1326 Mutation Research - Reviews in Mutation Research. Elsevier B.V., 772, pp. 13-22.

1327 doi: 10.1016/j.mrrev.2016.09.004.

1328 Aydin, I. et al. (2014) 'Large scale RNAi reveals the requirement of nuclear envelope

1329 breakdown for nuclear import of human papillomaviruses.', PLoS pathogens, 10(5),

1330 p. e1004162. doi: 10.1371/journal.ppat.1004162.

1331 Bannach, C. et al. (2020) 'Epidermal growth factor receptor and Abl2 kinase regulate

1332 distinct steps of Human papillomavirus type 16 endocytosis.', Journal of virology,

1333 2(March). doi: 10.1128/JVI.02143-19.

1334 Becker, M. et al. (2018) 'Extracellular conformational changes in the capsid of human

1335 papillomaviruses contribute to asynchronous uptake into host cells', Journal of

1336 Virology, 92(11), p. JVI.02106-17. doi: 10.1128/jvi.02106-17.

1337 Bement, W. M., Sokac, A. M. and Mandato, C. A. (2003) 'Four-dimensional imaging

1338 of cytoskeletal dynamics in Xenopus oocytes and eggs', Differentiation, 71(9-10), pp.

1339 518-527. doi: 10.1111/j.1432-0436.2003.07109005.x.

1340 Bienkowska-Haba, M. et al. (2012) 'Cyclophilins facilitate dissociation of the human

1341 papillomavirus type 16 capsid protein L1 from the L2/DNA complex following virus

1342 entry.', Journal of virology, 86(18), pp. 9875-87. doi: 10.1128/JVI.00980-12.

1343 Borradori, L. and Sonnenberg, A. (1999) 'Structure and function of

1344 hemidesmosomes: More than simple adhesion complexes', Journal of Investigative

1345 Dermatology, 112(4), pp. 411-418. doi: 10.1046/j.1523-1747.1999.00546.x.

1346 Boukamp, P. et al. (1988) 'Normal keratinization in a spontaneously immortalized

1347 aneuploid human keratinocyte cell line.', The Journal of cell biology, 106(3), pp. 761-

1348 71. doi: 10.1083/jcb.106.3.761.

1349 Brockman, W. W. and Nathans, D. (1974) 'The isolation of simian virus 40 variants

1350 with specifically altered genomes', Proceedings of the National Academy of Sciences

1351 of the United States of America, 71(3), pp. 942-946. doi: 10.1073/pnas.71.3.942.

1352 Bucher, D. et al. (2018) 'Clathrin-Adaptor ratio and membrane tension regulate the

1353 flat-To-curved transition of the clathrin coat during endocytosis', Nature

1354 Communications, 9(1), pp. 1-13. doi: 10.1038/s41467-018-03533-0.

1355 Buck, C. B. et al. (2005) 'Generation of HPV pseudovirions using transfection and

1356 their use in neutralization assays.', Methods in molecular medicine, 119, pp. 445-62.

1357 doi: 10.1385/1-59259-982-6:445. 
1358 Buck, C. B. and Thompson, C. D. (2007) 'Production of Papillomavirus-Based Gene

1359 Transfer Vectors', Current Protocols in Cell Biology, 37(1), pp. 26.1.1-26.1.19. doi:

$136010.1002 / 0471143030 . c b 2601 s 37$.

1361 Bujny, M. V et al. (2007) 'The retromer component sorting nexin-1 is required for

1362 efficient retrograde transport of Shiga toxin from early endosome to the trans Golgi

1363 network.', Journal of cell science, 120, pp. 2010-2021. doi: 10.1242/jcs.003111.

1364 Campellone, K. G. et al. (2008) 'WHAMM Is an Arp2/3 Complex Activator That Binds

1365 Microtubules and Functions in ER to Golgi Transport', Cell, 134(1), pp. 148-161. doi:

1366 10.1016/j.cell.2008.05.032.

1367 Cao, H., Garcia, F. and Mcniven, M. A. (1998) 'Differential Distribution of Dynamin

1368 Isoforms in Mammalian Cells', Molecular Biology of the Cell, 9(September), pp.

1369 2595-2609. doi: 10.1091/mbc.9.9.2595.

1370 Carlton, J. G. et al. (2005) 'Sorting nexin-2 is associated with tubular elements of the

1371 early endosome, but is not essential for retromer-mediated endosome-to-TGN

1372 transport.', Journal of cell science, 118(Pt 19), pp. 4527-39. doi: 10.1242/jcs.02568.

1373 Cerqueira, C. et al. (2013) 'Heparin increases the infectivity of Human Papillomavirus

1374 Type 16 independent of cell surface proteoglycans and induces L1 epitope

1375 exposure', Cellular Microbiology, 15(11), pp. 1818-1836. doi: 10.1111/cmi.12150.

1376 Cerqueira, C. et al. (2015) 'Kallikrein-8 Proteolytically Processes Human

1377 Papillomaviruses in the Extracellular Space To Facilitate Entry into Host Cells.',

1378 Journal of virology, 89(14), pp. 7038-52. doi: 10.1128/JVI.00234-15.

1379 Chadda, R. et al. (2007) 'Cholesterol-sensitive Cdc42 activation regulates actin

1380 polymerization for endocytosis via the GEEC pathway', Traffic, 8(6), pp. 702-717.

1381 doi: 10.1111/j.1600-0854.2007.00565.x.

1382 Collins, A. et al. (2011) 'Structural organization of the actin cytoskeleton at sites of

1383 clathrin-mediated endocytosis', Current Biology, 21(14), pp. 1167-1175. doi:

1384 10.1016/j.cub.2011.05.048.

1385 Culp, T. D. et al. (2006) 'Keratinocyte-Secreted Laminin 5 Can Function as a

1386 Transient Receptor for Human Papillomaviruses by Binding Virions and Transferring

1387 Them to Adjacent Cells', Journal of Virology, 80(18), pp. 8940-8950. doi:

1388 10.1128/jvi.00724-06.

1389 Cureton, D. K. et al. (2009) 'Vesicular Stomatitis Virus Enters Cells through Vesicles

1390 Incompletely Coated with Clathrin That Depend upon Actin for Internalization', PLoS

1391 Pathogens, 5(4). doi: 10.1371/journal.ppat.1000394. 
1392 Dawson, J. C., Legg, J. A. and Machesky, L. M. (2006) 'Bar domain proteins: a role

1393 in tubulation, scission and actin assembly in clathrin-mediated endocytosis', Trends

1394 in Cell Biology, 16(10), pp. 493-498. doi: 10.1016/j.tcb.2006.08.004.

1395 Day, P. M. et al. (2013) 'Identification of a role for the trans-Golgi network in human

1396 papillomavirus 16 pseudovirus infection.', Journal of virology, 87(7), pp. 3862-70.

1397 doi: 10.1128/JVI.03222-12.

1398 Day, P. M., Lowy, D. R. and Schiller, J. T. (2003) 'Papillomaviruses infect cells via a

1399 clathrin-dependent pathway', Virology, 307(1), pp. 1-11. doi: 10.1016/S0042-

1400 6822(02)00143-5.

1401 Derivery, E. et al. (2009) 'The Arp2/3 Activator WASH Controls the Fission

1402 of Endosomes through a Large Multiprotein Complex', Developmental Cell, 17(5), pp.

1403 712-723. doi: 10.1016/j.devcel.2009.09.010.

1404 DeTulleo, L. and Kirchhausen, T. (1998) 'The clathrin endocytic pathway in viral

1405 infection', EMBO Journal, 17(16), pp. 4585-4593. doi: 10.1093/emboj/17.16.4585.

1406 Doherty, G. J. and McMahon, H. T. (2009) 'Mechanisms of endocytosis.', Annual

1407 review of biochemistry, 78(April), pp. 857-902. doi:

1408 10.1146/annurev.biochem.78.081307.110540.

1409 Doorbar, J. (2005) 'The papillomavirus life cycle.', Journal of clinical virology $\square$ : the

1410 official publication of the Pan American Society for Clinical Virology, 32 Suppl

1411 1(SUPPL.), pp. S7-15. doi: 10.1016/j.jcv.2004.12.006.

1412 Duleh, S. N. and Welch, M. D. (2010) 'WASH and the Arp2/3 complex regulate

1413 endosome shape and trafficking', Cytoskeleton, 67(3), p. NA-NA. doi:

$1414 \quad 10.1002 / \mathrm{cm} .20437$.

1415 Dziduszko, A. and Ozbun, M. A. (2013) 'Annexin A2 and S100A10 regulate human

1416 papillomavirus type 16 entry and intracellular trafficking in human keratinocytes.',

1417 Journal of virology, 87(13), pp. 7502-15. doi: 10.1128/JVI.00519-13.

1418 Engel, S. et al. (2011) 'Role of endosomes in simian virus 40 entry and infection.',

1419 Journal of virology, 85(9), pp. 4198-4211. doi: 10.1128/JVI.02179-10.

1420 Evander, M. et al. (1997) 'Identification of the alpha6 integrin as a candidate receptor

1421 for papillomaviruses.', Journal of virology, 71(3), pp. 2449-56. Available at:

1422 http://www.ncbi.nlm.nih.gov/pubmed/9032382\%5Cnhttp://www.pubmedcentral.nih.go

1423 v/articlerender.fcgi?artid=PMC191355.

1424 Ezratty, E. J., Partridge, M. A. and Gundersen, G. G. (2005) 'Microtubule-induced

1425 focal adhesion disassembly is mediated by dynamin and focal adhesion kinase', 
1426 Nature Cell Biology, 7(6), pp. 581-590. doi: 10.1038/ncb1262.

1427 Fast, L. A. et al. (2018) 'Inhibition of tetraspanin functions impairs human

1428 papillomavirus and cytomegalovirus infections', International Journal of Molecular

1429 Sciences, 19(10). doi: 10.3390/ijms19103007.

1430 Florin, L. and Lang, T. (2018) 'Tetraspanin Assemblies in Virus Infection', Frontiers in

1431 Immunology, 9(May), pp. 1-9. doi: 10.3389/fimmu.2018.01140.

1432 Gallop, J. L. et al. (2006) 'Mechanism of endophilin N-BAR domain-mediated

1433 membrane curvature.', The EMBO journal, 25(12), pp. 2898-2910. doi:

1434 10.1038/sj.emboj.7601174.

1435 Giroglou, T. et al. (2001) 'Human Papillomavirus Infection Requires Cell Surface

1436 Heparan Sulfate', Journal of Virology, 75(3), pp. 1565-1570. doi:

1437 10.1128/JVI.75.3.1565.

1438 Gomez, T. S. and Billadeau, D. D. (2009) 'A FAM21-Containing WASH Complex

1439 Regulates Retromer-Dependent Sorting', Developmental Cell, 17(5), pp. 699-711.

1440 doi: 10.1016/j.devcel.2009.09.009.

1441 Hafezi, W. et al. (2012) 'Entry of herpes simplex virus type 1 (HSV-1) into the distal

1442 axons of trigeminal neurons favors the onset of nonproductive, silent infection', PLoS

1443 Pathogens, 8(5). doi: 10.1371/journal.ppat.1002679.

1444 Hänisch, J. et al. (2010) 'Molecular dissection of Salmonella-induced membrane

1445 ruffling versus invasion', Cellular Microbiology, 12(1), pp. 84-98. doi: 10.1111/j.1462-

1446 5822.2009.01380.x.

1447 Hao, Y. et al. (2013) 'Regulation of WASH-Dependent Actin Polymerization and

1448 Protein Trafficking by Ubiquitination', Cell, 152(5), pp. 1051-1064. doi:

1449 10.1016/j.cell.2013.01.051.

1450 Harbour, M. E., Breusegem, S. Y. and Seaman, M. N. J. (2012) 'Recruitment of the

1451 endosomal WASH complex is mediated by the extended "tail" of Fam21 binding to

1452 the retromer protein Vps35', Biochemical Journal, 442(1), pp. 209-220. doi:

1453 10.1042/BJ20111761.

1454 Hayer, A. et al. (2010) 'Biogenesis of caveolae: Stepwise assembly of large caveolin

1455 and cavin complexes', Traffic, 11(3), pp. 361-382. doi: 10.1111/j.1600-

1456 0854.2009.01023.x.

1457 Helenius, A. (2018) 'Virus Entry: Looking Back and Moving Forward', Journal of

1458 Molecular Biology. Elsevier Ltd, 430(13), pp. 1853-1862. doi:

$1459 \quad$ 10.1016/j.jmb.2018.03.034. 
1460 Helenius, A. (2020) 'Standing on the Shoulders of Viruses', Annual Review of

1461 Biochemistry, 89, pp. 21-43. doi: 10.1146/annurev-biochem-011320-103928.

1462 Helfer, E. et al. (2013) 'Endosomal recruitment of the WASH complex: Active

1463 sequences and mutations impairing interaction with the retromer', Biology of the Cell,

1464 105(5), pp. 191-207. doi: 10.1111/boc.201200038.

1465 Heuser, J. and Evans, L. (1980) 'Three-dimensional visualization of coated vesicle

1466 formation in fibroblasts', Journal of Cell Biology, 84(3), pp. 560-583. doi:

1467 10.1083/jcb.84.3.560.

1468 Hinshaw, J. E. and Schmid, S. L. (1995) 'Dynamin self-assembles into rings

1469 suggesting a mechanism for coated vesicle budding.', Nature, 374(6518), pp. 190-2.

1470 doi: 10.1038/374190a0.

1471 Huang, C.-Y. et al. (2008) 'A novel cellular protein, VPEF, facilitates vaccinia virus

1472 penetration into HeLa cells through fluid phase endocytosis.', Journal of virology,

1473 82(16), pp. 7988-99. doi: 10.1128/JVI.00894-08.

1474 Humbel, B. M., Stierhof, Y.-D. (2009) 'Cryosectioning according to Tokuyasu', in

1475 Cavalier, A., Spehner, D., Humbel, B. M. (ed.) Handbook of cryopreparation methods

1476 for electron microscopy.

1477 Hwang, S., Takimoto, T. and Hemler, M. E. (2019) 'Integrin-independent support of

1478 cancer drug resistance by tetraspanin CD151', Cellular and Molecular Life Sciences.

1479 Springer International Publishing, 76(8), pp. 1595-1604. doi: 10.1007/s00018-019-

1480 03014-7.

1481 Jao, C. C. et al. (2010) 'Roles of amphipathic helices and the bin/amphiphysin/rvs

1482 (BAR) domain of endophilin in membrane curvature generation', Journal of Biological

1483 Chemistry, 285(26), pp. 20164-20170. doi: 10.1074/jbc.M110.127811.

1484 Jia, D. et al. (2012) 'Multiple repeat elements within the FAM21 tail link the WASH

1485 actin regulatory complex to the retromer', Molecular Biology of the Cell, 23, pp.

1486 2352-2361. doi: 10.1091/mbc.E11-12-1059.

1487 Johannsdottir, H. K. et al. (2009) 'Host cell factors and functions involved in vesicular

1488 stomatitis virus entry.', Journal of virology, 83(1), pp. 440-53. doi:

1489 10.1128/JVI.01864-08.

1490 Johnson, K. M. et al. (2009) 'Role of Heparan Sulfate in Attachment to and Infection

1491 of the Murine Female Genital Tract by Human Papillomavirus', Journal of Virology,

1492 83(5), pp. 2067-2074. doi: 10.1128/jvi.02190-08.

1493 Jones, J. C. R., Hopkinson, S. B. and Goldfinger, L. E. (1998) 'Structure and 
1494 assembly of hemidesmosomes', BioEssays, 20(6), pp. 488-494. doi:

1495 10.1002/(SICI)1521-1878(199806)20:6<488::AID-BIES7>3.0.CO;2-I.

1496 Kage, F. et al. (2017) ‘FMNL formins boost lamellipodial force generation', Nature

1497 Communications, 8. doi: 10.1038/ncomms14832.

1498 Kaksonen, M. and Roux, A. (2018) 'Mechanisms of clathrin-mediated endocytosis',

1499 Nature Reviews Molecular Cell Biology. Nature Publishing Group, 19(5), pp. 313-

1500 326. doi: 10.1038/nrm.2017.132.

1501 Kamentsky, L. et al. (2011) 'Improved structure, function and compatibility for

1502 cellprofiler: Modular high-throughput image analysis software', Bioinformatics, 27(8),

1503 pp. 1179-1180. doi: 10.1093/bioinformatics/btr095.

1504 Kirkham, M. et al. (2005) 'Ultrastructural identification of uncoated caveolin-

1505 independent early endocytic vehicles', Journal of Cell Biology, 168(3), pp. 465-476.

1506 doi: 10.1083/jcb.200407078.

1507 Kvainickas, A. et al. (2017) 'Cargo-selective SNX-BAR proteins mediate retromer

1508 trimer independent retrograde transport.', The Journal of cell biology, p.

1509 jcb.201702137. doi: 10.1083/jcb.201702137.

1510 Lakshminarayan, R. et al. (2014) 'Galectin-3 drives glycosphingolipid-dependent

1511 biogenesis of clathrin-independent carriers', Nature Cell Biology, 16(6), pp. 592-603.

1512 doi: 10.1038/ncb2970.

1513 Landry, J. J. M. et al. (2013) 'The genomic and transcriptomic landscape of a HeLa

1514 cell line.', G3 (Bethesda, Md.), 3(8), pp. 1213-24. doi: 10.1534/g3.113.005777.

1515 Liberali, P. et al. (2008) 'The closure of Pak1-dependent macropinosomes requires

1516 the phosphorylation of CtBP1/BARS.', The EMBO journal, 27(7), pp. 970-81. doi:

1517 10.1038/emboj.2008.59.

1518 Lim, J. P. and Gleeson, P. A. (2011) 'Macropinocytosis: an endocytic pathway for

1519 internalising large gulps', Immunology and Cell Biology, 89(8), pp. 836-843. doi:

1520 10.1038/icb.2011.20.

1521 Linardopoulou, E. V et al. (2007) 'Human subtelomeric WASH genes encode a new

1522 subclass of the WASP family.', PLoS genetics, 3(12), p. e237. doi:

1523 10.1371/journal.pgen.0030237.

1524 Lipovsky, A. et al. (2013) 'Genome-wide siRNA screen identifies the retromer as a

1525 cellular entry factor for human papillomavirus.', Proceedings of the National Academy

1526 of Sciences of the United States of America, 110(18), pp. 7452-7. doi:

1527 10.1073/pnas.1302164110. 
1528 Lipovsky, A. et al. (2015) 'Application of the proximity-dependent assay and

1529 fluorescence imaging approaches to study viral entry pathways.', Methods in

1530 molecular biology (Clifton, N.J.), 1270, pp. 437-51. doi: 10.1007/978-1-4939-2309-

1531 0_30.

1532 Liu, L. et al. (2007) 'Tetraspanin CD151 promotes cell migration by regulating integrin

1533 trafficking', Journal of Biological Chemistry, 282(43), pp. 31631-31642. doi:

1534 10.1074/jbc.M701165200.

1535 Loerke, D. et al. (2009) 'Cargo and Dynamin Regulate Clathrin-Coated Pit

1536 Maturation', PLoS Biology, 7(3). doi: 10.1371/journal.pbio.1000057.

1537 Machesky, L. M. and Insall, R. H. (1998) 'Scar1 and the related Wiskott-Aldrich

1538 syndrome protein, WASP, regulate the actin cytoskeleton through the Arp2/3

1539 complex', Current Biology, 8(25), pp. 1347-1356. doi: 10.1016/S0960-

1540 9822(98)00015-3.

1541 Marsh, M. and Helenius, A. (1980) 'Adsorptive endocytosis of Semliki Forest virus',

1542 Journal of Molecular Biology, 142(3), pp. 439-454. doi: 10.1016/0022-

1543 2836(80)90281-8.

1544 Marsh, M. and Helenius, A. (2006) 'Virus entry: Open sesame', Cell, 124(4), pp. 729_

1545 740. doi: 10.1016/j.cell.2006.02.007.

1546 Marsh, M., Kielian, M. C. and Helenius, A. (1984) 'Semliki forest virus entry and the

1547 endocytic pathway', Biochemical Society Transactions, 12(6), pp. 981-983. doi:

$1548 \quad 10.1042 /$ bst0120981.

1549 Mazzon, M. and Mercer, J. (2014) 'Lipid Interactions During Virus Entry and

1550 Infection', Cellular Microbiology, 16(September), p. n/a-n/a. doi: 10.1111/cmi.12340.

1551 McMahon, H. T. and Boucrot, E. (2011) 'Molecular mechanism and physiological

1552 functions of clathrin-mediated endocytosis', Nature Publishing Group, 12(8), pp. 517-

1553 533. doi: 10.1038/nrm3151.

1554 Ménager, M. M. and Littman, D. R. (2016) 'Actin Dynamics Regulates Dendritic Cell-

1555 Mediated Transfer of HIV-1 to T Cells', Cell, 164(4), pp. 695-709. doi:

1556 10.1016/j.cell.2015.12.036.

1557 Mercer, J. and Helenius, A. (2008) 'Vaccinia virus uses macropinocytosis and

1558 apoptotic mimicry to enter host cells.', Science (New York, N.Y.), 320(5875), pp.

1559 531-535. doi: 10.1126/science.1155164.

1560 Mercer, J., Schelhaas, M. and Helenius, A. (2010) 'Virus entry by endocytosis.',

1561 Annual review of biochemistry, 79, pp. 803-33. doi: 10.1146/annurev-biochem- 
$1562060208-104626$.

1563 Merrifield, C. J. et al. (2002) 'Imaging actin and dynamin recruitment during

1564 invagination of single clathrin-coated pits', Nat Cell Biol, 4(9), pp. 691-698. doi:

$156510.1038 /$ ncb837.

1566 Merrifield, C. J. (2004) 'Seeing is believing: Imaging actin dynamics at single sites of

1567 endocytosis', Trends in Cell Biology, 14(7), pp. 352-358. doi:

1568 10.1016/j.tcb.2004.05.008.

1569 Merrifield, C. J. and Kaksonen, M. (2014) 'Endocytic accessory factors and regulation

1570 of clathrin-mediated endocytosis.', Cold Spring Harbor perspectives in biology, 6(11),

1571 p. a016733. doi: 10.1101/cshperspect.a016733.

1572 Mikuličić, S. et al. (2019) 'ADAM17-dependent signaling is required for oncogenic

1573 human papillomavirus entry platform assembly.', eLife, 8. doi: 10.7554/eLife.44345.

1574 Mirabello, L. et al. (2017) 'HPV16 E7 Genetic Conservation Is Critical to

1575 Carcinogenesis', Cell, 170(6), pp. 1164-1174.e6. doi: 10.1016/j.cell.2017.08.001.

1576 Mooren, O. L., Galletta, B. J. and Cooper, J. A. (2012) 'Roles for Actin Assembly in

1577 Endocytosis', Annu. Rev. Biochem, 81(May), pp. 661-86. doi: 10.1146/annurev-

1578 biochem-060910-094416.

1579 Morlot, S. and Roux, A. (2013) 'Mechanics of dynamin-mediated membrane fission.',

1580 Annual review of biophysics, 42, pp. 629-49. doi: 10.1146/annurev-biophys-050511-

$1581 \quad 102247$.

1582 Nicola, A. V. et al. (2005) 'Herpes Simplex Virus Type 1 Enters Human Epidermal

1583 Keratinocytes, but Not Neurons, via a pH-Dependent Endocytic Pathway', Journal of

1584 Virology, 79(12), pp. 7609-7616. doi: 10.1128/jvi.79.12.7609-7616.2005.

1585 Owaribe, K. et al. (1990) 'The hemidesmosomal plaque', Differentiation, 45(3), pp.

1586 207-220. doi: 10.1111/j.1432-0436.1990.tb00475.x.

1587 Pelkmans, L. et al. (2004) 'Caveolin-stabilized membrane domains as multifunctional

1588 transport and sorting devices in endocytic membrane traffic', Cell, 118(6), pp. 767-

1589 780. doi: 10.1016/j.cell.2004.09.003.

1590 Pelkmans, L., Püntener, D. and Helenius (2002) 'Local Actin Polymerization and

1591 Dynamin Recruitment in SV40-Induced Internalization of Caveolae', Science,

1592 296(5567), pp. 535-539. doi: 10.1126/science.1069784.

1593 Popa, A. et al. (2015) 'Direct Binding of Retromer to Human Papillomavirus Type 16

1594 Minor Capsid Protein L2 Mediates Endosome Exit during Viral Infection', PLoS

1595 Pathogens, 11(2), pp. 1-21. doi: 10.1371/journal.ppat.1004699. 
1596 Popoff, V. et al. (2007) 'The retromer complex and clathrin define an early endosomal

1597 retrograde exit site', Journal of Cell Science, 120(12), pp. 2022-2031. doi:

1598 10.1242/jcs.003020.

1599 Pyeon, D. et al. (2009) 'Establishment of human papillomavirus infection requires cell

1600 cycle progression', PLoS Pathogens, 5(2). doi: 10.1371/journal.ppat.1000318.

1601 Qualmann, B. and Kelly, R. B. (2000) 'Syndapin isoforms participate in receptor-

1602 mediated endocytosis and actin organization', Journal of Cell Biology, 148(5), pp.

1603 1047-1061. doi: 10.1083/jcb.148.5.1047.

1604 Qualmann, B., Koch, D. and Kessels, M. M. (2011) 'Let's go bananas: revisiting the

1605 endocytic BAR code.', The EMBO journal, 30(17), pp. 3501-15. doi:

1606 10.1038/emboj.2011.266.

1607 Quirin, K. et al. (2008) 'Lymphocytic choriomeningitis virus uses a novel endocytic

1608 pathway for infectious entry via late endosomes', Virology, 378(1), pp. 21-33. doi:

1609 10.1016/j.virol.2008.04.046.

1610 Raff, A. B. et al. (2013) 'The Evolving Field of Human Papillomavirus Receptor

1611 Research: a Review of Binding and Entry', Journal of Virology, 87(11), pp. 6062-

1612 6072. doi: 10.1128/JVI.00330-13.

1613 Ran, F. A. et al. (2013) 'Genome engineering using the CRISPR-Cas9 system.', Nat

1614 Protoc, 8(11), pp. 2281-2308. doi: 10.1038/nprot.2013.143.

1615 Richards, R. M. et al. (2006) 'Cleavage of the papillomavirus minor capsid protein,

1616 L2, at a furin consensus site is necessary for infection.', Proceedings of the National

1617 Academy of Sciences of the United States of America, 103(5), pp. 1522-7. doi:

1618 10.1073/pnas.0508815103.

1619 Riedl, J. et al. (2008) 'Lifeact: a versatile marker to visualize F-actin.', Nature

1620 methods, 5(7), pp. 605-7. doi: 10.1038/nmeth.1220.

1621 Rizvi, S. A. et al. (2009) 'Identification and Characterization of a Small Molecule

1622 Inhibitor of Formin-Mediated Actin Assembly', Chemistry and Biology. Elsevier Ltd,

1623 16(11), pp. 1158-1168. doi: 10.1016/j.chembiol.2009.10.006.

1624 Roberts, J. N. et al. (2007) 'Genital transmission of HPV in a mouse model is

1625 potentiated by nonoxynol-9 and inhibited by carrageenan', Nature Medicine, 13(7),

1626 pp. 857-861. doi: 10.1038/nm1598.

1627 Rojek, J. M., Perez, M. and Kunz, S. (2008) 'Cellular Entry of Lymphocytic

1628 Choriomeningitis Virus', Journal of Virology, 82(3), pp. 1505-1517. doi:

$1629 \quad 10.1128 /$ jvi.01331-07. 
1630 Rottner, K., Hänisch, J. and Campellone, K. G. (2010) 'WASH, WHAMM and JMY:

1631 regulation of Arp2/3 complex and beyond.', Trends in cell biology, 20(11), pp. 650-

1632 61. doi: 10.1016/j.tcb.2010.08.014.

1633 Rust, M. J. et al. (2004) 'Assembly of endocytic machinery around individual

1634 influenza viruses during viral entry', Nature Structural and Molecular Biology, 11(6),

1635 pp. 567-573. doi: 10.1038/nsmb769.

1636 Saalfeld, S. and Tomancák, P. (2008) 'Automatic landmark correspondence

1637 detection for ImageJ', Proceedings of the ImageJ User and Developer Conference,

1638 pp. 128-133. Available at: http://fly.mpi-

1639 cbg.de/ saalfeld/Publications/download/imagejconf2008.pdf.

1640 de Sanjose, S. et al. (2010) 'Human papillomavirus genotype attribution in invasive

1641 cervical cancer: a retrospective cross-sectional worldwide study', The Lancet

1642 Oncology, 11(11), pp. 1048-1056. doi: 10.1016/S1470-2045(10)70230-8.

1643 Scheffer, K. D. et al. (2013) 'Tetraspanin CD151 Mediates Papillomavirus Type 16

1644 Endocytosis', Journal of Virology, 87(6), pp. 3435-3446. doi: 10.1128/JVI.02906-12.

1645 Schelhaas, M. et al. (2003) 'Herpes simplex virus type 1 exhibits a tropism for basal

1646 entry in polarized epithelial cells', Journal of General Virology, 84(9), pp. 2473-2484.

1647 doi: 10.1099/vir.0.19226-0.

1648 Schelhaas, M. et al. (2008) 'Human papillomavirus type 16 entry: Retrograde cell

1649 surface transport along actin-rich protrusions', PLoS Pathogens, 4(9). doi:

$1650 \quad 10.1371 /$ journal.ppat.1000148.

1651 Schelhaas, M. et al. (2012) 'Entry of human papillomavirus type 16 by actin-

1652 dependent, clathrin- and lipid raft-independent endocytosis.', PLoS pathogens, 8(4),

1653 p. e1002657. doi: 10.1371/journal.ppat.1002657.

1654 Schindelin, J. et al. (2012) 'Fiji: An open-source platform for biological-image

1655 analysis', Nature Methods, 9(7), pp. 676-682. doi: 10.1038/nmeth.2019.

1656 Schmid, S. L. and Conner, S. D. (2003) 'Regulated portals of entry into the cell',

1657 Nature, 422(March), pp. 37-44. Available at: www.nature.com/nature.

1658 Seaman, M. N. J. (2012) 'The retromer complex - endosomal protein recycling and

1659 beyond.', Journal of cell science, 125(Pt 20), pp. 4693-702. doi: 10.1242/jcs.103440.

1660 Selinka, H.-C. et al. (2007) 'Inhibition of transfer to secondary receptors by heparan

1661 sulfate-binding drug or antibody induces noninfectious uptake of human

1662 papillomavirus.', Journal of virology, 81(20), pp. 10970-80. doi: 10.1128/JVI.00998-

166307. 
1664 Shikama, N. et al. (1999) 'A novel cofactor for p300 that regulates the p53 response',

1665 Molecular Cell, 4(3), pp. 365-376. doi: 10.1016/S1097-2765(00)80338-X.

1666 Shin, N. et al. (2008) 'SNX9 regulates tubular invagination of the plasma membrane

1667 through interaction with actiAn cytoskeleton and dynamin 2.', Journal of cell science,

1668 121(Pt 8), pp. 1252-63. doi: 10.1242/jcs.016709.

1669 Siddiqa, A. et al. (2018) 'Human Papillomavirus 16 Infection Induces VAP-Dependent

1670 Endosomal Tubulation', Journal of Virology. Edited by R. M. Longnecker, 92(6), pp.

1671 1-14. doi: 10.1128/JVI.01514-17.

1672 Sieczkarski, S. B. and Whittaker, G. R. (2002) 'Influenza virus can enter and infect

1673 cells in the absence of clathrin-mediated endocytosis.', Journal of virology, 76(20),

1674 pp. 10455-64. doi: 10.1128/jvi.76.20.10455-10464.2002.

1675 Simonetti, B. et al. (2017) 'Sequence-dependent cargo recognition by SNX-BARs

1676 mediates retromer-independent transport of CI-MPR', Journal of Cell Biology,

1677 216(11), pp. 3695-3712. doi: 10.1083/jcb.201703015.

1678 Singh, I. and Helenius, A. (1992) 'Role of ribosomes in Semliki Forest virus

1679 nucleocapsid uncoating.', Journal of Virology, 66(12), pp. 7049-7058. doi:

$1680 \quad 10.1128 /$ jvi.66.12.7049-7058.1992.

1681 Smith, J. L. et al. (2008) 'Caveolin-1-Dependent Infectious Entry of Human

1682 Papillomavirus Type 31 in Human Keratinocytes Proceeds to the Endosomal

1683 Pathway for pH-Dependent Uncoating', Journal of Virology, 82(19), pp. 9505-9512.

1684 doi: 10.1128/jvi.01014-08.

1685 Snijder, B. et al. (2012) 'Single-cell analysis of population context advances RNAi

1686 screening at multiple levels.', Molecular systems biology, 8(579), p. 579. doi:

$1687 \quad 10.1038 / \mathrm{msb} .2012 .9$.

1688 Sochacki, K. A. et al. (2014) 'Correlative super-resolution fluorescence and metal-

1689 replica transmission electron microscopy', Nature Methods, 11(3), pp. 305-308. doi:

$169010.1038 /$ nmeth.2816.

1691 Söderberg, O. et al. (2006) 'Direct observation of individual endogenous protein

1692 complexes in situ by proximity ligation', Nature Methods, 3(12), pp. 995-1000. doi:

$169310.1038 /$ nmeth947.

1694 Sokac, A. M. et al. (2003) 'Cdc42-dependent actin polymerization during

1695 compensatory endocytosis in Xenopus eggs.', Nature cell biology, 5(8), pp. 727-732.

1696 doi: 10.1038/ncb1025.

1697 Soulet, F. et al. (2005) 'SNX9 Regulates Dynamin Assembly and Is Required for 
1698 Efficient Clathrin-mediated Endocytosis', Molecular Biology of the Cell, 16(4), pp.

1699 2058-2067. doi: 10.1091/mbc.e04-11-1016.

1700 Spoden, G. et al. (2008) 'Clathrin- and caveolin-independent entry of human

1701 papillomavirus type 16--involvement of tetraspanin-enriched microdomains (TEMs).',

1702 PloS one, 3(10), p. e3313. doi: 10.1371/journal.pone.0003313.

1703 Stechmann, B. et al. (2010) 'Inhibition of retrograde transport protects mice from

1704 lethal ricin challenge', Cell, 141(2), pp. 231-242. doi: 10.1016/j.cell.2010.01.043.

1705 Steffen, A. et al. (2006) 'Filopodia Formation in the Absence of Functional WAVE-

1706 and Arp2/3-Complexes', Molecular Biology of the Cell. Edited by M. Ginsberg, 17(6),

1707 pp. 2581-2591. doi: 10.1091/mbc.e05-11-1088.

1708 Stoeber, M. et al. (2016) 'Model for the architecture of caveolae based on a flexible,

1709 net-like assembly of Cavin1 and Caveolin discs', Proceedings of the National

1710 Academy of Sciences of the United States of America, 113(50), pp. E8069-E8078.

1711 doi: 10.1073/pnas.1616838113.

1712 Stradal, T. E. and Scita, G. (2006) 'Protein complexes regulating Arp2/3-mediated

1713 actin assembly', Current Opinion in Cell Biology, 18(1), pp. 4-10. doi:

1714 10.1016/j.ceb.2005.12.003.

1715 Suetsugu, S. et al. (2003) 'Differential roles of WAVE1 and WAVE2 in dorsal and

1716 peripheral ruffle formation for fibroblast cell migration', Developmental Cell, 5(4), pp.

1717 595-609. doi: 10.1016/S1534-5807(03)00297-1.

1718 Surviladze, Z., Dziduszko, A. and Ozbun, M. A. (2012) 'Essential roles for soluble

1719 virion-associated heparan sulfonated proteoglycans and growth factors in human

1720 papillomavirus infections.', PLoS pathogens, 8(2), p. e1002519. doi:

1721 10.1371/journal.ppat.1002519.

1722 Sweitzer, S. M. and Hinshaw, J. E. (1998) 'Dynamin undergoes a GTP-dependent

1723 conformational change causing vesiculation', Cell, 93(6), pp. 1021-1029. doi:

1724 10.1016/S0092-8674(00)81207-6.

1725 Takei, K. et al. (1995) 'Tubular membrane invaginations coated by dynamin rings are

1726 induced by GTP-yS in nerve terminals', Nature, 374(6518), pp. 186-190. doi:

1727 10.1038/374186a0.

1728 Taylor, M. J., Lampe, M. and Merrifield, C. J. (2012) 'A feedback loop between

1729 dynamin and actin recruitment during clathrin-mediated endocytosis', PLoS Biology,

1730 10(4). doi: 10.1371/journal.pbio.1001302.

1731 Taylor, M. J., Perrais, D. and Merrifield, C. J. (2011) 'A high precision survey of the 
1732 molecular dynamics of mammalian clathrin-mediated endocytosis', PLoS Biology,

1733 9(3). doi: 10.1371/journal.pbio.1000604.

1734 Tokuyasu, K. T. (1980) 'Immunochemistry on ultrathin frozen sections', The

1735 Histochemical Journal, 12(4), pp. 381-403. doi: 10.1007/BF01011956.

1736 Tsarouhas, V. et al. (2019) 'WASH phosphorylation balances endosomal versus

1737 cortical actin network integrities during epithelial morphogenesis', Nature

1738 Communications. Springer US, 10(1). doi: 10.1038/s41467-019-10229-6.

1739 Valente, C., Luini, A. and Corda, D. (2013) 'Components of the CtBP1/BARS-

1740 dependent fission machinery', Histochemistry and Cell Biology, 140(4), pp. 407-421.

1741 doi: 10.1007/s00418-013-1138-1.

1742 Ventayol, P. S. and Schelhaas, M. (2015) 'Fluorescently labeled human

1743 papillomavirus pseudovirions for use in virus entry experiments', Current Protocols in

1744 Microbiology, 2015(May), pp. 14B.4.1-14B.4.22. doi:

1745 10.1002/9780471729259.mc14b04s37.

1746 de Vries, E. et al. (2011) 'Dissection of the influenza a virus endocytic routes reveals

1747 macropinocytosis as an alternative entry pathway', PLoS Pathogens, 7(3), p.

1748 e1001329. doi: 10.1371/journal.ppat.1001329.

1749 Walko, G., Castañón, M. J. and Wiche, G. (2015) 'Molecular architecture and

1750 function of the hemidesmosome', Cell and Tissue Research, 360(3), pp. 529-544.

1751 doi: 10.1007/s00441-015-2216-6.

1752 Webb, D. J., Parsons, J. T. and Horwitz, A. F. (2002) 'Adhesion assembly,

1753 disassembly and turnover in migrating cells - Over and over and over again', Nature

1754 Cell Biology, 4(4). doi: 10.1038/ncb0402-e97.

1755 Woodham, A. W. et al. (2012) 'The S100A10 subunit of the annexin A2

1756 heterotetramer facilitates L2-mediated human papillomavirus infection.', PloS one,

1757 7(8), p. e43519. doi: 10.1371/journal.pone.0043519.

1758 Xie, J. et al. (2020) 'TBC1D5-Catalyzed Cycling of Rab7 Is Required for Retromer-

1759 Mediated Human Papillomavirus Trafficking during Virus Entry', Cell Reports, 31(10).

1760 doi: 10.1016/j.celrep.2020.107750.

1761 Yarar, D., Waterman-Storer, C. M. and Schmid, S. L. (2007) 'SNX9 Couples Actin

1762 Assembly to Phosphoinositide Signals and Is Required for Membrane Remodeling

1763 during Endocytosis', Developmental Cell, 13(1), pp. 43-56. doi:

1764 10.1016/j.devcel.2007.04.014.

1765 Yong, X. et al. (2020) 'Mechanism of cargo recognition by retromerlinked SNX-BAR 
1766 proteins', PLoS Biology, 18(3), pp. 1-29. doi: 10.1371/journal.pbio.3000631.

1767 Yoon, C.-S. et al. (2001) 'a6 Integrin Is the Main Receptor of Human Papillomavirus

1768 Type 16 VLP', Biochemical and Biophysical Research Communications, 283(3), pp.

1769 668-673. doi: 10.1006/bbrc.2001.4838.

1770 Zhang, P. et al. (2018) 'Cell-Penetrating Peptide Mediates Intracellular Membrane

1771 Passage of Human Papillomavirus L2 Protein to Trigger Retrograde Trafficking', Cell,

1772 174(6), pp. 1465-1476.e13. doi: 10.1016/j.cell.2018.07.031.

1773 Zhong, Q. et al. (2002) 'Endosomal localization and function of sorting nexin 1.',

1774 Proceedings of the National Academy of Sciences of the United States of America,

1775 99(10), pp. 6767-6772. doi: 10.1073/pnas.092142699.

1776 Zuchero, J. B. et al. (2009) 'p53-cofactor JMY is a multifunctional actin nucleation

1777 factor', Nature Cell Biology, 11(4), pp. 451-459. doi: 10.1038/ncb1852.

1778 
A early stage

intermediate stage

late stage

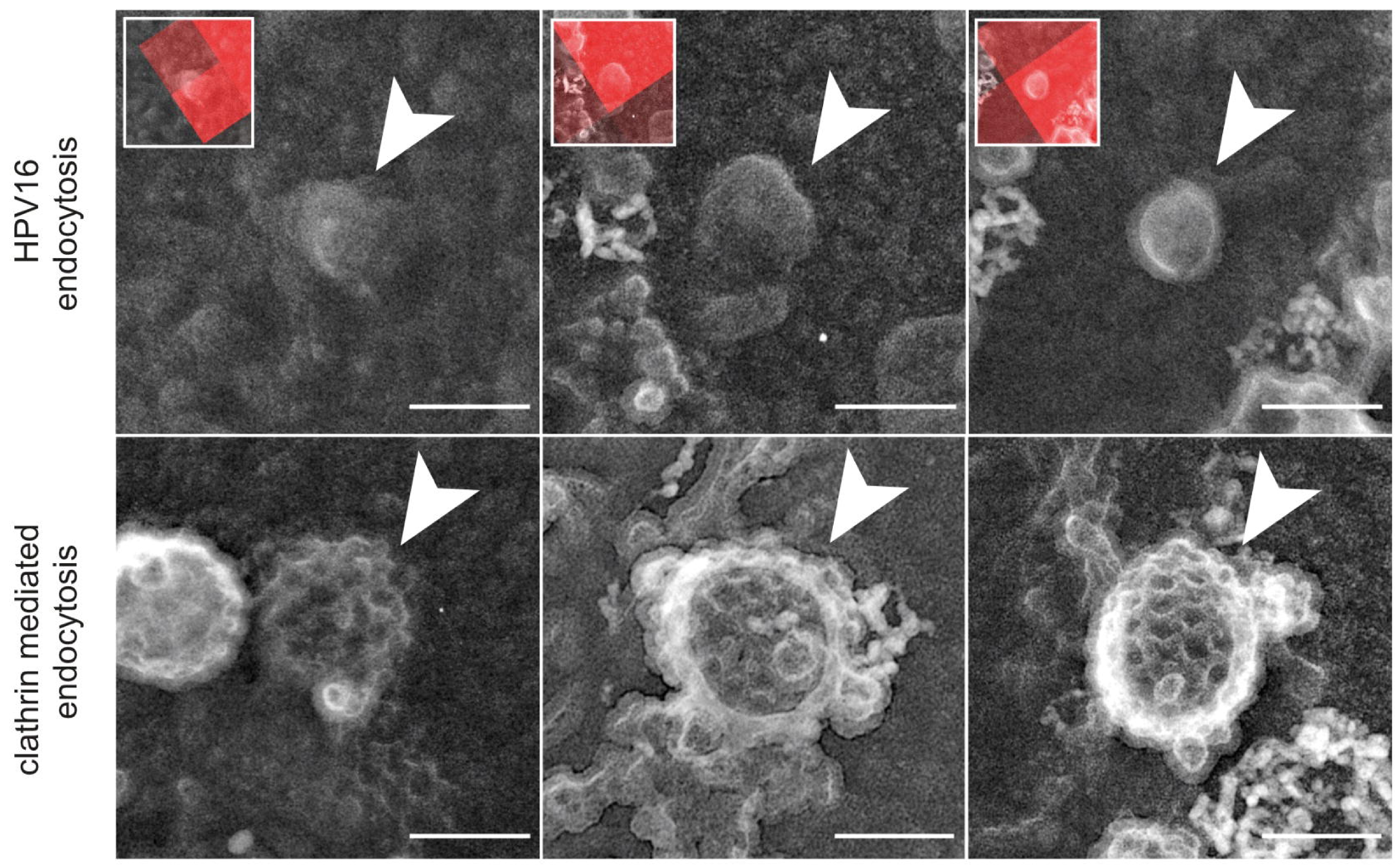

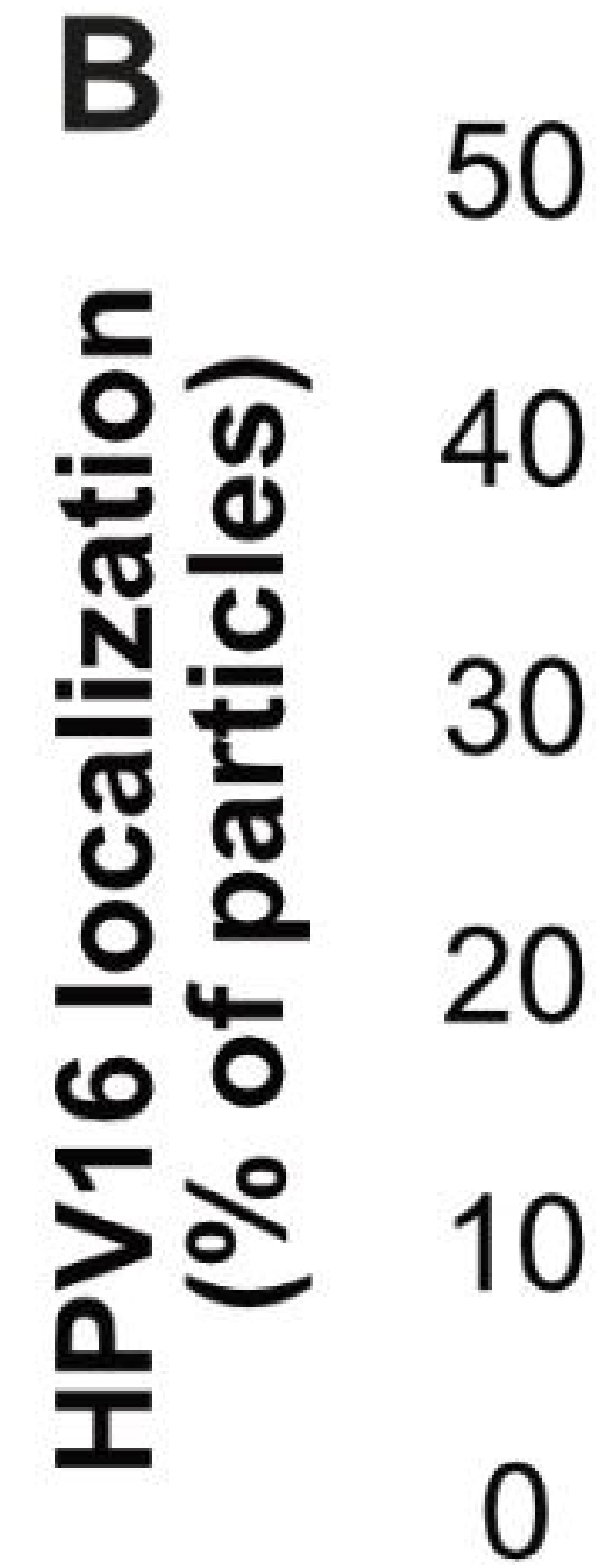

DMSO a cytoD

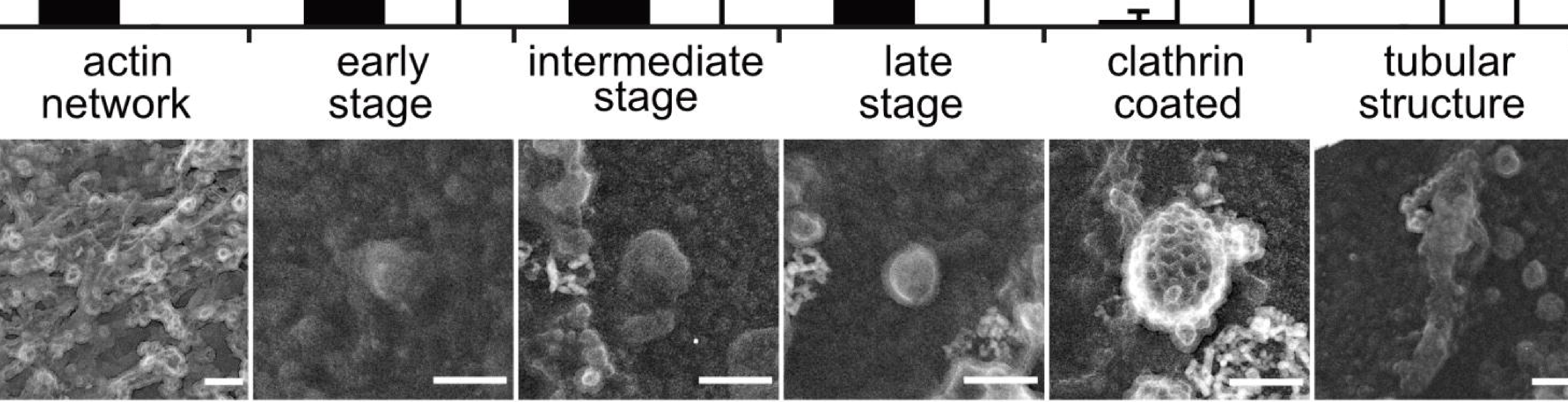

c

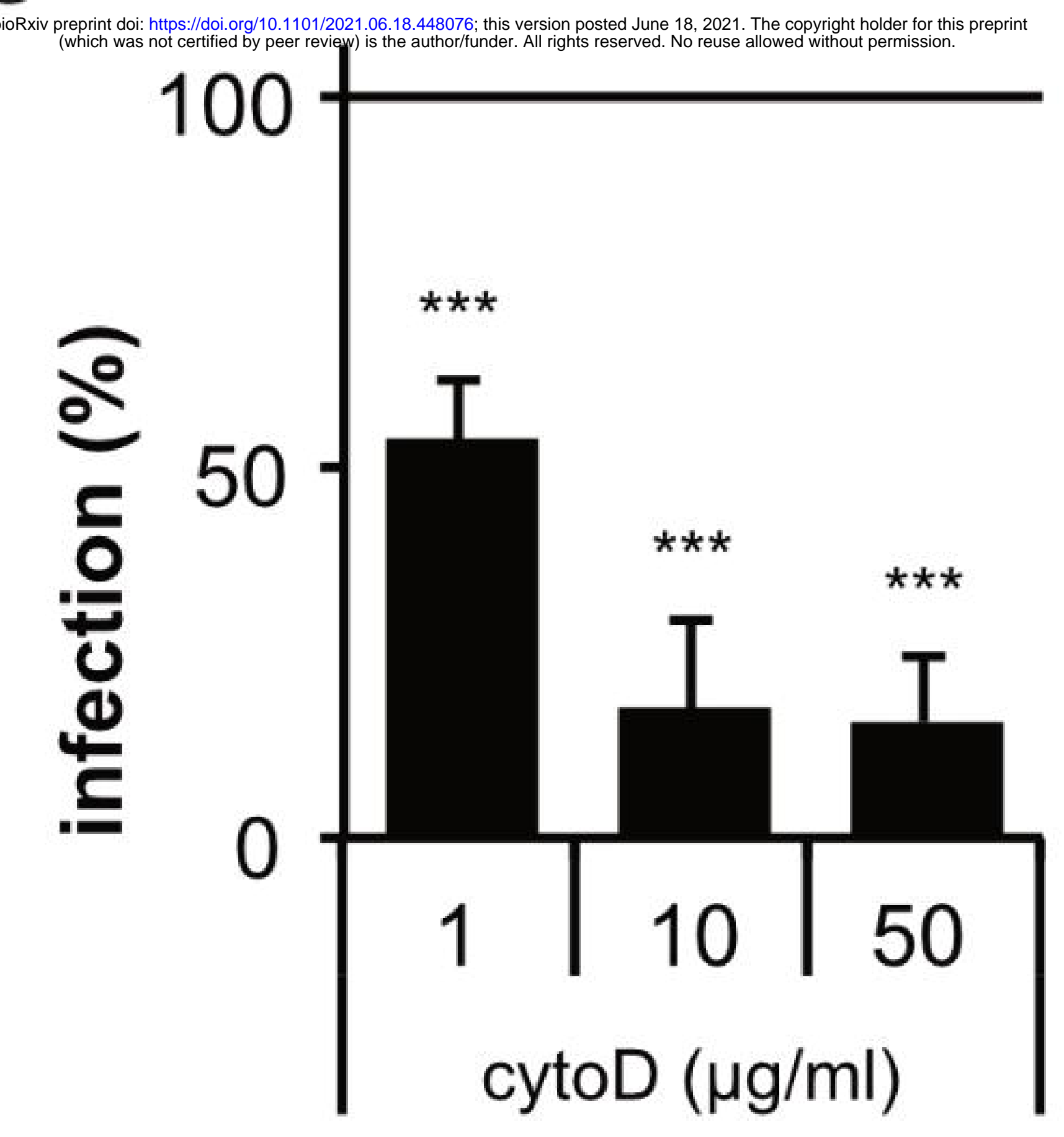

D

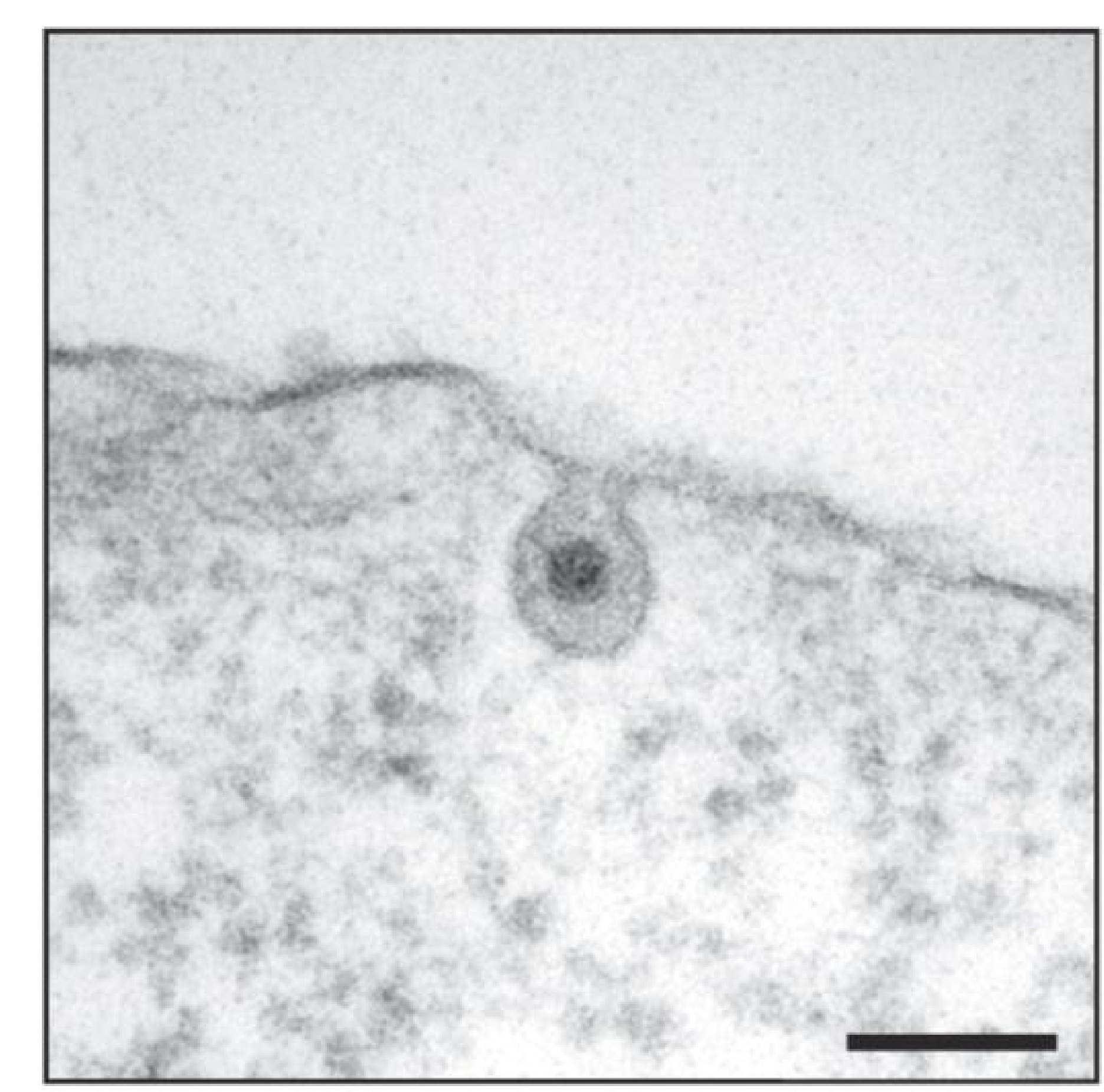

cytoD

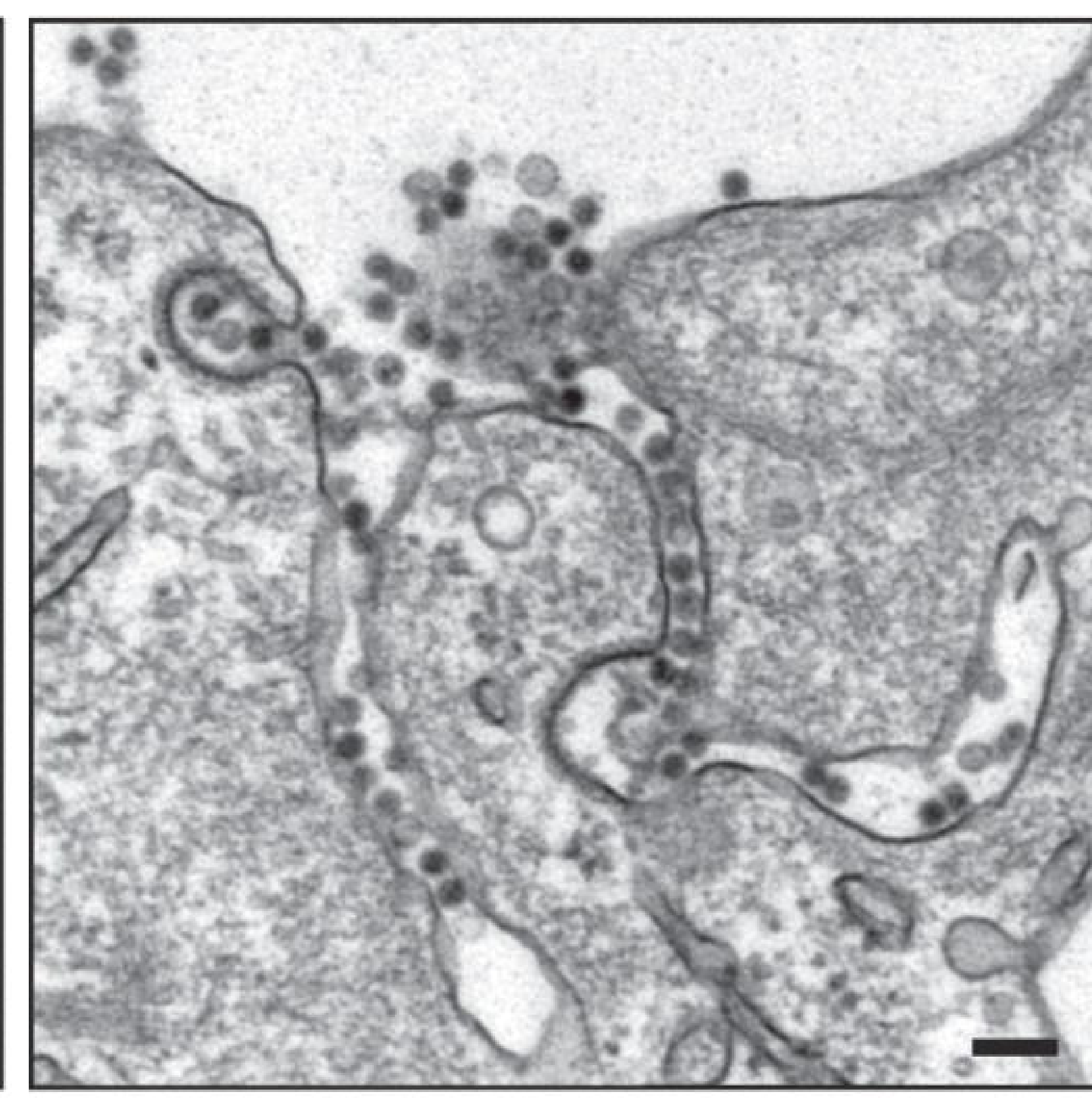



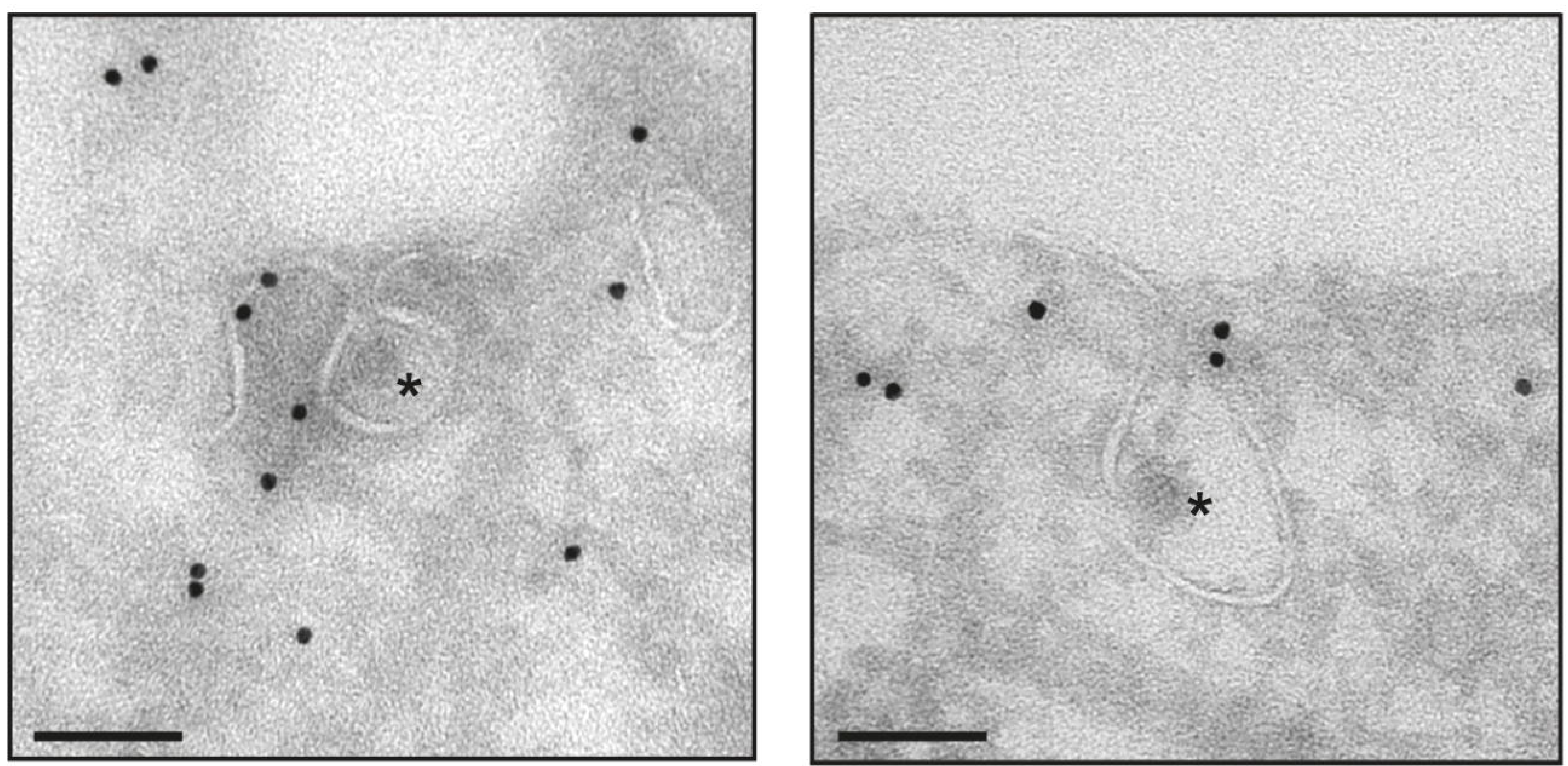

B
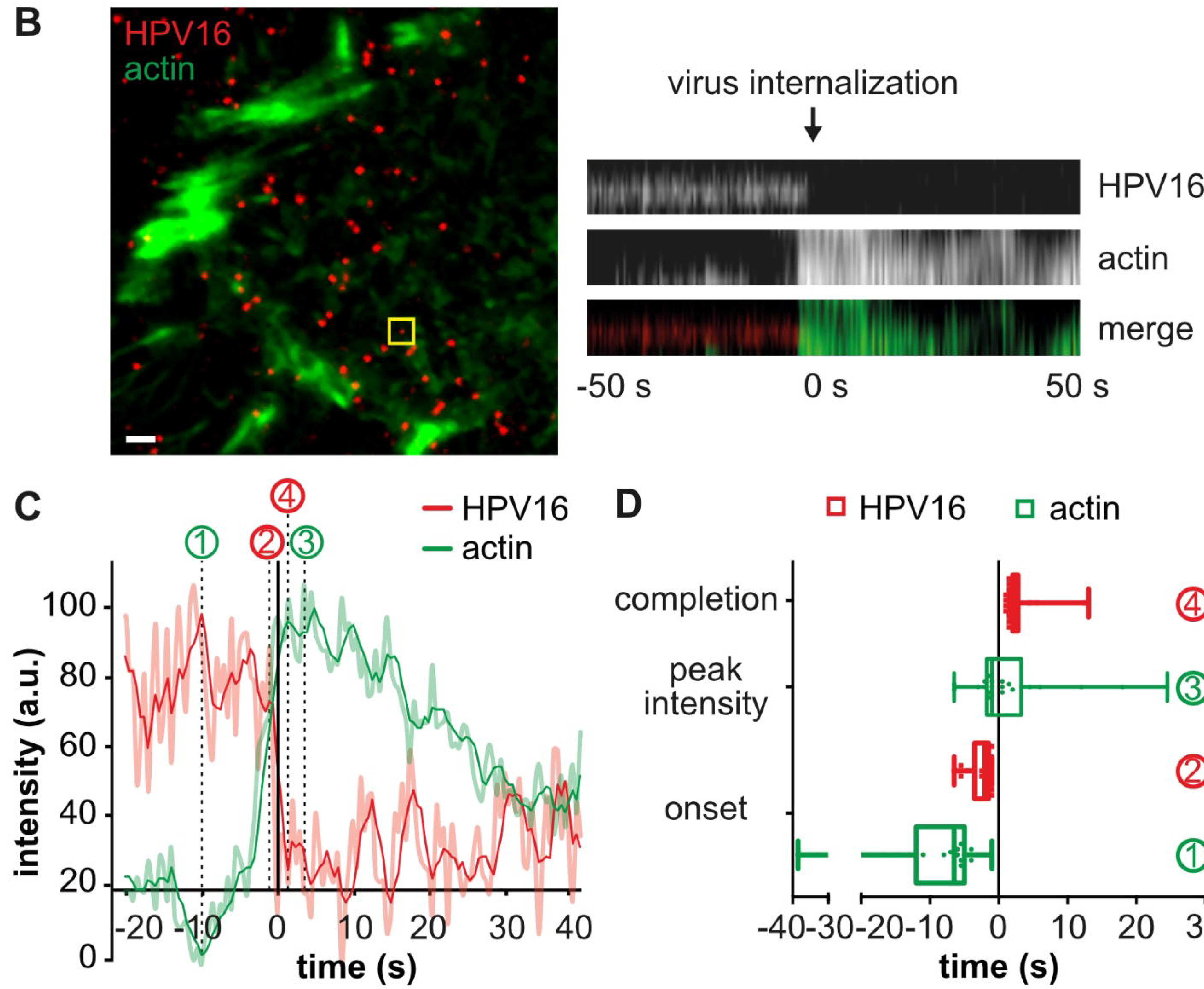

D $\quad$ HPV16 a actin

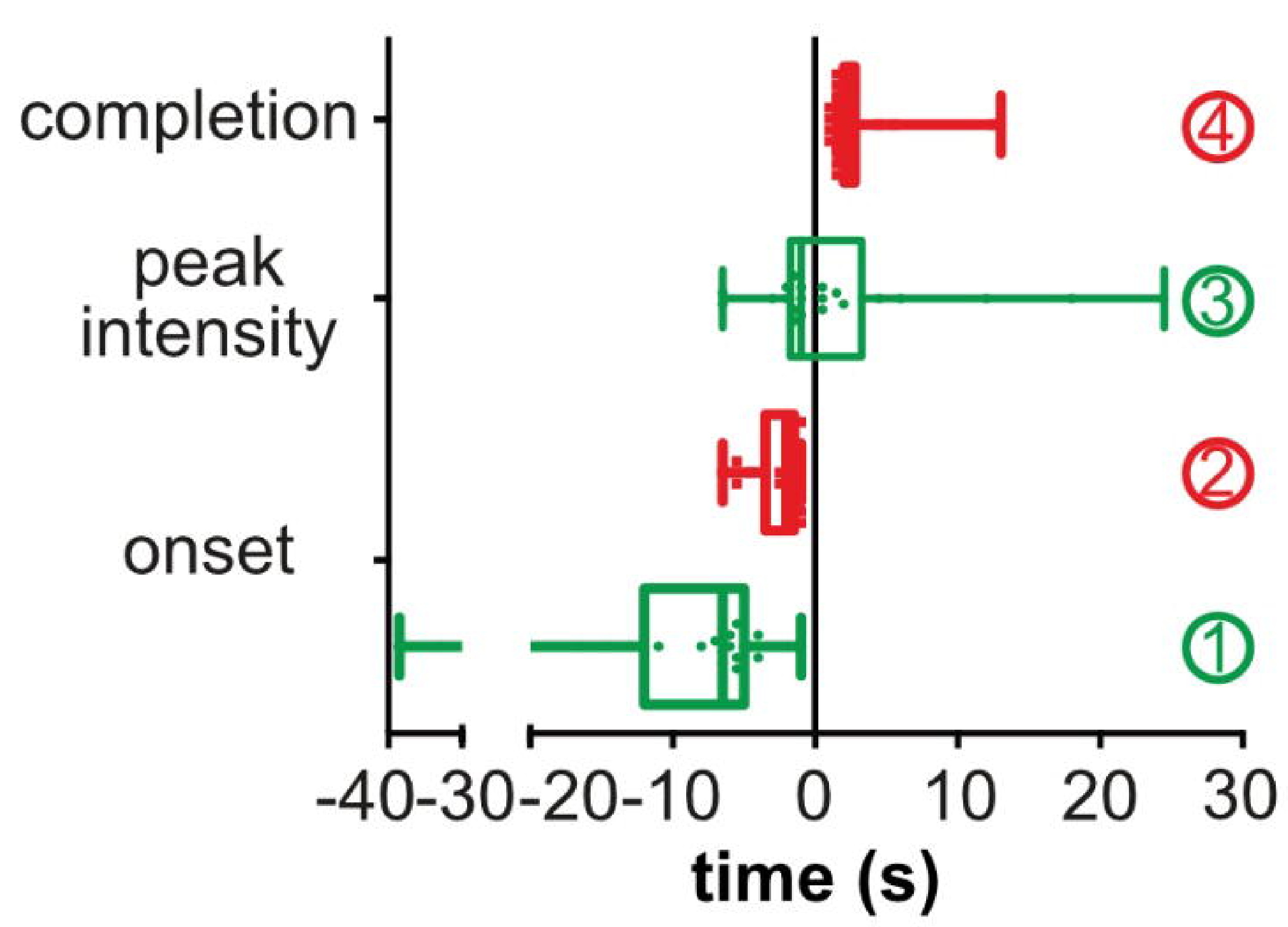


A

घHPV16 DVSV
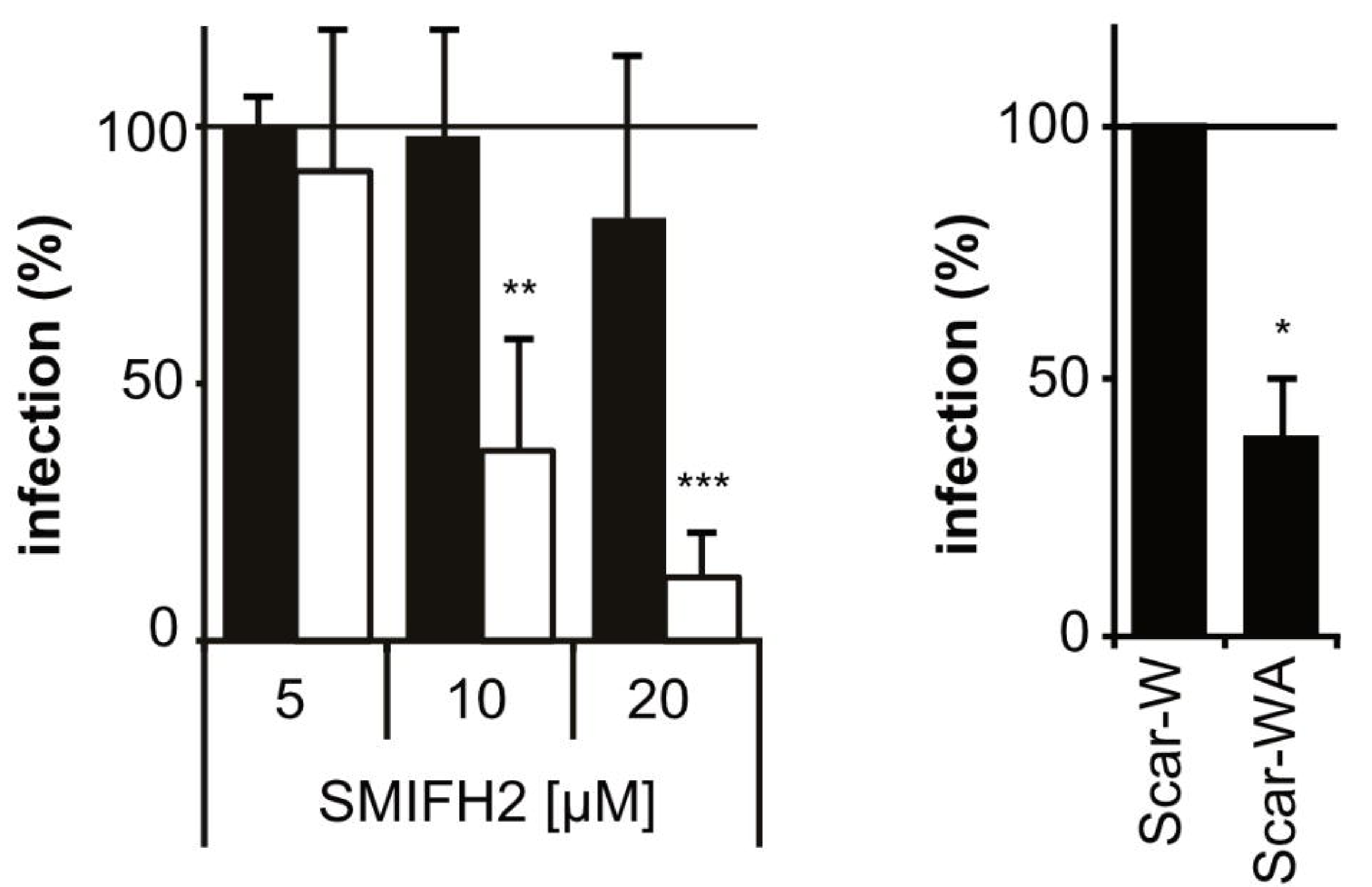

C

Arp3

घHPV16 口VV ISFV

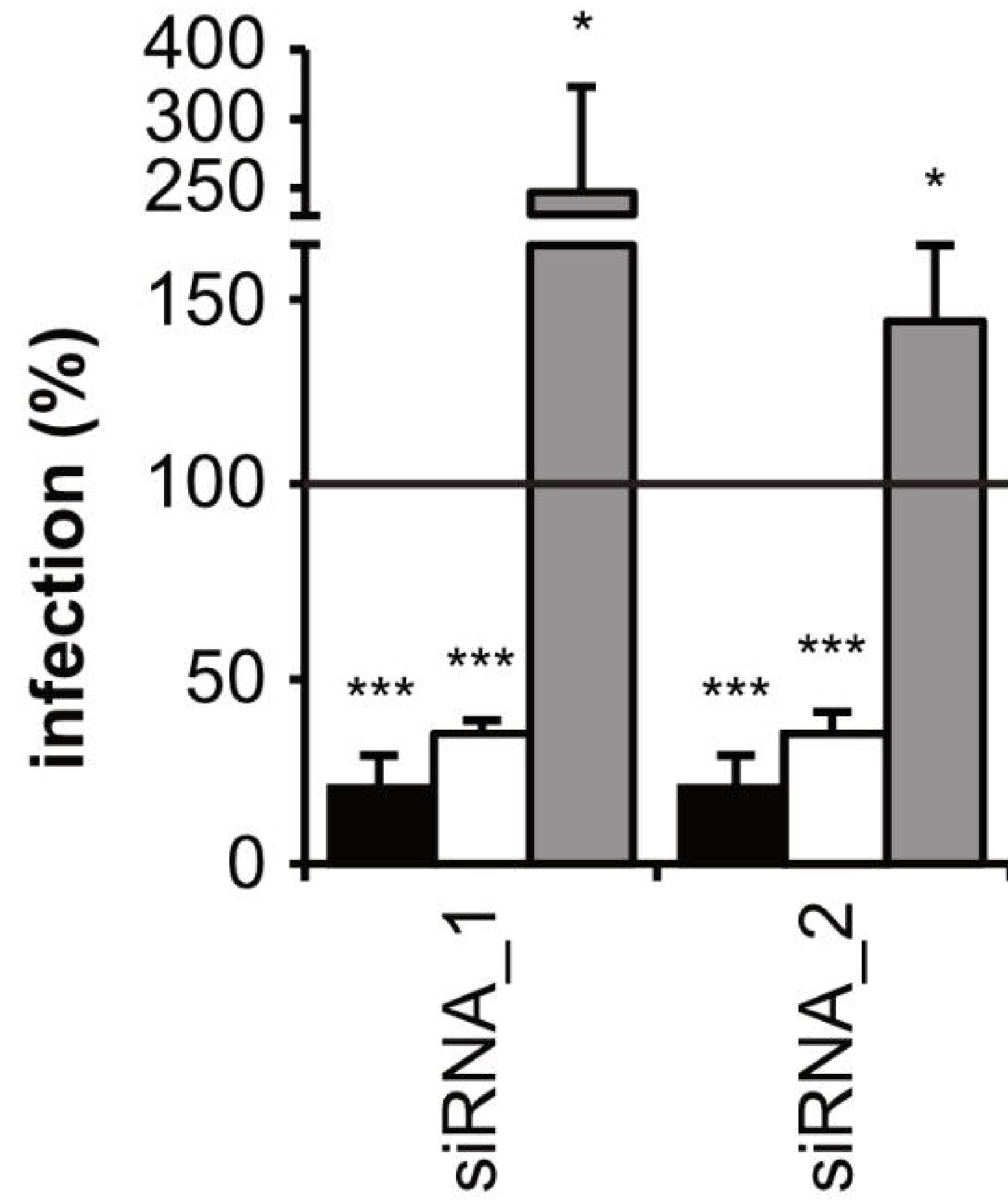

D

HPV16-pHrodo
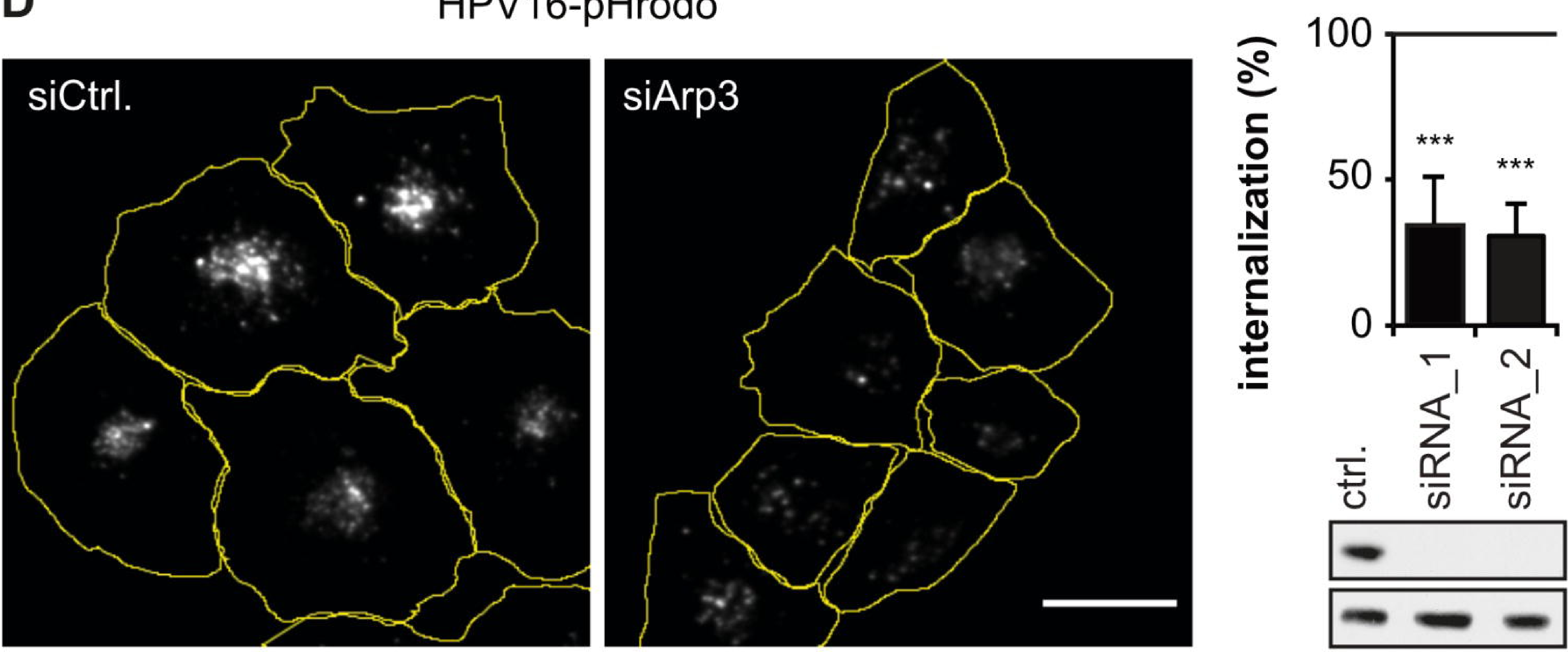

Arp3

tubulin 
A WASH RNAi

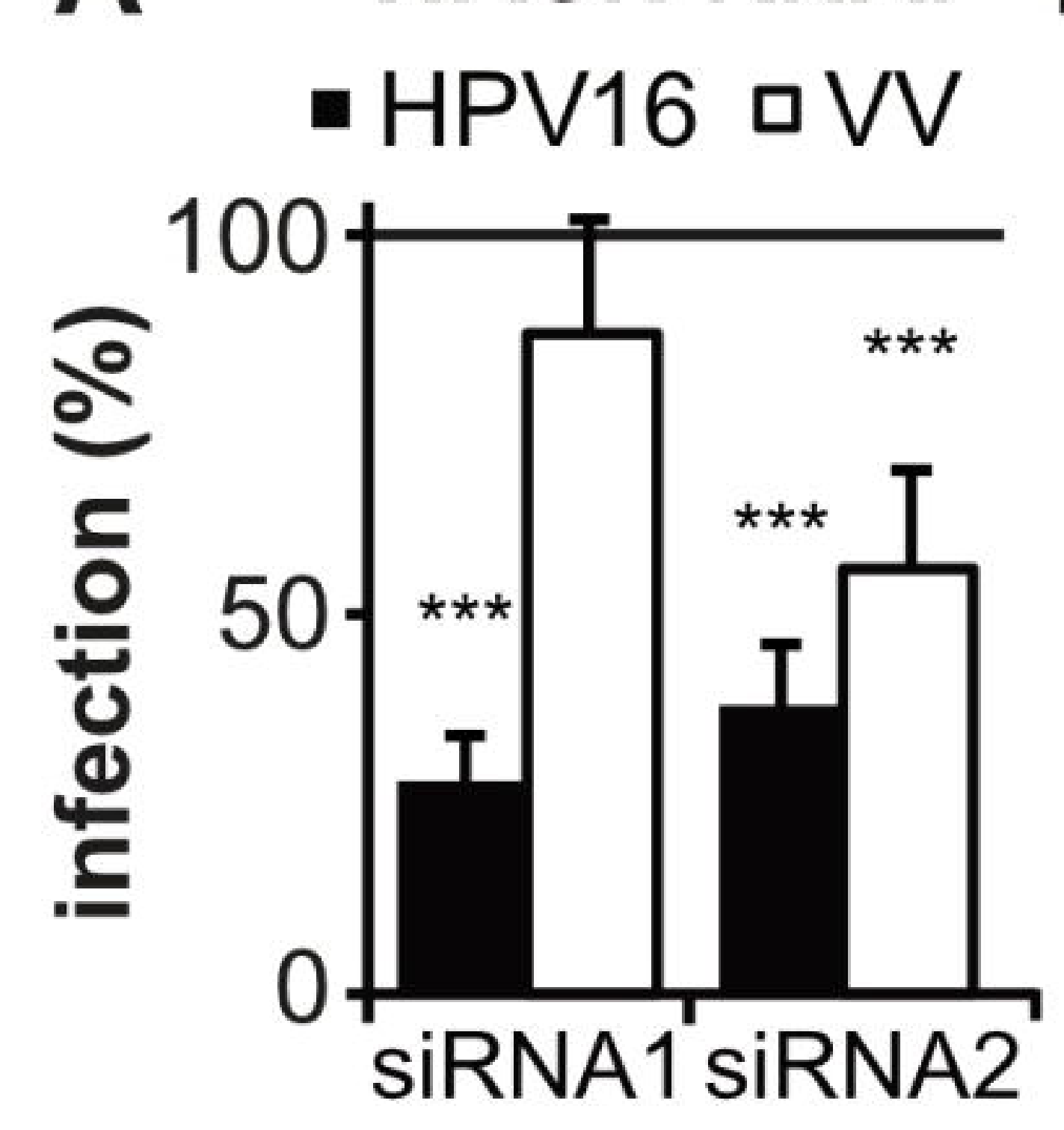

C WASH KO

- wild type aWASH KO

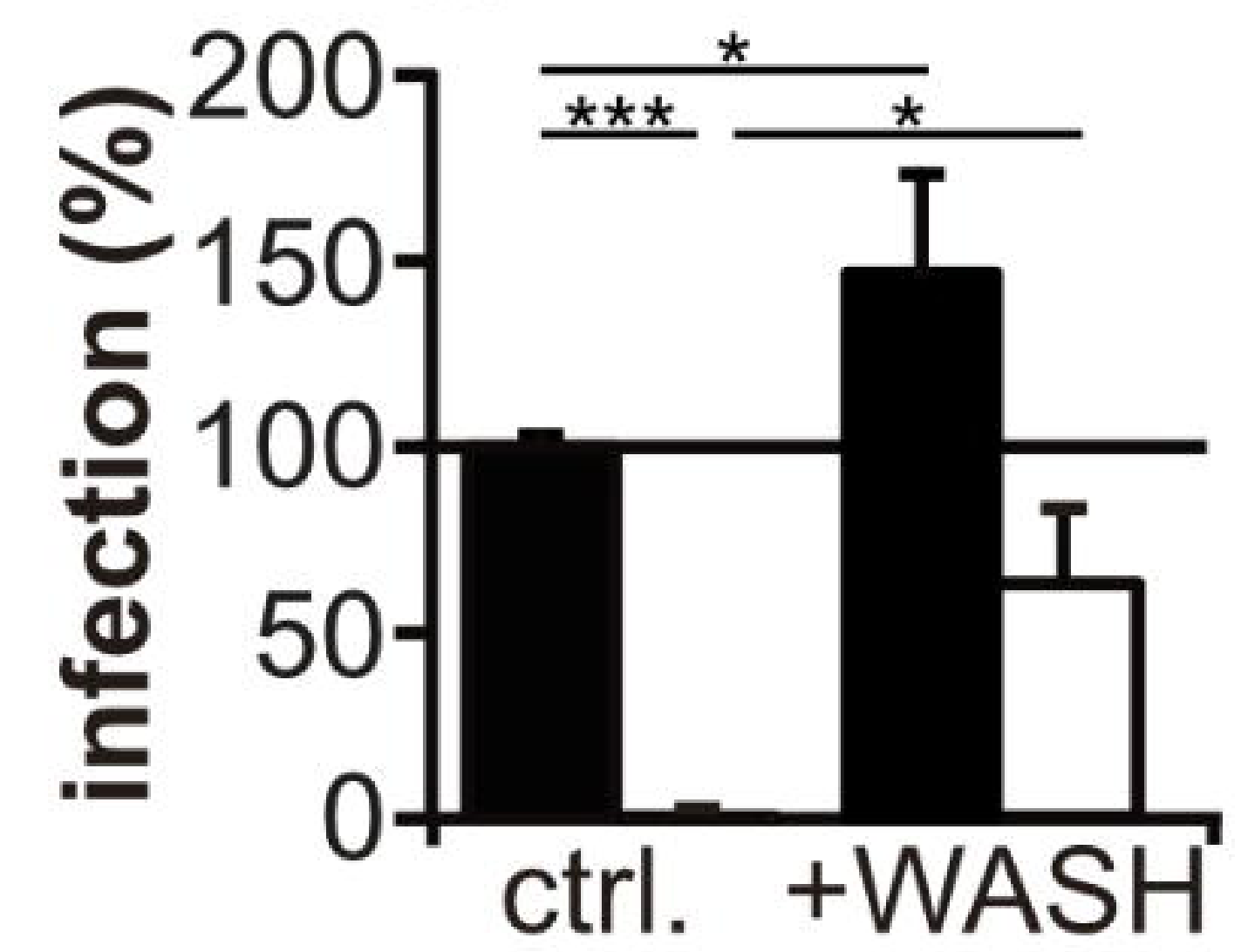

E
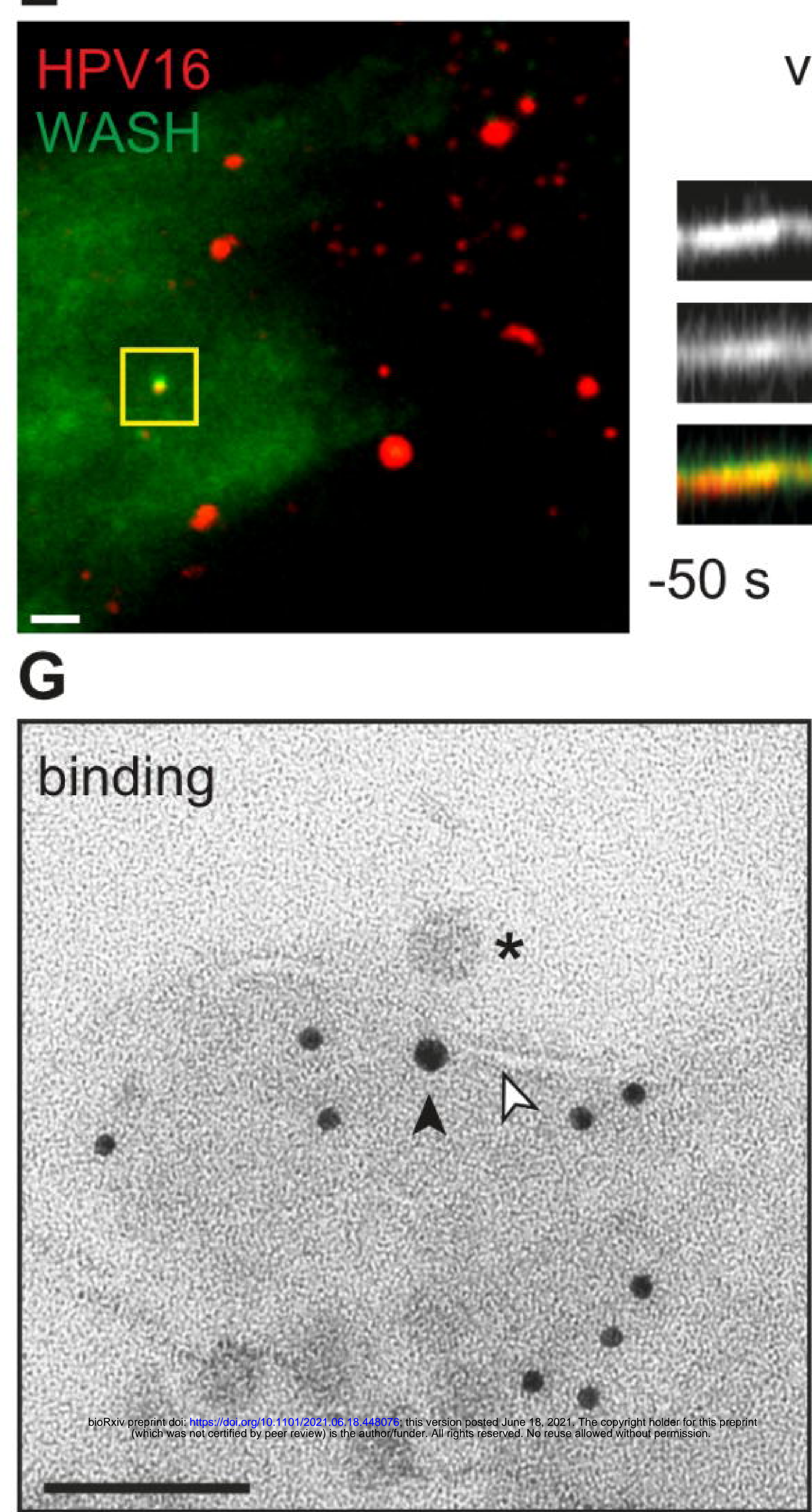

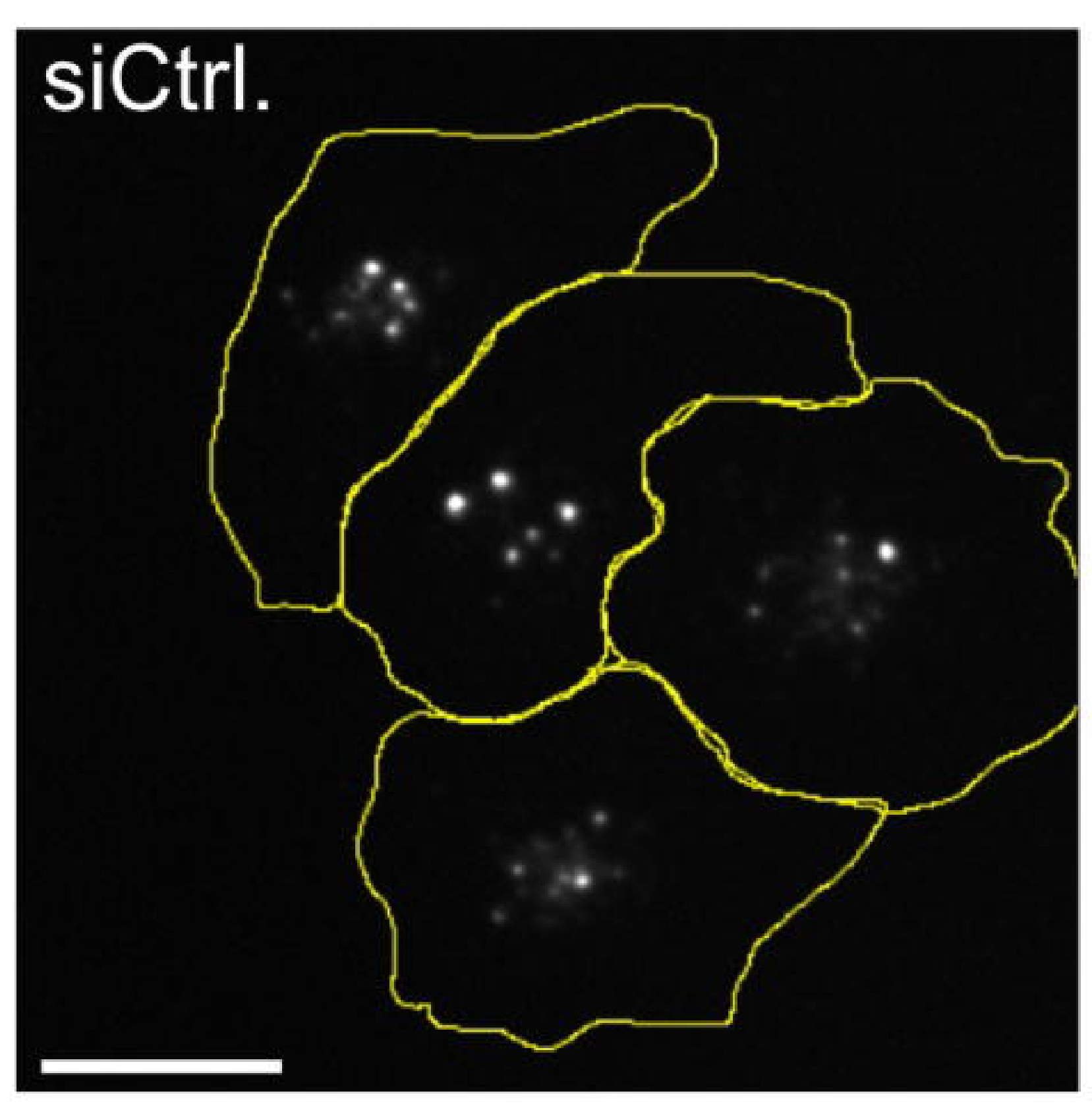

wild type
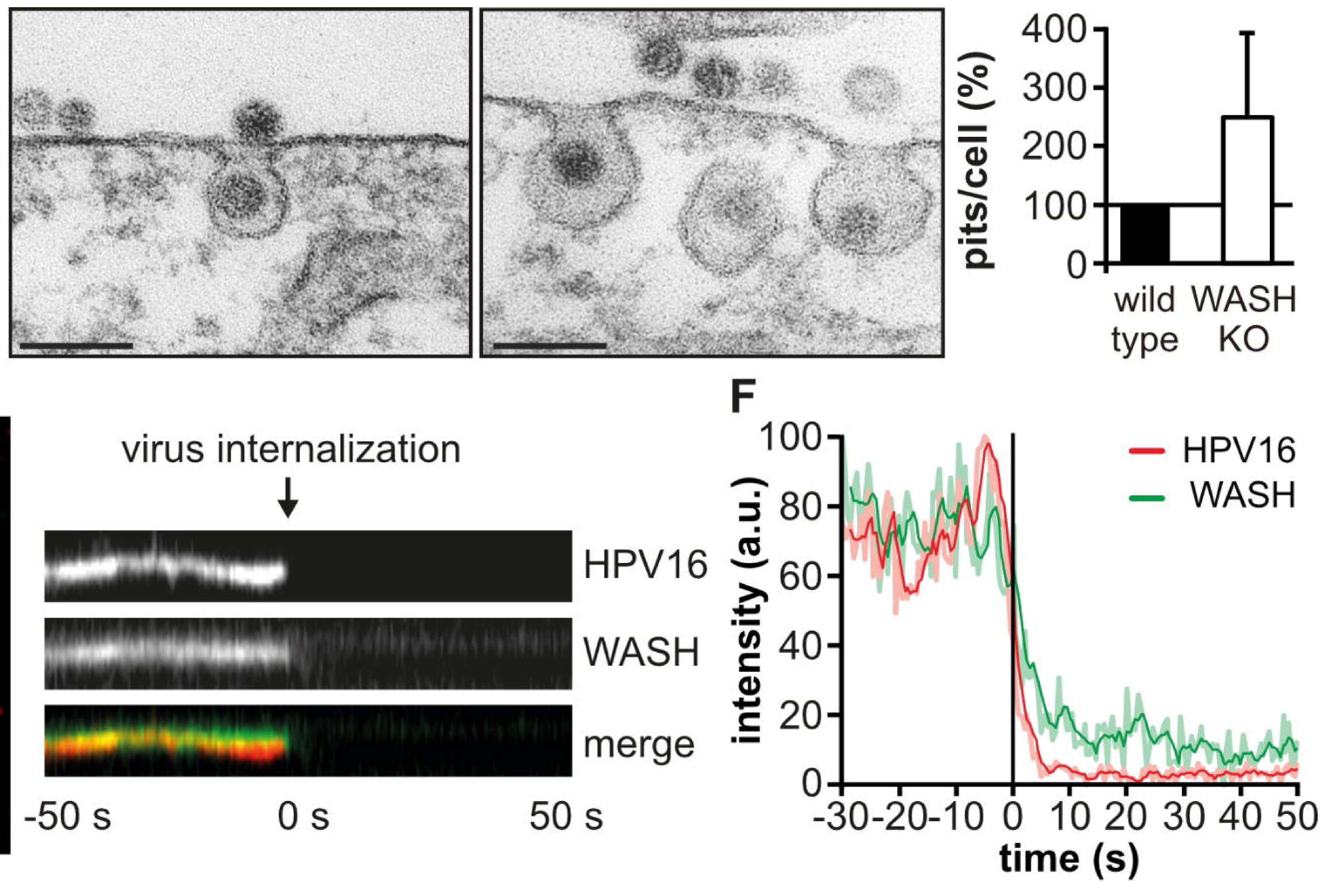

F

RNAi

- WASH aArp3

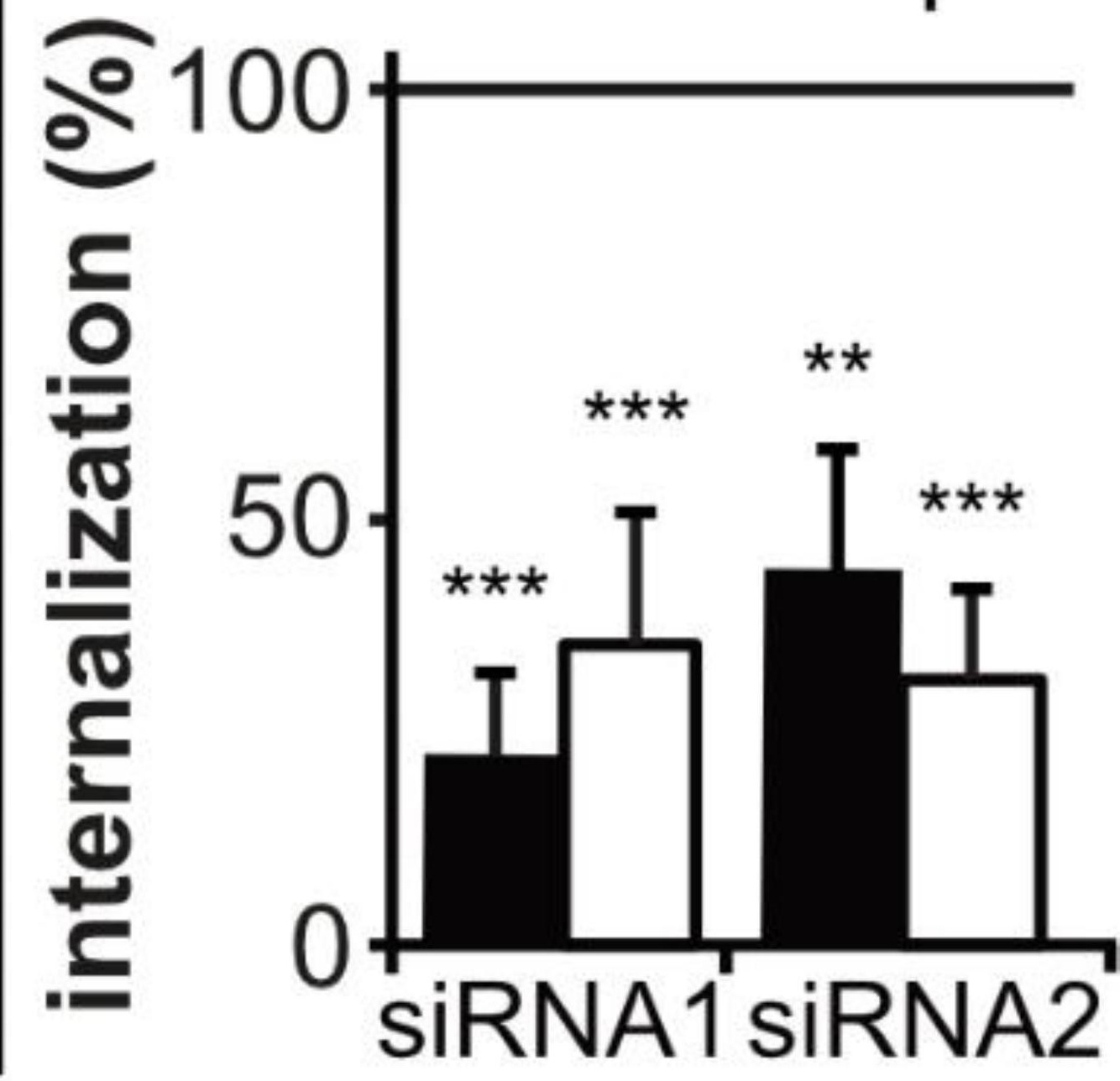

WASH KO

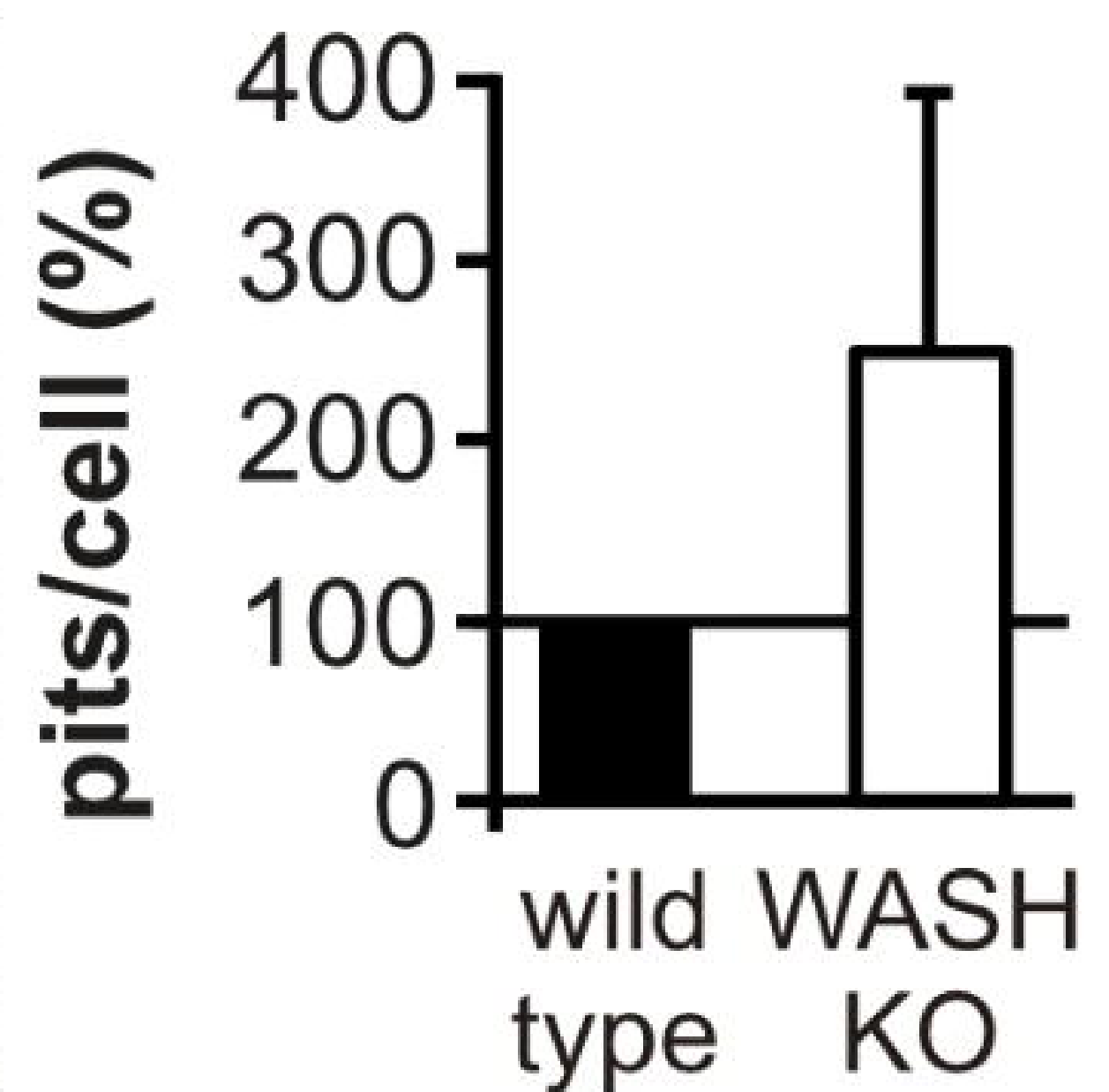

type KO

- HPV16

- WASH

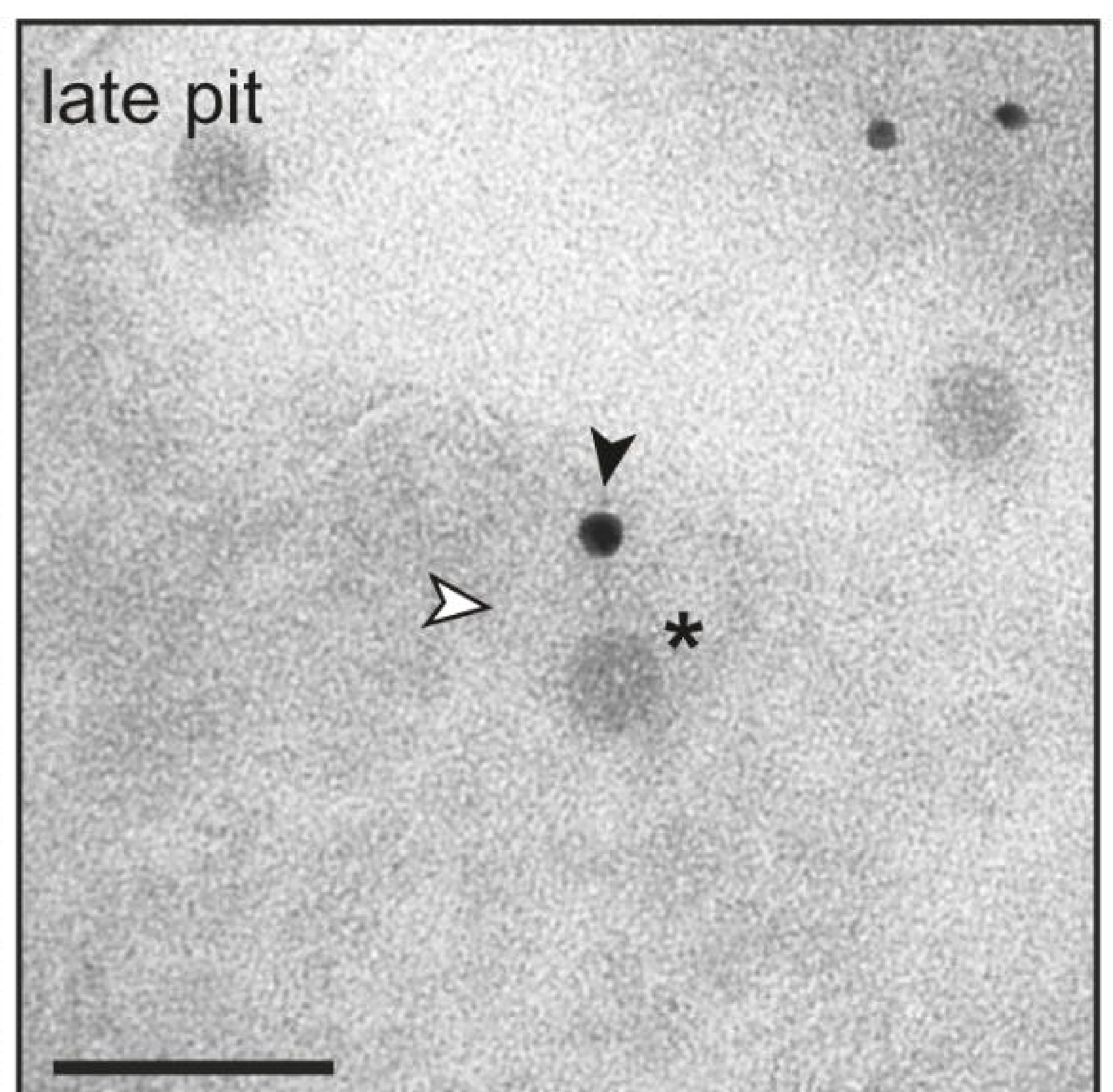



A $\square \mathrm{HPV} 16$ a HSV-1

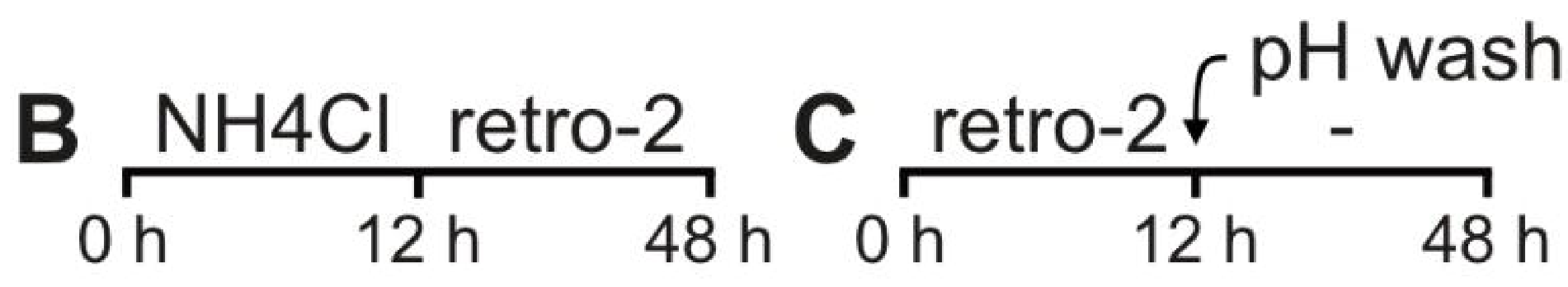
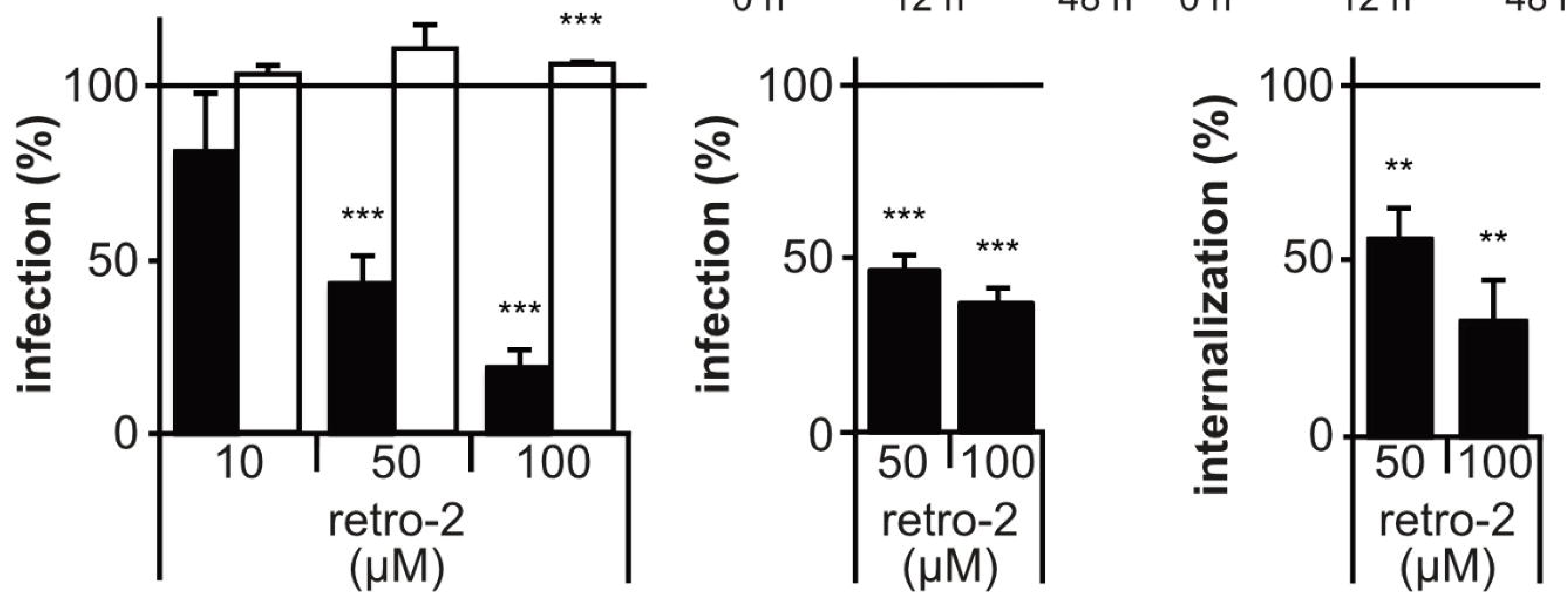

D
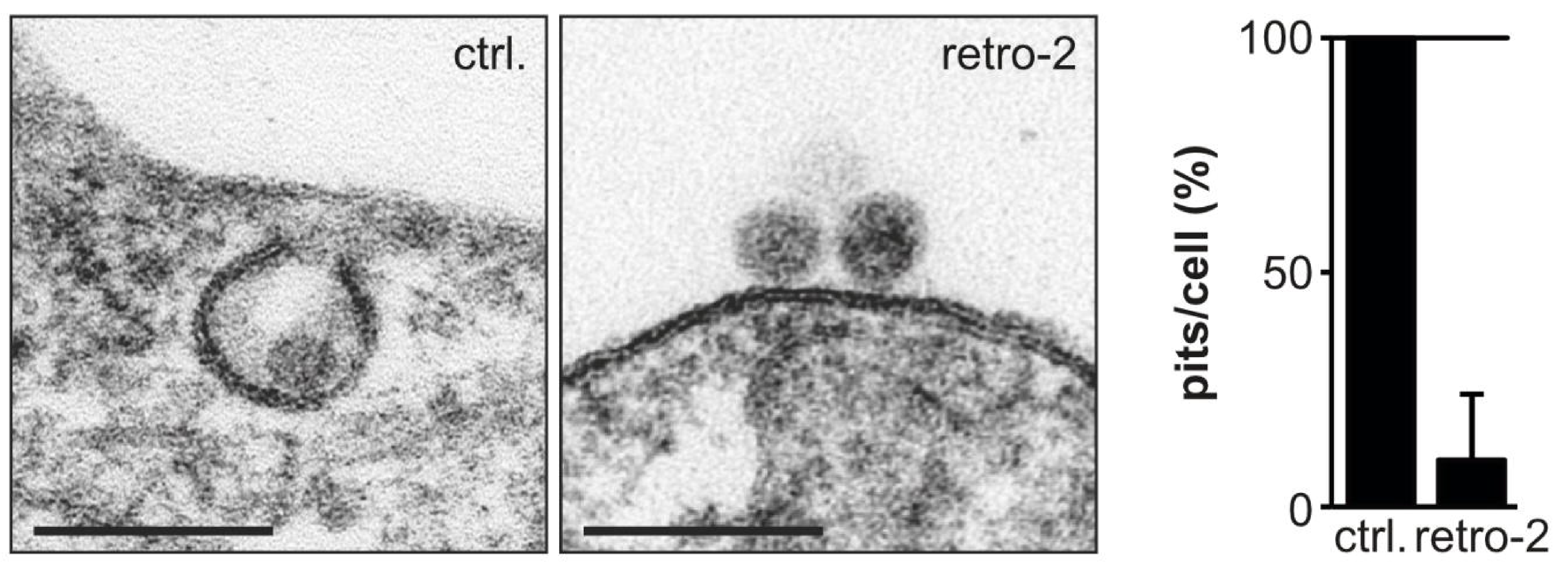
A
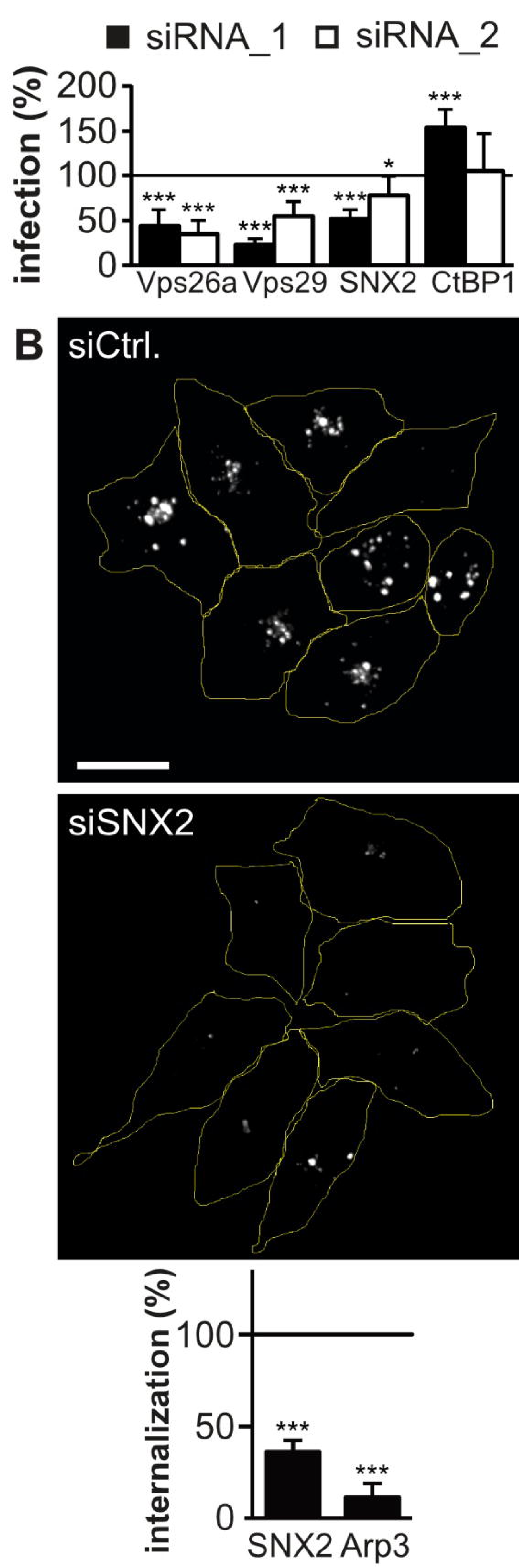

C

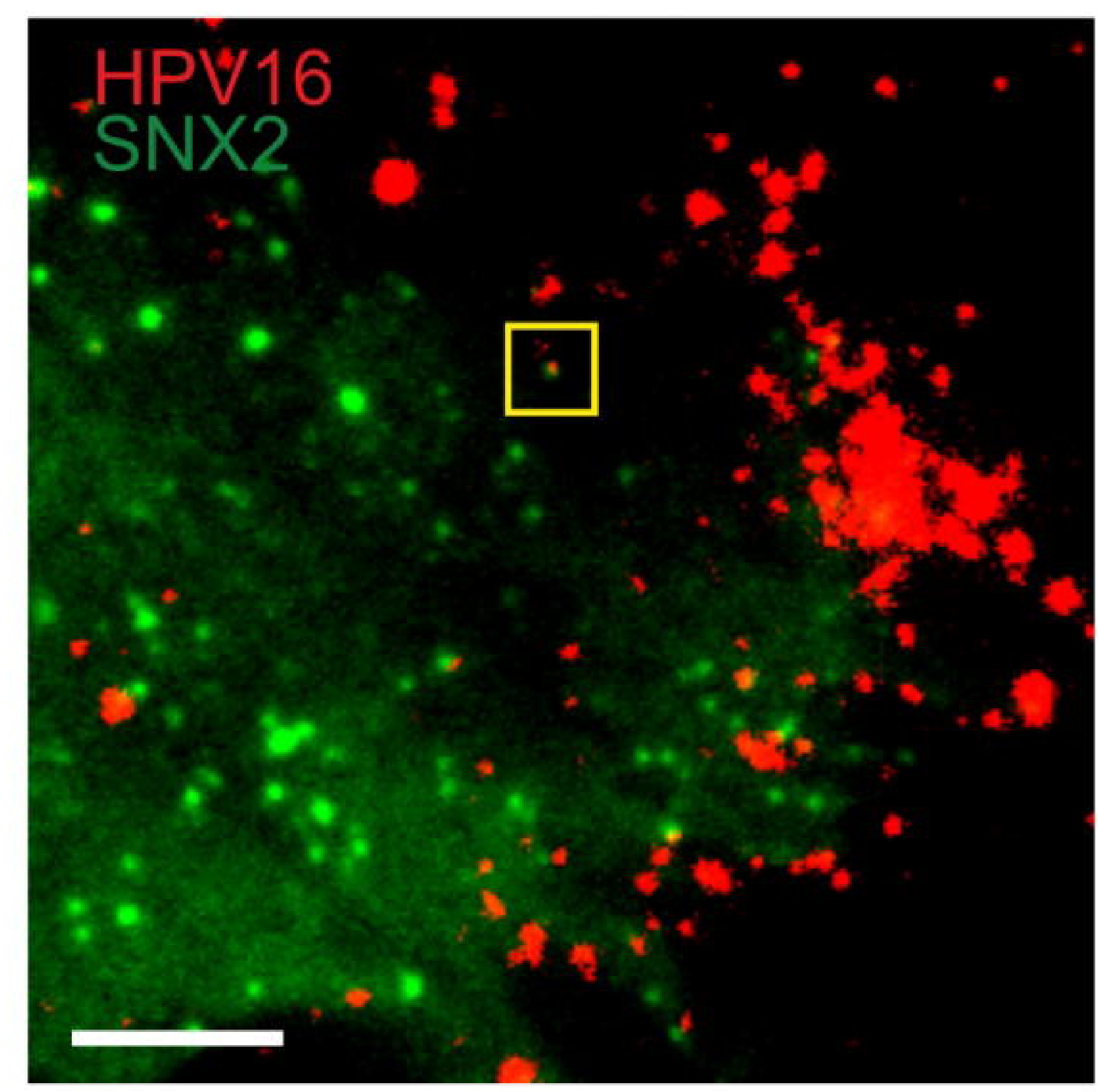

virus internalization

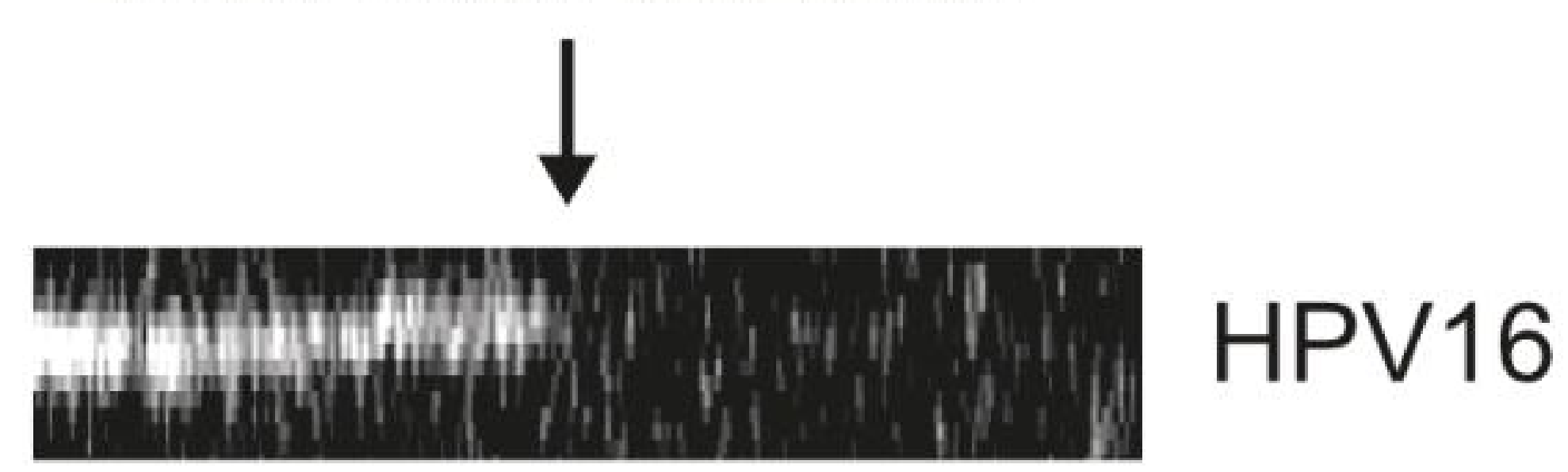

minementing
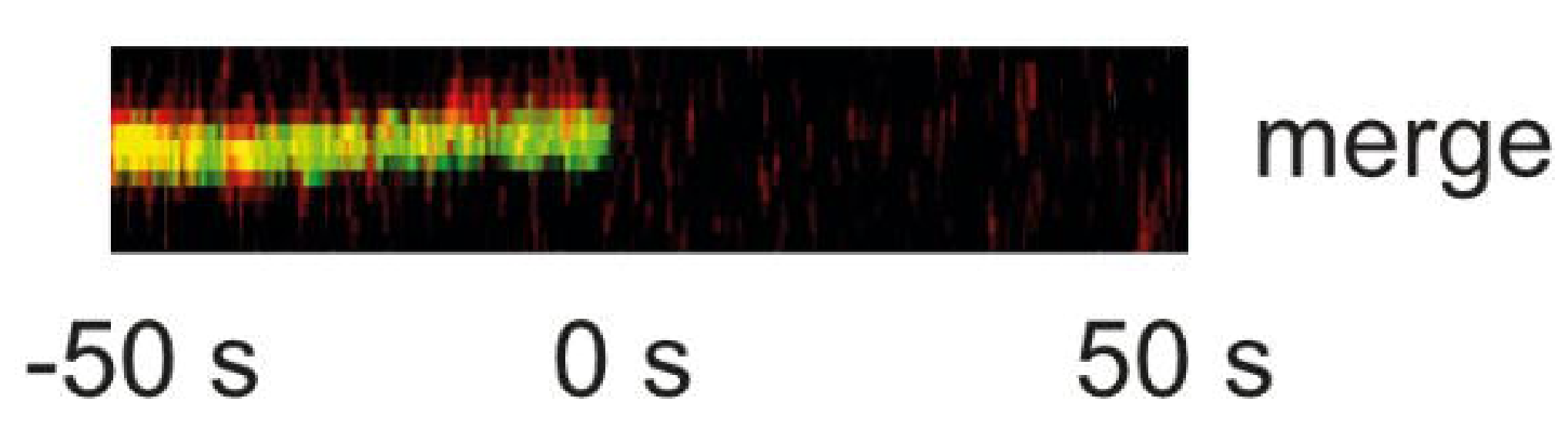

D

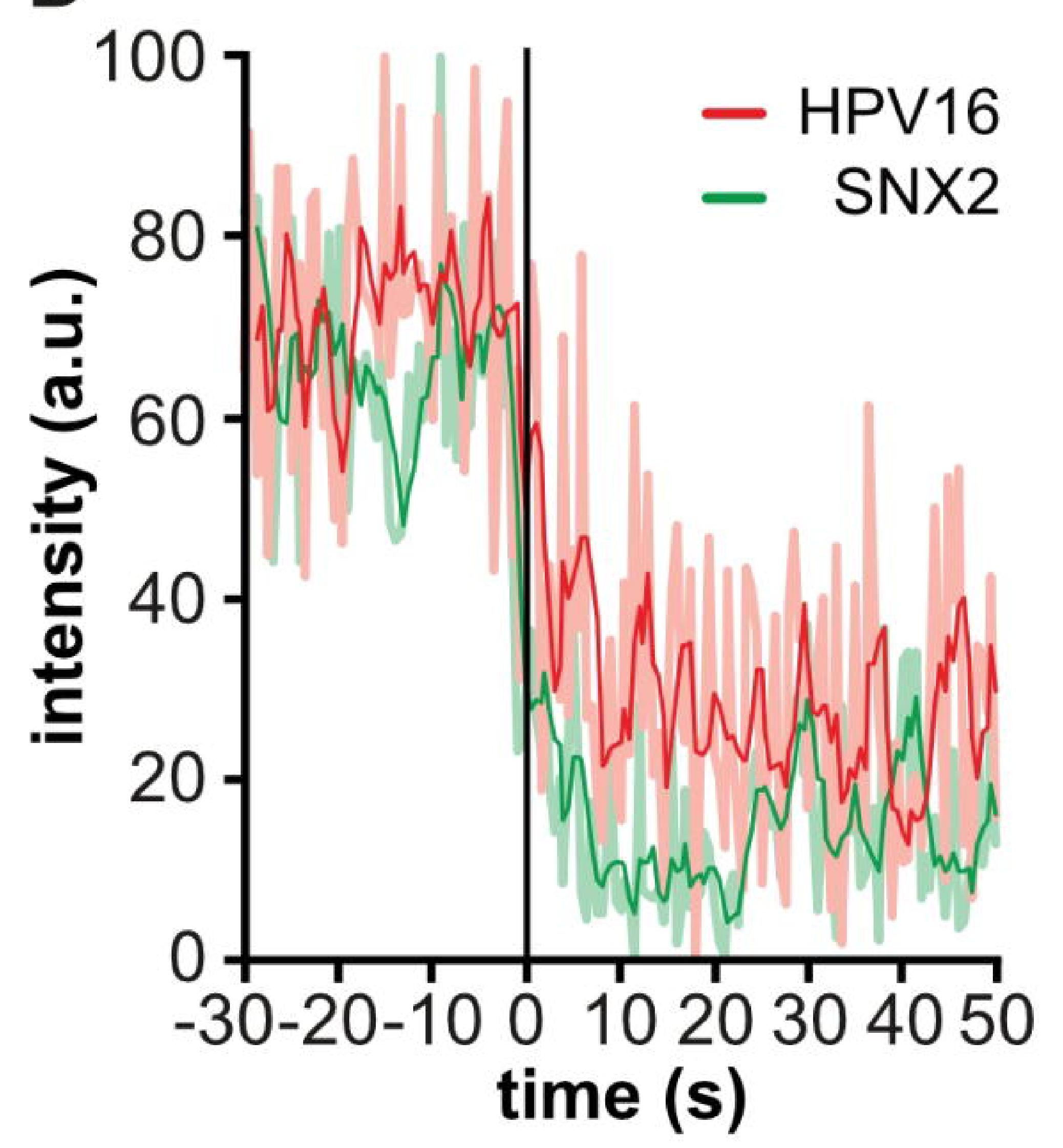

E

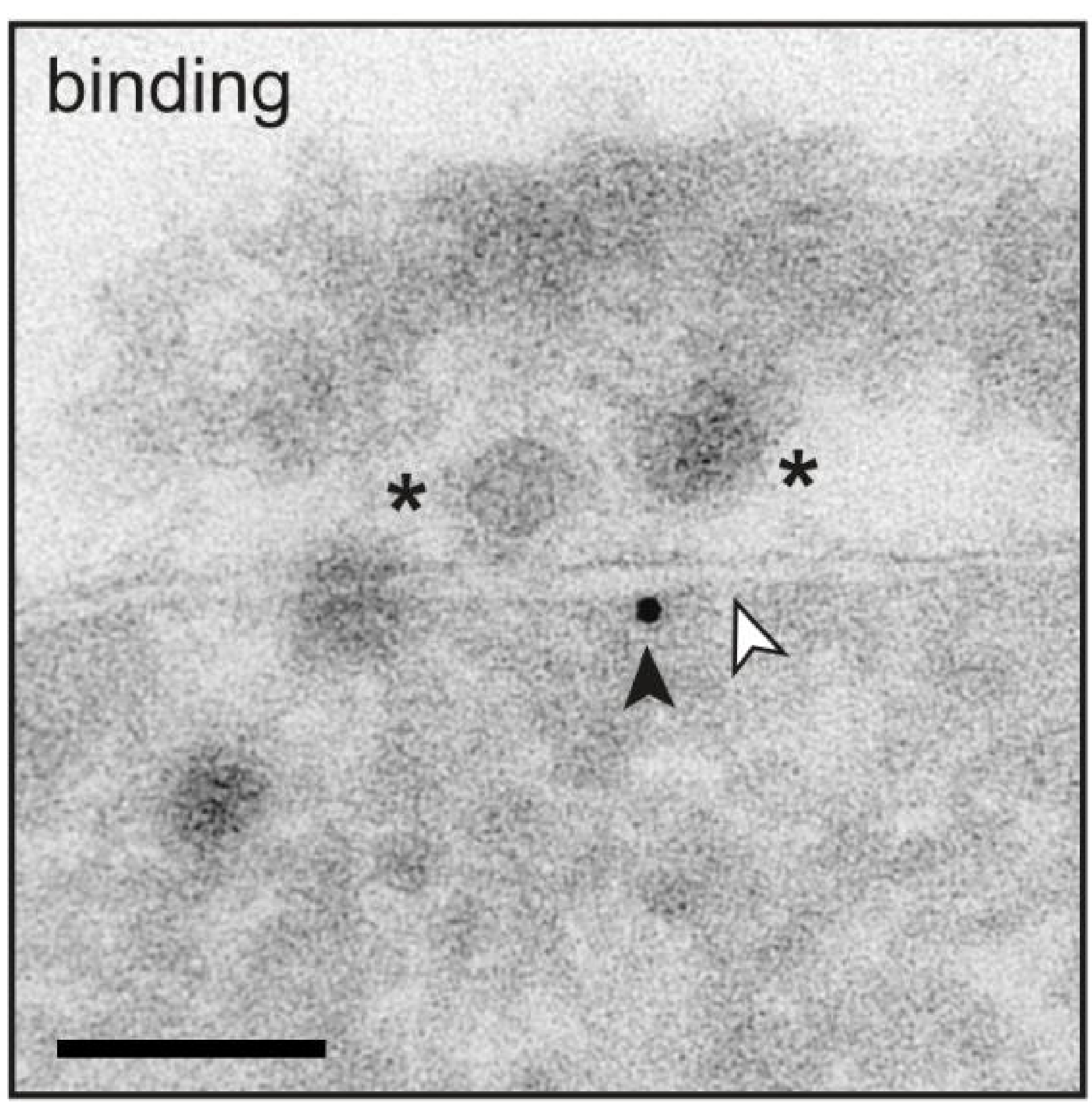

early pit
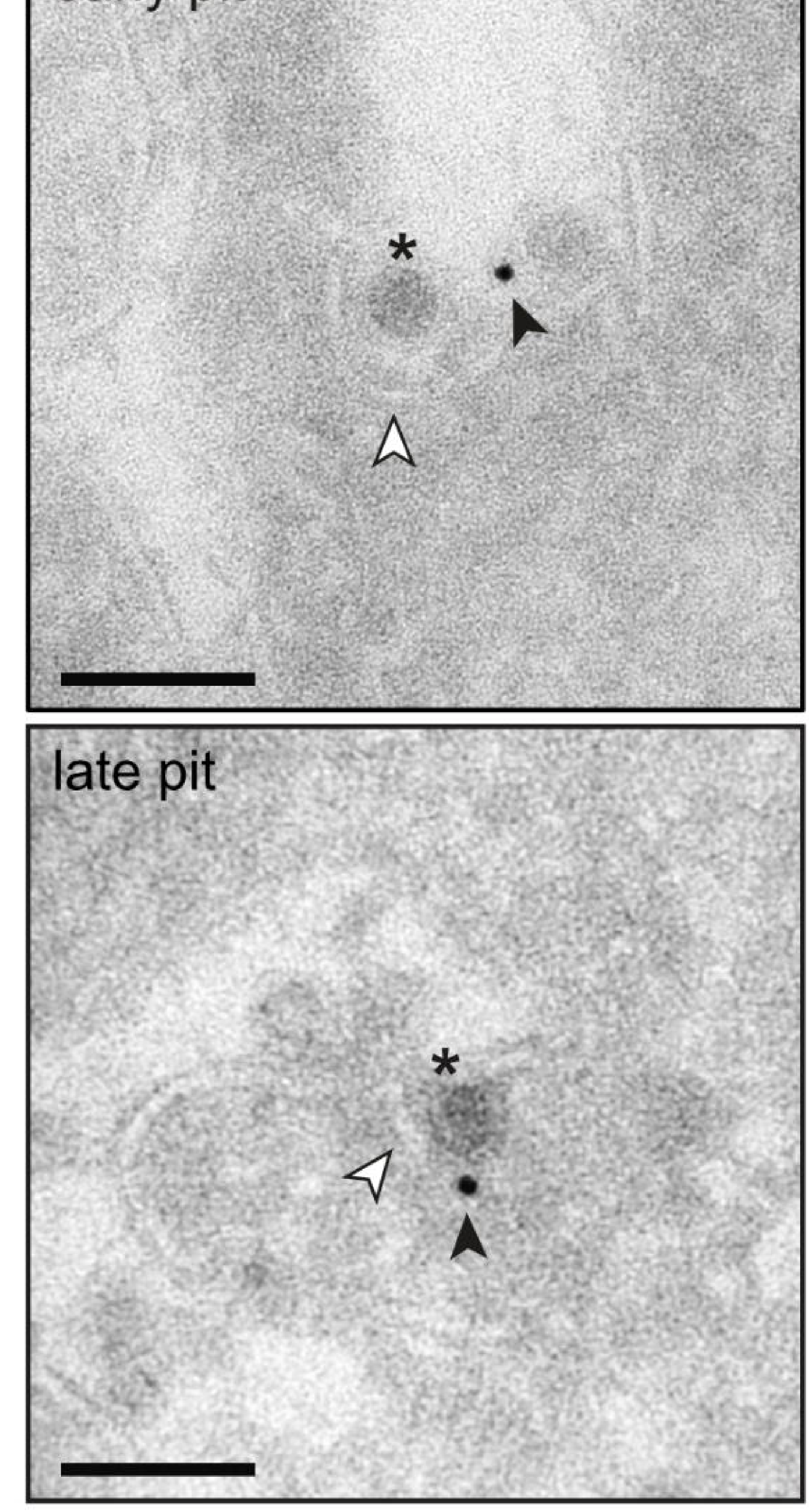


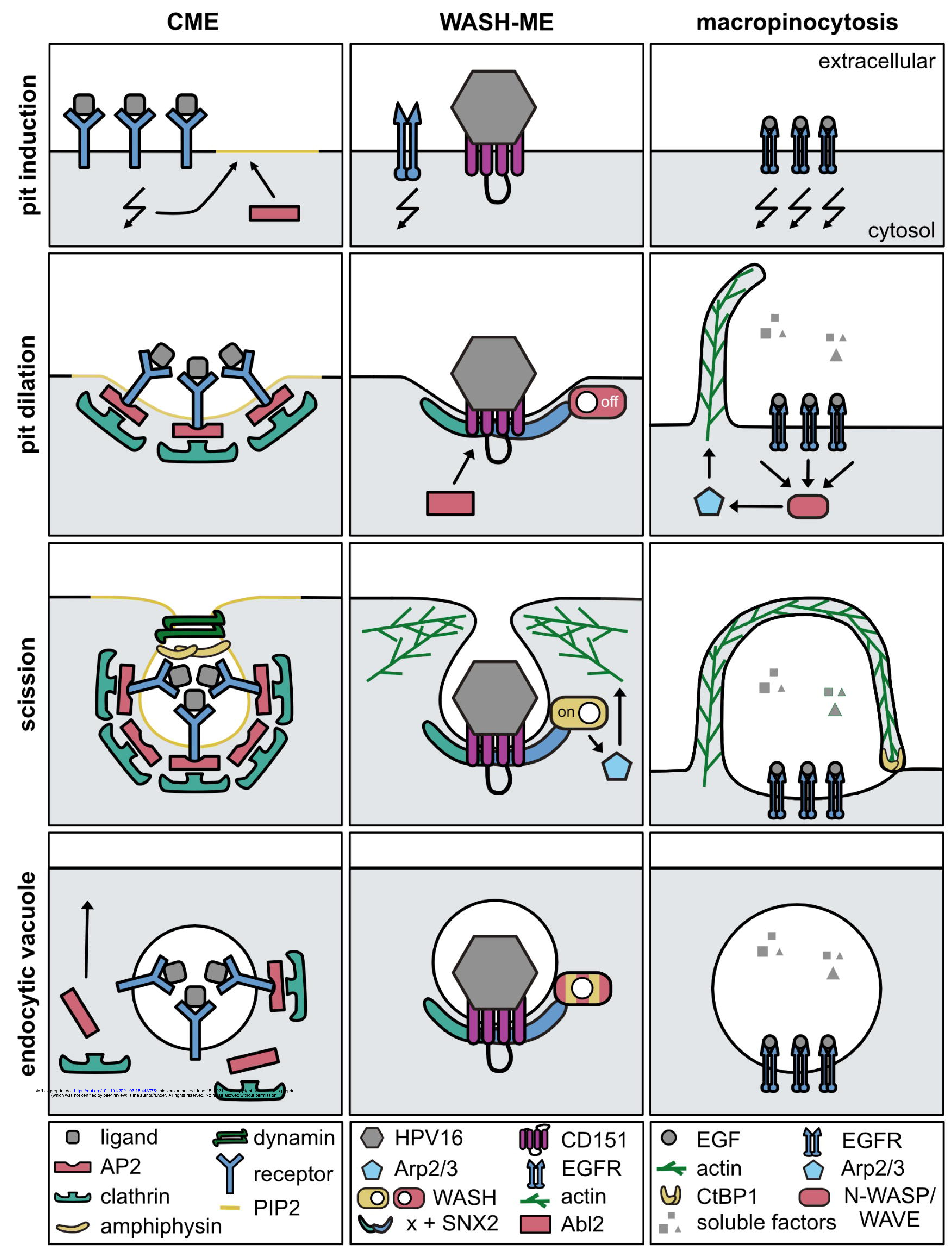

